\title{
On what I do not understand (and have something to say): Part I
}

\author{
by
}

Saharon Shelah (Jerusalem and New Brunswick, NJ)

\begin{abstract}
This is a non-standard paper, containing some problems in set theory I have in various degrees been interested in. Sometimes with a discussion on what I have to say; sometimes, of what makes them interesting to me, sometimes the problems are presented with a discussion of how I have tried to solve them, and sometimes with failed tries, anecdotes and opinions. So the discussion is quite personal, in other words, egocentric and somewhat accidental. As we discuss many problems, history and side references are erratic, usually kept to a minimum ("see ..." means: see the references there and possibly the paper itself).

The base were lectures in Rutgers, Fall '97, and reflect my knowledge then. The other half, [122], concentrating on model theory, will subsequently appear. I thank Andreas Blass and Andrzej Rosłanowski for many helpful comments.
\end{abstract}

\section{Contents}

1. Cardinal problems and pcf

2. The quest for the test: on the theory of Iterated Forcing for the continuum

3. Case studies for iterated forcing for the reals

4. Nicely defined forcing notions

5. To prove or to force, this is the question

6. Boolean algebras and iterated forcing

7. A taste of algebra

8. Partitions and colourings

9. Except forcing

10. Recent advances/comments

References

2000 Mathematics Subject Classification: Primary 03Exx.

Key words and phrases: set theory, cardinal arithmetic, pcf theory, forcing, iterated forcing, large continuum, nep, nicely definable forcing, combinatorial set theory, Boolean algebras, set-theoretic algebra, partition calculus, Ramsey theory.

I would like to thank Alice Leonhardt for the beautiful typing. Publication 666. Based on lectures in the Rutgers Seminar, Fall 1997 are: $\S 1, \S 2, \S 5, \S 7, \S 8$. 
1. Cardinal problems and pcf. Here, we deal with cardinal arithmetic as I understand it (see [161] or [166]), maybe better called cofinality arithmetic (see definitions below). What should be our questions? Wrong questions usually have no interesting answers or none at all.

Probably the most popular $\left({ }^{1}\right)$ question is a version (1.4 below) of:

Problem 1.1. Is $\operatorname{pp}\left(\aleph_{\omega}\right)<\aleph_{\omega_{1}}$ ?

Recall:

Definition 1.2. Let $\mathfrak{a}$ be a set of regular cardinals (usually $|\mathfrak{a}|<$ $\min (\mathfrak{a}))$. We define:

(1) $\operatorname{pcf}(\mathfrak{a})=\left\{\operatorname{cf}\left(\prod \mathfrak{a} / D\right): D\right.$ is an ultrafilter on $\left.\mathfrak{a}\right\}$.

(2) $\operatorname{cf}\left(\prod \mathfrak{a}\right)=\min \left\{|F|: F \subseteq \prod \mathfrak{a}\right.$ and $\left.\left(\forall g \in \prod \mathfrak{a}\right)(\exists f \in F)(g \leq f)\right\}$.

(3) For a filter $D$ on $\mathfrak{a}, \operatorname{tcf}\left(\prod \mathfrak{a} / D\right)=\lambda$ means that in $\prod \mathfrak{a} / D$ there is an increasing cofinal sequence of length $\lambda$.

(4) For a singular cardinal $\mu$ and a cardinal $\theta$ such that $\operatorname{cf}(\mu) \leq \theta<\mu$ let

$$
\begin{array}{r}
\operatorname{pp}_{\theta}(\mu)=\sup \left\{\operatorname{tcf}\left(\prod \mathfrak{a} / I\right): \mathfrak{a} \subseteq \operatorname{Reg} \cap \mu,|\mathfrak{a}|<\min (\mathfrak{a}), \sup (\mathfrak{a})=\mu,\right. \\
\left.I \text { an ideal on } \mathfrak{a} \text { such that } J_{\mathfrak{a}}^{\text {bd }} \subseteq I, \text { and }|\mathfrak{a}| \leq \theta\right\},
\end{array}
$$

where for a set $A$ of ordinals with no last element, $J_{A}^{\text {bd }}$ is the ideal of bounded subsets of $A$.

(5) Let $\operatorname{pp}(\mu)=\operatorname{pp}_{\operatorname{cf}(\mu)}(\mu)$.

(6) We define similarly $\operatorname{pp}_{\Gamma}(\mu)$ for a family (equivalently: a property) $\Gamma$ of ideals; e.g., $\Gamma(\theta, \tau)=$ the family of $(<\tau)$-complete ideals on a cardinal $<\theta, \Gamma(\theta)=\Gamma\left(\theta^{+}, \theta\right)$.

Definition 1.3. (1) For a partial order $P$,

$$
\operatorname{cf}(P)=\min \{|Q|: Q \subseteq P \text { and }(\forall p \in P)(\exists q \in Q)(p \leq q)\} .
$$

(2) For cardinals $\lambda, \mu, \theta, \sigma$,

$$
\begin{array}{r}
\operatorname{cov}(\lambda, \mu, \theta, \sigma)=\min \left\{|\mathcal{A}|: \mathcal{A} \subseteq[\lambda]^{<\mu} \text { and any } a \in[\lambda]^{<\theta}\right. \text { is included } \\
\text { in the union of }<\sigma \text { members of } \mathcal{A}\} .
\end{array}
$$

Problem 1.1 is for me the right form of

QUestion 1.4. (1) Assume $\aleph_{\omega}$ is strong limit. Is $2^{\aleph_{\omega}}<\aleph_{\omega_{1}}$ ?

(2) Assume $2^{\aleph_{0}}<\aleph_{\omega}$. Is $\left(\aleph_{\omega}\right)^{\aleph_{0}}<\aleph_{\omega_{1}}$ ?

Why do I think 1.1 is a better form? Because we know that:

$(*)_{1} \quad$ If $\aleph_{\omega}$ is strong limit, then $2^{\aleph_{\omega}}=\left(\aleph_{\omega}\right)^{\aleph_{0}}$ (classical cardinal arithmetic).

$\left(^{1}\right)$ That is, most people who are aware of this direction will mention it, and probably many have tried it to some extent. 
$(*)_{2} \quad \operatorname{pp}\left(\aleph_{\omega}\right)=\operatorname{cf}\left(\left[\aleph_{\omega}\right]^{\aleph_{0}}, \subseteq\right)($ see $[98])$,

$(*)_{3} \quad \aleph_{\omega}^{\aleph_{0}}=2^{\aleph_{0}}+\operatorname{cf}\left(\left[\aleph_{\omega}\right]^{\aleph_{0}}, \subseteq\right)$ (trivial).

So the three versions are equivalent and say the same thing when they say something at all, but Problem 1.1 is always meaningful.

To present what I think are central problems, we can start from what I called the solution of "Hilbert's first problem", see [178] (though without being seconded).

THEOREM 1.5. For $\lambda \geq \beth_{\omega}$, there are $\kappa<\beth_{\omega}$ and $\mathcal{P} \subseteq[\lambda]^{<\beth_{\omega}}$ with $|\mathcal{P}|=\lambda$ such that every $A \in[\lambda]^{<\beth_{\omega}}$ is equal to the union of $<\kappa$ members of $\mathcal{P}$.

So $\mathcal{P}$ is "very dense". E.g., if $c:[\lambda]^{n} \rightarrow \beth_{n}$ then for some $B_{m} \in \mathcal{P}$ (for $m<\omega$ ), the restrictions $c \uparrow\left[B_{m}\right]^{n}$ are constant and $\left|B_{m}\right|=\beth_{m}$. We can replace $\beth_{\omega}$ by any strong limit cardinal $>\aleph_{0}$.

In $[103, \S 8]$ the following application of 1.5 to the theory of Boolean algebras is proved:

THEOREM 1.6. If $B$ is a c.c.c. Boolean algebra and $\mu=\mu^{\beth_{\omega}} \leq|B| \leq 2^{\mu}$, then $B$ is $\mu$-linked, i.e., $B \backslash\{0\}$ is the union of $\mu$ sets of pairwise compatible elements.

(See also [139], [141] and Hajnal, Juhász and Szentmiklóssy [55].)

We also have the following application:

TheOREM 1.7 (see [167]). If $X$ is a topological space (not necessarily $T_{2}$ ) with $\lambda$ points, $\mu \leq \lambda<2^{\mu}$, and with $>\lambda$ open sets, and $\mu$ is strong limit of cofinality $\aleph_{0}$, then $X$ has $\geq 2^{\mu}$ open sets.

Another connection to the general topology is the following

Definition 1.8. For topological spaces $X, Y$ and a cardinal $\theta$, write $X \rightarrow(Y)_{\theta}^{1}$ iff for every partition $\left\langle X_{i}: i<\theta\right\rangle$ of $X$ into $\theta$ parts, $X$ has a closed subspace $Y^{\prime}$ homeomorphic to $Y$ which is included in one part of the partition.

A quite well known problem (we shall call it TCFC, the topological discontinua free coloring) asked whether for every compact Hausdorff space $X$,

$$
X \nrightarrow(\text { Cantor discontinuum })_{2}^{1} \text {. }
$$

Now TCFC problem $+\neg \mathrm{CH}$ is sandwiched between two pcf statements of which we really do not know whether they are true (so in particular implies the existence of inner models with quite large cardinals). If for simplicity $2^{\aleph_{0}} \geq \aleph_{3}$, then e.g.: 
$(*)_{1} \quad$ If for no $\mathfrak{a} \subseteq \operatorname{Reg}$, where Reg is the class of regular cardinals, $|\mathfrak{a}| \geq$ $\aleph_{2}$ and $\prod \mathfrak{a} /[\mathfrak{a}] \leq \aleph_{0}$ is $\sup (\mathfrak{a})$-directed, then the answer is: for every Hausdorff space $X$, we have $X \nrightarrow$ (Cantor discontinuum) ${ }_{2}^{1}$ and more.

$(*)_{2} \quad$ If for some $\mathfrak{a} \subseteq \operatorname{Reg} \backslash 2^{<\kappa},|\mathfrak{a}|=2^{\aleph_{0}} \leq \kappa$ and $\prod \mathfrak{a} /[\mathfrak{a}] \leq \aleph_{0}$ is $\sup (\mathfrak{a})$ directed, then in some forcing extension there exists a zero-dimensional Hausdorff space $X$ such that $X \rightarrow$ (Cantor discontinuum $)_{2}^{1}$. The Stone-Cech compactification of this space gives a negative answer to the TCFC question.

(On the problem, see [178] and more in [99]; in particular under CH starting with supercompact "yes" is consistent and still we need large cardinals.)

However, we can start from inside pcf theory.

Problem 1.9. Is $\operatorname{pcf}(\mathfrak{a})$ countable for each countable set of cardinals?

This seems to me more basic than 1.1, yet 1.1 is weaker. I think it is better to look at the battlefield between independence by forcing from large cardinals and proofs in ZFC (I would tend to say between the armies of Satan and God but the armies are not disjoint).

The advances in pcf theory show us ZFC is more powerful than expected before. I will try to give a line of statements on which both known methods fail - so far.

Conjecture 1.10. If $\mathfrak{a}$ is a set of regular cardinals $>|\mathfrak{a}|$, then for no inaccessible $\lambda$ is the intersection $\lambda \cap \operatorname{pcf}(\mathfrak{a})$ unbounded in $\lambda$.

Conjecture 1.11. For every $\mu \geq \aleph_{\omega}$, for every $\aleph_{n}<\aleph_{\omega}$ large enough there is no $\lambda<\mu$ of cofinality $\aleph_{n}$ such that $\operatorname{pp}_{\Gamma\left(\aleph_{n}\right)}(\lambda)>\mu$ (or replace $\aleph_{n}<\aleph_{\omega}$ by $\aleph_{\alpha}<\aleph_{\omega^{2}}$ or even $\aleph_{\alpha}<\aleph_{\omega_{1}}$, or whatever).

Conjecture 1.12. (A) It is consistent, for any uncountable $\theta$ (e.g., $\aleph_{1}$ ), that for some $\lambda$,

$$
\theta \leq\left|\left\{\mu<\lambda: \operatorname{cf}(\mu)=\aleph_{0}, \operatorname{pp}(\mu)>\lambda\right\}\right| .
$$

(B) It is consistent that for some $\lambda$, the set

$$
\left\{\mu<\lambda: \operatorname{cf}(\mu)>\aleph_{0}, \operatorname{pp}_{\aleph_{1}-\text { complete }}(\mu)>\lambda\right\}
$$

is infinite.

Those three conjectures seem to be fundamental. Note that having ZFCprovable answer in 1.10,1.11, but independent answer for 1.12 are conscious choices. For all of those problems, present methods of independence fail, and in addition they are known to require higher consistency strength. Of course, we can concentrate on other variants; e.g., in 1.12(B) use $\theta$ instead of $\aleph_{0}$.

Other problems tend to be sandwiched between those, or at least those more basic problems are embedded into them. E.g., 1.11 implies that in 1.5 we can replace $\beth_{\omega}$ by $\aleph_{\omega}$ if we replace equal by included (or demand 
$\left.\lambda \geq \sum_{n<\omega} 2^{\aleph_{n}}\right)$ and this implies $|\mathfrak{a}| \leq \aleph_{0} \Rightarrow|\operatorname{pcf}(\mathfrak{a})| \leq \aleph_{\omega}$, while e.g. $|\mathfrak{a}| \leq$ $\aleph_{\omega n} \Rightarrow|\operatorname{pcf}(\mathfrak{a})| \leq \aleph_{\omega n+\omega}$ implies the analog of 1.5 for $\aleph_{\omega^{2}}$ (see [178], [123]). See [123] for more on the ZFC side; it may be very helpful in preventing futile attempts to force.

Note that $\operatorname{pp}\left(\aleph_{\omega}\right)>\aleph_{\omega_{1}}$ implies that for some countable $\mathfrak{a}, \operatorname{pcf}(\mathfrak{a})$ is uncountable, which implies that clause (A) of Conjecture 1.12 holds. Also $\operatorname{pp}\left(\aleph_{\omega}\right)>\aleph_{\omega_{2}}$ implies that $|\operatorname{pcf}(\mathfrak{a})| \geq \aleph_{2}$ for some countable $\mathfrak{a}$, which implies that clause (B) of Conjecture 1.12 fails.

So there is no point to try to prove $\operatorname{CON}\left(\operatorname{pp}\left(\aleph_{\omega}\right)>\aleph_{\omega_{1}}\right)$ before having the consistency of $1.12(\mathrm{~A})$ and, thus, $\operatorname{CON}\left(\operatorname{pp}\left(\aleph_{\omega}\right)>\aleph_{\omega_{1}}\right)$ is a more specialized case. (Also if we look at the earlier history of consistency proofs - clearly there is no point to start with Problem 1.1.)

In Conjecture 1.10 the situation (which we say is impossible) may look bizarre, as $\operatorname{pcf}(\mathfrak{a})$ is extremely large. Of course, much better is $|\operatorname{pcf}(\mathfrak{a})|<$ "first inaccessible $>|\mathfrak{a}|$ " and even $|\operatorname{pcf}(\mathfrak{a})| \leq|\mathfrak{a}|^{+\omega}$, which follows from Conjecture 1.11. Of course, replacing in 1.10, " $\lambda$ inaccessible" by " $\lambda$ Mahlo" is still a very important conjecture (to me) while getting $\operatorname{pcf}(\mathfrak{a})<$ "the first fixed point $>|\mathfrak{a}|$ " is much better, so why from all variants of 1.10 , those we have just mentioned and others, "the accumulation inaccessible" was chosen? The point is that it implies

(*) $\quad \operatorname{cf}\left(\prod \operatorname{pcf}(\mathfrak{a})\right)=\operatorname{cf}\left(\prod \mathfrak{a}\right) \quad$ if $\mathfrak{a}$ is a set of regular cardinals $>|\mathfrak{a}|$

(see [166, Ch. VIII, §3], [97]; note that in the notation of [97], conjecture 1.10 says that $\left.\operatorname{pcf}(\mathfrak{a}) \in J_{\mathfrak{a}}^{*}\right)$. If there is a failure of Conjecture 1.10 then consistently $(*)$ fails. We can force by $(<\lambda)$-complete forcing iterating adding $f \in \prod(\operatorname{pcf}(\mathfrak{a}) \cap \lambda)$ dominating the old product (or for any $\mu$, just adding $\mu$ many $\lambda$-Cohen functions, i.e., forcing with

$$
\{f: f \text { is a partial function from } \mu \text { to } \lambda,|\operatorname{Dom}(f)|<\lambda\}) .
$$

So 1.10 denotes a significant dividing line between chaos and order.

Concerning the last conjecture 1.12, maybe the proofs in Gitik and Shelah [45] are relevant. There we force for hypermeasurable cardinals $\kappa_{0}<\kappa_{1}<\ldots<\kappa_{n}$ with a forcing which makes each $\kappa_{i}$ hypermeasurable indestructible under reasonable forcing notions, including those which may add new Prikry sequences of ordinals $>\kappa_{l}$ of length $<\kappa_{l}$. (So in this case supercompact cannot serve, unlike in many proofs which do with hypermeasurable cardinals what is relatively easy to do with supercompact cardinals.) Let $\lambda=\lambda^{<\lambda}>\kappa_{n}, \theta_{l}<\lambda$. Then we blow up $2^{\kappa_{n}}$ to $\lambda$, change $\operatorname{cf}\left(\kappa_{n}\right)$ to $\theta_{n}$; blow up $2^{\kappa_{n-1}}$ to $\lambda$, change $\operatorname{cf}\left(\kappa_{n-1}\right)$ to $\sigma_{n-1}$, etc. The point is that when we arrive at $\kappa_{i}$ the forcing so far is fairly " $\kappa_{i}$-complete for pure extensions", etc., so does not destroy " $\kappa_{i}$ is $\lambda$-hypermeasurable". So for Conjecture 1.12 we (fix the desired cofinality $\theta$ and) need to do it not $n$ times but $\theta^{*}$ times 
$\left(\theta^{*}=\right.$ inverse order of $\left.\theta\right)$ so we need "anti-well-founded iteration". In other words, we have $\left\langle\kappa_{i}: i\langle\theta\rangle\right.$ increasing; $\kappa_{i}$ is $\lambda$-hypermeasurable indestructible (necessarily in a strong way), and $\lambda>\sum_{j<\theta} \kappa_{j}$.

First try. We may try to define, by induction on $i<\theta$, <-decreasing sequences $\overline{\mathbb{P}}^{i}=\left\langle\mathbb{P}_{j}^{i}: j \leq i\right\rangle$ of forcing notions such that $\left|\mathbb{P}_{0}^{i}\right|=\lambda_{i}$,

$$
\Vdash_{\mathbb{P}_{0}^{i}} \text { "cf }\left(\kappa_{j}\right)=\aleph_{0} \text { for } j<i "
$$

(or whatever fixed value, but $\aleph_{0}$ is surely easier), $\mathbb{P}_{j}^{i}$ is $\kappa_{j}^{+}$-c.c., purely $\kappa_{j^{-}}$ complete, $\mathbb{P}_{0}^{i}$ makes $\operatorname{pp}\left(\kappa_{j}\right)=\lambda$ for every $j<i$.

At successor stage - no problem: $i=j+1$ and

$\mathbb{P}_{i+1}^{i+1}=\left(\right.$ blowing up $2^{\kappa}$ to $\lambda$ changing $\operatorname{cf}\left(\kappa_{j}\right)$ to $\left.\aleph_{0}\right), \quad \mathbb{P}_{j}^{i+1}=\mathbb{P}_{i+1}^{i+1} * \mathbb{P}_{j}^{i}$.

Not good enough: $\mathbb{P}_{i}^{i+1}$ changes the definition of: "blowing up $2^{\kappa_{j}}$ to $\lambda$ " as there are more $\omega$-sequences. So we should correct ourselves to $\left|\mathbb{P}_{j}^{i}\right|=$ $\sum_{\zeta<i} \kappa_{\zeta}$

$$
\mathbb{P}_{j}^{i} \text { blows up } 2^{\kappa_{j}} \text { to essentially } \sum_{\zeta<i} \kappa_{\zeta} .
$$

So we have to prove the forcing notions extend as they should. If $\overline{\mathbb{P}}^{i}$ is defined, there is no problem to choose an appropriate $\mathbb{P}_{i+1}^{i+1}$. Now for each $j \leq i$ separately we would like to choose $\mathbb{P}_{j}^{i+1}$ to be a $<$-extension of $\mathbb{P}_{j}^{i}$ and of $\mathbb{P}_{i+1}^{i+1}$, but we have to do it for all $j \leq i$ together. The limit case seems harder.

Why, in $1.12(\mathrm{~A})$, do we have $\theta \geq \aleph_{1}$ ? Moti Gitik shows consistency for $\theta=\aleph_{0}$ by known methods.

Audience Question: How dare you conjecture ZFC can show 1.10, 1.11?

For Conjecture 1.12 I have a scenario for an independence proof (outlined above). For 1.10 and 1.11 the statements imply there is quite a complicated pcf structure you necessarily drag with you. So it is reasonable to assume that if we know enough theorems on the pcf structure we shall get a contradiction. Of course, those arguments are not decisive.

Traditionally, remnants of GCH have strongly influenced the research on cardinal arithmetic, so e.g. people concentrate on the strong limit case (see [166, AG], [98]); probably also it was clear what to do and easier. On 
the other hand, [166] aims to get "exponentiation-free theorems", so we put forward:

Thesis 1.13. "Everything" is expressible by cases of $\mathrm{pp}_{J}$ (and $2^{\kappa}$ for $\kappa$ regular).

E.g. in $[100, \S 2]$ this is done to the tree power of $\lambda$, $\lambda^{\langle\kappa, \operatorname{tr}\rangle}=\sup \left\{\left|\lim _{\kappa}(T)\right|: T\right.$ a tree with $\leq \lambda$ nodes and $\kappa$ levels $\}$,

where $\lim _{\kappa}(T)$ is the set of $\kappa$-branches of $T$ (well, using $\kappa^{\langle\kappa, \text { tr }\rangle}$ for regular $\kappa$, which is malleable by forcing, a relative of $2^{\kappa}$ for $\kappa$ regular).

But maybe there are also forcing proofs by which we can get interesting situations say below the continuum, whose strong limit counterparts are false, or have bigger consistency strength, or at least are harder to prove. The known forcing proofs may be open to such variations, e.g., when we add many Prikry sequences to one $\kappa$ we may have the order between them such that every condition decides little about it. The following problem may be relevant to 1.13 , and anyhow is a central one.

Problem 1.14. For a singular cardinal $\mu>\theta=\operatorname{cf}(\mu)$, is

$$
\operatorname{cov}\left(\mu, \mu, \theta^{+}, \theta\right)=\operatorname{pp}_{\Gamma\left(\theta^{+}, \theta\right)}(\mu) ?
$$

Note that other cases of cov can be reduced to those above. Now, this is almost proved: it holds when $\theta=\operatorname{cf}(\mu)>\aleph_{0}$. Furthermore, if $\mu$ is strong limit, $\aleph_{0}=\operatorname{cf}(\mu)$ and the two expressions in 1.14 are not equal, then both are quite large above $\mu$; there are quite some fixed points $>\mu$ below them; moreover fixed points which are counterexamples to Conjecture 1.12. Also, e.g., for a club of $\delta<\omega_{1}$,

$$
\operatorname{cov}\left(\beth_{\delta}, \beth_{\delta}, \aleph_{1}, \aleph_{0}\right)=\operatorname{pp}\left(\beth_{\delta}\right)
$$

(see [98], the " $\beth_{\omega_{1}}$ " can be weakened to strong limit in cov sense). But

Question 1.15. Can we force that there is $\mu<2^{\aleph_{0}}$ such that $\operatorname{cf}(\mu)=\aleph_{0}$ and $\operatorname{cov}\left(\mu, \mu, \aleph_{1}, \aleph_{0}\right)>\operatorname{pp}(\mu) ?$

$\left[\right.$ Why $<2^{\aleph_{0}}$ ? As blowing up the continuum does not change the situation, proving the consistency for $\mu<2^{\aleph_{0}}$ can be only easier. But for $\mu<2^{\aleph_{0}}$ maybe it is even consistent that

$$
\operatorname{cov}\left(\mu, \mu, \aleph_{1}, \aleph_{0}\right)>\mu^{+}=\operatorname{pp}(\mu),
$$

that is, by our present ignorance, it is even possible that the behaviour below the continuum is different than above it.]

Note that all cases of $\lambda^{\kappa}$ can be reduced to cases of $2^{\theta}, \theta$ regular, and $\operatorname{cf}([\mu] \leq \theta, \subseteq)$ where $\mu>\operatorname{cf}(\theta)=\theta \geq \operatorname{cf}(\mu)$. Why? If $\kappa$ is regular, $\lambda \leq 2^{\kappa}$ then $\lambda^{\kappa}=2^{\kappa}$. If $\kappa$ is regular and $\lambda>2^{\kappa}$ then $\lambda^{\kappa}=\operatorname{cf}([\lambda] \leq \kappa, \subseteq)$. So assume $\kappa$ is singular and let $\sigma=\operatorname{cf}(\kappa)$ and $\kappa=\sum_{i<\sigma} \kappa_{i}$, where each $\kappa_{i}$ is regular 
and $\sigma<\kappa_{i}<\kappa$, so $\lambda^{\kappa}=\lambda^{\sum\left\{\kappa_{i}: i<\sigma\right\}}=\prod_{i<\sigma} \lambda^{\kappa_{i}}$. Thus, if $\lambda \leq 2^{\kappa}$ then

$$
\lambda^{\kappa}=2^{\kappa}=\prod_{i<\sigma} 2^{\kappa_{i}}=\left(\sum_{i<\sigma} 2^{\kappa_{i}}\right)^{\sigma}=\operatorname{cf}\left(\left[\sum_{i<\sigma} 2^{\kappa_{i}}\right]^{\sigma}, \subseteq\right) .
$$

Lastly, if $\lambda>2^{\kappa}$, then

$$
\lambda^{\kappa}=\prod_{i<\sigma} \lambda^{\kappa_{i}}=\left(\sum_{i<\sigma} \lambda^{\kappa_{i}}\right)^{\sigma}=\left(\max _{i<\sigma} \lambda^{\kappa_{i}}\right)^{\sigma}=\max _{i<\sigma} \lambda^{\kappa_{i}}=\max _{i<\sigma} \operatorname{cf}\left([\lambda]^{\kappa_{i}}, \subseteq\right)
$$

(on the third equality see Hajnal and Hamburger [54] or Shelah [151, 2.11(4), p. 164]).

If the answer to 1.14 is yes, then we can reduce all cases of $\lambda^{\kappa}$ and of cov to statements on cases of pp.

Problem 1.16. If $\operatorname{cf}(\mu)=\aleph_{0}$, is $\operatorname{pp}(\mu)$ equal to $\operatorname{pp}_{J_{\omega}^{\mathrm{bd}}}^{\mathrm{cr}}(\mu)$, where $\operatorname{pp}_{J_{\omega}^{\mathrm{bd}}}^{\mathrm{cr}}(\mu)=\sup \left\{\lambda\right.$ : for some increasing sequence $\left\langle\lambda_{n}: n<\omega\right\rangle$ of regular cardinals converging to $\mu$ we have $\left.\lambda=\operatorname{tcf}\left(\prod_{n<\omega} \lambda_{n} / J_{\omega}^{\mathrm{bd}}\right)\right\}$ ? A variant is: except when $\operatorname{pp}_{J_{\omega}^{\mathrm{bd}}}^{\mathrm{cr}}(\mu)$ has cofinality $\aleph_{0}$ and $\operatorname{pp}(\mu)$ is its successor.

By pcf calculus, if $\operatorname{pp}(\mu)<\mu^{+\omega_{1}}$ then this is true. Similarly, if $\theta<\mu_{0}<$ $\mu$ and

$$
\left(\forall \mu^{\prime}\right)\left(\left[\operatorname{cf}\left(\mu^{\prime}\right) \leq \theta \& \mu^{\prime} \in\left(\mu_{0}, \mu\right) \Rightarrow \operatorname{pp}\left(\mu^{\prime}\right)<\mu^{+\theta^{+}}\right]\right.
$$

then $\operatorname{pp}(\mu)=\operatorname{pp}_{\theta}(\mu)$ and see [170,6.5]. Also, by [98, Part C], e.g., for a club of $\delta<\omega_{1}, \mu=\beth_{\delta}$ satisfies the conclusion.

On pcf for set theories with weak versions of Choice (say $\mathrm{DC}_{\kappa}$, the dependent choice of length $\kappa$ ) see [175].

Problem 1.17. Develop combinatorial set theory generally and, in particular, pcf theory using only little choice (say $\mathrm{DC}_{\kappa}$ ).

Inner model theory and descriptive set theory are not hampered by lack of choice, and much was done on variants of the axiom of choice. [175] may be a beginning of combinatorial set theory, and pcf in particular; i.e., it is enough to show that there are interesting theorems. In particular:

Question 1.18. (a) Does $\mathrm{DC}_{\kappa}$ for $\kappa$ large enough imply the existence of a proper class of regular cardinals?

(b) Does $\mathrm{DC}_{\kappa}$ for $\kappa$ large enough imply that for a class of $\lambda, \mathcal{P}(\lambda)$ is not the union of $<\lambda$ sets, each of cardinality $\leq \lambda$ ? 
See more in [175]. Gitik [41] proved

$$
\operatorname{CON}\left((\forall \delta)\left(\operatorname{cf}(\delta) \leq \aleph_{0}\right)\right)
$$

relative to suitable large cardinals. Woodin asked if

$$
\operatorname{CON}\left(\mathrm{DC}_{\aleph_{0}}+(\forall \delta)\left(\mathrm{cf}(\delta) \leq \aleph_{1}\right)\right)
$$

Specker asked if, consistently, for every $\lambda$, for some $\left\langle A_{n}: n<\omega\right\rangle$ we have $\mathcal{P}(\lambda)=\bigcup_{n} A_{n},\left|A_{n}\right| \leq \lambda$.

On how the problem of the existence of universal objects is connected to pcf see Kojman and Shelah [70], and [173], [117]. The following conjecture will simplify the answers:

Conjecture 1.19. For every limit of limit cardinals $\mu$, for arbitrarily large regular $\lambda<\mu$, we have

$$
\left(\forall^{*} \mu_{1}<\mu\right)\left[\operatorname{cf}\left(\mu_{1}\right)=\lambda \Rightarrow \operatorname{pp}_{\Gamma(\lambda)}\left(\mu_{1}\right)<\mu\right],
$$

where $\forall^{*}$ means "for every large enough".

After we learned that, on the one hand, $2^{\aleph_{\omega}}$ ( $\aleph_{\omega}$ strong limit) has a bound (in fact, every $2^{\aleph_{\delta}}$, if $\aleph_{\delta}$ is strong limit $>|\delta|$, in [142, Ch.XIII]), and on the other hand there are bounds for $2^{\mu}, \mu$ the $\omega_{1}$ th fix point (when $\mu$ is strong limit or less), it becomes natural to ask:

Conjecture 1.20. If $\aleph_{\delta}$ is the first fix point (i.e., the first such that $\aleph_{\delta}=\delta$, so it has cofinality $\left.\aleph_{0}\right)$, then $\operatorname{pp}\left(\aleph_{\delta}\right)<\left(2^{\aleph_{0}}\right)^{+}$th fix point.

(Even assuming GCH below $\aleph_{\delta}$ and proving just $\operatorname{pp}\left(\aleph_{\delta}\right)<$ "the first inaccessible" is good, but " $<\omega_{4}$ th fix point" is better, and " $<\omega_{1}$ th fix point" is best, but seems pointless to ask as long as 1.1 is open.)

Note that we almost know: if $\aleph_{\delta}$ is the $\omega_{1}$ th fix point (strong limit), then $\operatorname{pp}\left(\aleph_{\delta}\right)<\omega_{4}$ th fix point; we know it if the answer to 1.10 is yes: see [163] and $[166$, Ch.V].

Traditionally we have asked: "can we find all the laws of cardinal arithmetic?" This had been accomplished for regular cardinals, and we prefer

Problem 1.21. Find all the rules of the pcf calculus or at least find more (or show that the set of rules is inherently too complicated).

Note: if for simplicity $|\operatorname{pcf}(\mathfrak{a})|<\min (\mathfrak{a})$, then on $\operatorname{pcf}(\mathfrak{a})$ the pcf structure is naturally a compact topology: $\mathfrak{b}$ is closed iff $\mathfrak{b}=\operatorname{pcf}(\mathfrak{b})$, and the theorem on existence of generators $\left\langle\mathfrak{b}_{\lambda}: \lambda \in \operatorname{pcf}(\mathfrak{a})\right\rangle$ says that the topology is a 
particularly nice one. If 1.10 holds this is true whenever $|\mathfrak{a}|<\min (\mathfrak{a})$ (see $[97])$.

There may well be some "global phenomena". Also there may be special behaviour near

$\min \{\lambda$ : for some $A \subseteq \lambda$, there is no indiscernible class for $K[A]\}$,

as above it the covering theorem (Dodd and Jensen [27]) shows that cardinal arithmetic is trivial. On the other hand, on the behaviour below it, see [166, Ch. V]. is:

An extreme case of our non-understanding concerning global behavior

QUESTION 1.22. Is it possible that if $\mathfrak{a}$ is a set of odd [even] regular cardinals $>|\mathfrak{a}|$, then every $\theta \in \operatorname{pcf}(\mathfrak{a})$ is odd [even]? (Here $\aleph_{2 \alpha}$ is even and $\aleph_{2 \alpha+1}$ is odd.)

Instead of looking more on $\operatorname{pp}\left(\aleph_{\omega}\right)$ we may ask if the best result was derived from the known laws of cardinal arithmetic.

Question 1.23. Let $l<4$. Can there be $\delta \in\left[\omega_{l}, \omega_{l+1}\right)$ and a closure operation $c l$ on $\mathcal{P}(\delta+1)$ such that all the rules used in the proof of $\operatorname{pp}\left(\aleph_{\omega}\right)<$ $\aleph_{\omega_{4}}$ hold? (See Jech and Shelah [60].)

Question 1.24. (1) Characterize the possible sequences

$$
\left\langle J_{<\theta}\left[\left\{\aleph_{n}: n \in[1, \omega)\right\}\right]: \theta \in \operatorname{pcf}\left\{\aleph_{n}: n \in[1, \omega)\right\}\right\rangle .
$$

(2) For every ordinal $\gamma$ characterize the possible $\left\langle J_{<\theta}[\mathfrak{a}]: \theta \in \operatorname{pcf}(\mathfrak{a})\right\rangle$ up to isomorphism when $\operatorname{otp}(\mathfrak{a})=\gamma$.

For $\mathfrak{a}^{\prime}, \mathfrak{a}^{\prime \prime}$ we have an isomorphism if there is a one-to-one order preserving $f: \mathfrak{a}^{\prime} \rightarrow \mathfrak{a}^{\prime \prime}$ such that $\left\{J_{<\theta}\left[\mathfrak{a}^{\prime \prime}\right]: \theta \in \operatorname{pcf}\left(\mathfrak{a}^{\prime \prime}\right)\right\}=\left\{\left\{f[\mathfrak{b}]: \mathfrak{b} \in J_{<\theta}\left[\mathfrak{a}^{\prime}\right]\right\}\right.$ : $\left.\theta \in \operatorname{pcf}\left(\mathfrak{a}^{\prime}\right)\right\}$.] We may strengthen this demanding $\theta=\left(\sum_{i<\delta} \theta_{i}\right)^{+} \Rightarrow f(\theta)=$ $\left(\sum_{i<\delta} f\left(\theta_{i}\right)\right)^{+}$and $\theta=\sigma^{+} \Rightarrow f(\theta)=(f(\theta))^{+}$(i.e. essential continuity).

\section{I feel that}

Thesis 1.25. Proving a theorem from ZFC + "cardinal arithmetic assumptions" is a "semi-ZFC result".

This view makes proofs from cases of the failure of the $\mathrm{SCH}$ related to the thesis below more interesting.

Thesis 1.26. Assumptions on the failure of GCH (and even more so, of $\mathrm{SCH}$ ) are good assumptions, practical ones, in the sense that from them you can deduce theorems. 
Traditionally this is how instances of GCH were treated (with large supporting evidence). Clearly 1.26 may be supported by positive evidence (though hard to refute), whereas 1.25 remains a matter of taste. So Magidor would stress looking at "existence of a large cardinal" as semi-ZFC axioms (unlike some randomly chosen consistent theorems), which seems to mean in our terminology that we will look at consequences of it as semi-ZFC theorems. Jensen stresses that showing $\psi$ holds in a universe with (fine) structure is much better than mere consistency (so the fine structure in $\mathbf{L}$ was the only one we knew of at one time, but e.g. $K$ is no less good than $\mathbf{L}$; the statement in [108] was inaccurate).

I agree with both, just to a lesser degree. Kojman criticized 1.25 saying cases of failure of $\mathrm{SCH}$ are large cardinal assumptions in disguise; and I agree that $2^{\lambda}>\aleph_{\lambda+4}$ is a weaker assumption than $2^{\beth_{\omega}}>\beth_{\omega}^{+}$, but I still stick to 1.25 . We may hope to really resolve problems by partitioning to cases according to what the cardinal arithmetic is.

Discussion. The following should be obvious, but I have found that mentioning them explicitly is helpful. Assume, e.g., that $\operatorname{cf}(\mu)=\aleph_{0}$, $\operatorname{pp}(\mu)>\mu^{+\omega_{n}}, n>0\left(\omega_{n}\right.$ is chosen just for simplicity) and let

$(*)_{\mu, n} \quad$ for stationarily many $\delta<\omega_{n}$ of cofinality $\aleph_{0}, \operatorname{pp}_{\aleph_{n}}\left(\mu^{+\delta}\right)<\mu^{+\omega_{n}}$ (a "soft" assumption, see [166, IX, §4]).

Then we can find pairwise disjoint countable $\mathfrak{a}_{i} \subseteq \operatorname{Reg} \cap \mu$ unbounded in $\mu$ and $\alpha_{i}<\omega_{n}$ successor, strictly increasing and such that

$$
\mu^{+\alpha_{i}}=\max \operatorname{pcf}\left(\mathfrak{a}_{i}\right), \quad \mu^{+\alpha_{i}} \notin \operatorname{pcf}\left(\bigcup_{j \neq i} \mathfrak{a}_{j}\right),
$$

moreover $\mu^{\prime}<\mu \Rightarrow \mu^{\alpha_{i}}=\max \operatorname{pcf}\left(\mathfrak{a}_{i} \backslash \mu^{\prime}\right)$.

[Why? By the assumption and Fodor Lemma, we can find $\alpha^{*}<\omega_{n}$ such that (recalling that in $(*)_{\mu, n}$ we write $\mathrm{pp}_{\aleph_{n}}$ and not $\mathrm{pp}_{\aleph_{0}}$ )

$$
\alpha \in\left[\alpha^{*}, \omega_{n}\right) \Rightarrow \max \operatorname{pcf}\left\{\mu^{+\beta+1}: \beta \in\left(\alpha_{0}, \alpha\right)\right\}<\mu^{+\omega_{n}} .
$$

By the assumption $\operatorname{pp}(\mu) \geq \mu^{+\omega_{n}}$, there is $\mathfrak{a} \subseteq \mu \backslash \omega_{n}$ with $|\mathfrak{a}|=\aleph_{n}$ such that $\alpha<\omega_{n} \Rightarrow \mu^{+\alpha+1} \in \operatorname{pcf}(\mathfrak{a})$. First assume $2^{\aleph_{n}}<\mu$, so without loss of generality $\min (\mathfrak{a})>2^{\aleph_{n}}$, and we have a smooth closed generating sequence $\left\langle\mathfrak{b}_{\lambda}: \lambda \in \operatorname{pcf}(\mathfrak{a})\right\rangle$ for $\operatorname{pcf}(\mathfrak{a})\left(\operatorname{so} \mathfrak{b}_{\lambda} \subseteq \operatorname{pcf}(\mathfrak{a})\right.$, etc.). Now choose by induction on $i<\omega_{n}$ pairs $\left(\alpha_{i}, \mathfrak{b}_{i}^{\prime}\right), \alpha_{i}<\omega_{4}$, as follows. If $\left\langle\alpha_{j}: j<i\right\rangle$ has been defined, we know that

$$
\max \operatorname{pcf}\left\{\mu^{+\beta}: \beta \text { successor, } \alpha^{*} \leq \beta \leq\left(\alpha^{*}+2\right) \cup \bigcup_{j<i} \alpha_{j}\right\}<\mu^{+\omega_{n}},
$$


and hence we can find $m_{i}<\omega$ and successor ordinals

$$
\gamma_{l}^{i} \in\left[\alpha^{*}, \omega_{4}\right) \quad\left(\text { for } l<m_{i}\right)
$$

such that

$$
\left\{\mu^{+\beta}: \beta \text { a successor, } \alpha^{*} \leq \beta \leq\left(\alpha^{*}+1\right) \cup \bigcup_{j<i} \alpha_{j}\right\} \subseteq \bigcup_{l<m_{i}} \mathfrak{b}_{\gamma_{l}^{i}} .
$$

Let $\alpha_{i}<\omega_{n}$ be the minimal successor such that

$\mu^{+\alpha_{i}}>\max \operatorname{pcf}\left\{\mu^{+\beta}: \beta\right.$ a successor, $\left.\alpha^{*} \leq \beta \leq\left(\alpha^{*}+1\right) \cup \bigcup_{j<i} \alpha_{j} \cup \bigcup_{l<m_{i}} \gamma_{l}^{i}\right\}$,

and let $\mathfrak{a}_{i}=\mathfrak{b}_{\alpha_{i}} \backslash \bigcup_{l<m_{i}} \mathfrak{b}_{\gamma_{l}}$. If $\neg\left(2^{\aleph_{n}}<\mu\right)$ use the end of $[170, \S 6]$.]

If, changing $(*)_{\mu, n}$, we assume that for some $\alpha^{*}<\omega_{n}$ we have

$$
\delta>\alpha^{*} \& \delta<\omega_{n} \text { is limit } \Rightarrow \operatorname{pp}\left(\mu^{+\delta}\right)<\mu^{+\omega_{n}},
$$

then we can get the same conclusion. Of course, omitting $(*)_{\mu, n}$ if $2^{\aleph_{0}}<$ $\omega_{n}$, by the $\Delta$-system lemma, we can get $\left\langle\left(\mathfrak{a}_{i}, \alpha_{i}\right): i<\omega_{n}\right\rangle$ as above but demanding only $i \neq j \Rightarrow \mu^{+\alpha_{i}} \notin \operatorname{pcf}\left(\mathfrak{a}_{j}\right)$. Of course, we cannot let $\alpha_{i}=i+1$, as e.g. for some infinite $A \subseteq \omega, \mu^{+\omega+1}=\operatorname{tcf}\left(\prod_{n \in A} \mu^{+n} / J_{A}^{\mathrm{bd}}\right)$, and hence $\mu^{+\omega+1} \in \operatorname{pcf}\left(\bigcup_{n \in A} \mathfrak{a}_{n}\right)$

Another remark. Even if $\operatorname{pcf}(\mathfrak{a})$ is large and $\mathfrak{a}$ is countable, we can find a c.c.c. forcing notion $\mathbb{Q}$ such that in $\mathbf{V}^{\mathbb{Q}}$ we can find $\left\langle\mathfrak{b}_{\lambda}: \lambda \in \operatorname{pcf}(\mathfrak{a}) \backslash \mathfrak{a}\right\rangle$ satisfying: $\quad \mathfrak{b}_{\lambda} \subseteq \mathfrak{a}$ has order type $\omega$ and $\prod \mathfrak{b}_{\lambda} / J_{\mathfrak{b}_{\lambda}}^{\text {bd }}$ has true cofinality $\lambda$. [Why? If $\left\langle\mathfrak{b}_{\lambda}: \lambda \in \operatorname{pcf}(\mathfrak{a})\right\rangle$ is a generating sequence, let $\mathbb{Q}$ force for each $\lambda$ an $\omega$-sequence $\subseteq \mathfrak{b}_{\lambda}$, almost disjoint from $\mathfrak{b}_{\lambda_{1}}$ for $\lambda_{1}<\lambda$.] Such forcing does not change the pcf structure (in fact, if $\left\langle\mathfrak{b}_{\lambda}: \lambda \in \operatorname{pcf}(\mathfrak{a})\right\rangle$ is a generating sequence for $\mathfrak{a}$ and $\mathbb{Q}$ is a $\min (\mathfrak{a})$-c.c. forcing notion, then $\left\langle\mathfrak{b}_{\lambda}: \lambda \in \operatorname{pcf}(\mathfrak{a})\right\rangle$ is still a generating sequence for $\mathfrak{a}$, witnessed by the same $\left.\left\langle f_{\alpha}^{*}: \alpha<\lambda\right\rangle\right)$.

QUESTION 1.27. For a regular cardinal $\theta$, can we find an increasing sequence $\left\langle\lambda_{i}: i<\theta\right\rangle$ of regular cardinals such that for some successor $\lambda$ and $f_{\alpha} \in \prod_{i<\theta} \lambda_{i}$ for $\alpha<\lambda$ we have:

(*) if $C_{i}$ is a club of $\lambda_{i}$ for $i<\theta$, then for every large enough $\alpha<\lambda$ for every large enough $i<\theta$ we have $f_{\alpha}(i) \in C_{i}$ ?

By $[127, \S 6]$ an approximation to this holds: if $\mu$ is a strong limit singular cardinal, $\operatorname{pp}(\mu)={ }^{+} 2^{\mu}$ and $\lambda=2^{\mu}=\operatorname{cf}\left(2^{\mu}\right)$ then the answer is yes, i.e. (*) holds true, but $2^{\mu}$ may be a limit cardinal (if $2^{\mu}$ is singular, a related statement holds).

QUESTION 1.28. Assume $\kappa=\operatorname{cf}(\kappa),\left\langle\mu_{i}: i \leq \kappa\right\rangle$ is an increasing continuous sequence of strong limit cardinals $>\kappa$, for non-limit $i, \operatorname{cf}\left(\mu_{i}\right)=\aleph_{0}$ and $\prod_{i<\kappa} \mu_{i}^{+n} / J_{\kappa}^{\mathrm{bd}}$ has true cofinality $\mu^{+n}$. Can we find an interesting colouring theorem on $\mu^{+n}$ ? (The point is that for $n \geq 2$, we can have both a colouring 
as $\mu^{+n}$ is a successor of regulars (as in [158], [172]) and using a witness to $\operatorname{tcf}\left(\mu_{i}^{+n} / J_{\kappa}^{\mathrm{bd}}\right)=\mu^{+n}$ as in [103], [127].) The question is whether combining we shall get something startling.

Question 1.29. (1) Are there non-metrizable first countable Hausdorff topological spaces which are $\aleph_{2}$-metrizable (i.e., the induced topology on any $\leq \aleph_{1}$ points is metrizable)?

(2) Are there non-collectionwise Hausdorff, first countable Hausdorff topological spaces which are $\aleph_{1}$-collectionwise Hausdorff?

See [126]. Concerning hopes to answer yes note that if SCH fails (or just $\operatorname{cf}(\mu)=\aleph_{0}, \operatorname{pp}(\mu)>\mu^{+}$) then there are examples (see [126, $\left.\S 1\right]$ ), so we are allowed to assume $2^{\beth_{\omega}}=\beth_{\omega}^{+}$, etc.

Question 1.30. Let $D$ be an ultrafilter on $\kappa$ and $\operatorname{Spc}(D)=\left\{\prod_{i<\kappa} \lambda_{i} / D\right.$ : $\lambda_{i} \geq 2^{\kappa}$ for $\left.i<\kappa\right\}$. Is $\operatorname{Spc}(D)$ equal to $\left\{\mu: 2^{\kappa} \leq \mu=\mu^{<\operatorname{reg}(D)}\right\}$ ? (Here $\operatorname{reg}(D)=\sup \left\{\theta\right.$ : for some $A_{i} \in D, i<\theta$, for every $\alpha<\kappa$, the number of $i<\theta$ such that $\alpha \in A_{i}$ is finite $\}$.)

See on this [100] where some information is gained.

Question 1.31. For which $\lambda \geq \mu$ can we find an almost disjoint family $\mathcal{A} \subseteq[\lambda]^{\aleph_{0}}$ such that

$$
\left(\forall X \in[\lambda]^{\mu}\right)(\exists A \in \mathcal{A})\left(A \subseteq^{*} X\right) ?
$$

At least when $\lambda \geq \mu=\beth_{\omega}$ ? (See [178], [99].)

QUESTION 1.32. Is it consistent that for some strong limit singular cardinal $\mu$, for no regular $\lambda \in\left[\mu, 2^{\mu}\right]$ do we have a c.c.c. Boolean algebra which is not $\lambda$-Knaster?

On related ZFC constructions see [103], [127]; see also $\S 6$ here.

QUESTION 1.33. Are all the assumptions in the result of [128] (see below) necessary? In particular, are assumptions (a), (b), (c) below sufficient?

Theorem 1.34 (see [128]). Assume that:

(a) $\mathbf{V}$ is our universe of sets, $\mathbf{W}$ is another model of $Z F C$ (i.e., a transitive class of $\mathbf{V}$ containing all the ordinals),

(b) $\kappa$ is a regular cardinal in $\mathbf{V}$,

(c) $(\mathbf{W}, \mathbf{V})$ has $\kappa$-covering (that is, every set of $<\kappa$ ordinals from $\mathbf{V}$ is included in a set of $<\kappa$ ordinals from $\mathbf{W}$ ),

(d) the successor of $\kappa$ in $\mathbf{V}$ is the same as its successor in $\mathbf{W}$, call it $\kappa^{+}$,

(e) $(\mathbf{W}, \mathbf{V})$ has $\kappa^{+}$-covering.

Then $(\mathbf{W}, \mathbf{V})$ has the strong $\kappa$-covering (that is, for every structure $M$ with universe an ordinal $\alpha$ and a countable vocabulary, and a set $X$ from $\mathbf{V}$ of 
cardinality $<\kappa$, there is a set $Y$ from $\mathbf{W}$ of cardinality $<\kappa$ including $X$ which is the universe of an elementary submodel of $M)$.

2. The quest for the test: on the theory of Iterated Forcing for the continuum. On the subject see [176], and recent papers, too, but this section is hampered by some work in progress.

The issue is:

Problem 2.1. (a) Assuming we know something about each iterand $\mathbb{Q}_{i}$, what can we say about $\mathbb{P}_{\alpha}$, where $\left\langle\mathbb{P}_{i}, \mathbb{Q}_{j}: i \leq \alpha, j<\alpha\right\rangle$ is an iteration (which may be FS (finite support), or CS (countable support) or RSC (revised countable support) and more)?

(b) Find more useful ways to iterate (say, new "supports").

So "c.c.c. is preserved by FS iteration", "properness is preserved by CS iteration" can be seen as prototypes. But also many times: "adding no Cohen real over V", "adding no dominating real over V", etc., and, very natural, "adding no new real".

Note that this is not the same as having forcing axioms, e.g., having (the very important) MA does not discard the interest in FS iterations of c.c.c. forcing. The point is that in many questions you want to add reals for some purpose (which appear as generic sets for some forcing notions), but not another (e.g., a well-ordering of $\omega$ of order type $\omega_{1}$ ). Also considering an axiom speaking on forcing notions with some property, when considering a candidate, a forcing notion $\mathbb{Q}$, during an iteration we may force that it will not satisfy the property, discard it instead "honestly" forcing with it.

What we get by iterations as above can be phrased as having some axioms, but we have many combinations of adding reals of kinds $\mathrm{A}, \mathrm{B}$, and $\mathrm{C}$ while preserving properties $\mathrm{Pr}_{1}, \mathrm{Pr}_{2}$, in other words practically one preservation theorem may be used in many such contexts.

In fact, some of the most intriguing problems are fine distinctions: adding solution to one kind, but not to a close variant, e.g., the old problem:

Question 2.2. $\operatorname{CON}(\mathfrak{p}<\mathfrak{t})$ ? (Note that if $\mathfrak{p}<\mathfrak{t}$ then $2^{\aleph_{0}} \geq \aleph_{3}$. See 3.7.)

With FS iteration, all values of the continuum were similar, except $\aleph_{1}$ (well, also there is a distinction between regular and singular).

In fact, the advances in proper forcing make us "rich in forcing" for $2^{\aleph_{1}}=$ $\aleph_{2}$, making the higher values more mysterious. (So in [176, Chs. VII, VIII] we separate according to the size of the $\mathbb{Q}_{i}$ 's and whether we add reals, but we concentrate on the length $\omega_{2}$.) So, because we know much more how to force to get $2^{\aleph_{0}}=\aleph_{2}$, the independence results on the problems of the interrelation of cardinal invariants of the continuum have mostly dealt with relationships 
of two cardinals, as their values are $\in\left\{\aleph_{1}, 2^{\aleph_{0}}\right\}$. Thus, having only two possible values $\left\{\aleph_{1}, \aleph_{2}\right\}$, among any three two are equal; the Pigeonhole Principle acts against us. As we are rich in our knowledge to force for $2^{\aleph_{0}}=\aleph_{2}$, naturally we are quite poor concerning ZFC results. If we try for cardinal invariants $\mathbf{c}_{1}, \mathbf{c}_{2}$ to prove they consistently are $\aleph_{1}, \aleph_{2}$, respectively, much of our way exists (quoting existing preservation theorems) and we can look at the peculiarities of those invariants which may be still intractable. We are not poor concerning forcing for $2^{\aleph_{0}}=\aleph_{1}$ (and are rich in ZFC). But for $2^{\aleph_{0}} \geq \aleph_{3}$ we are totally lost: very poor in both directions. We would like to have iteration theory for length $\geq \omega_{3}$. I tend to think good test problems will be important in developing such iterations.

In some senses, most suitable is

Problem 2.3. Investigate cardinal invariants of the continuum showing $\geq 3$ may have prescribed order.

Of course, the lack of forcing ability does not stop you from proving hopeful ZFC theorems about them, if true. Now I think there are some, but:

Thesis 2.4. They are camouflaged by the independent statements.

[Yes, I really believe there are interesting restrictions.] However, once we prove 90 percent of the problems are independent we will know where to look (as, in hindsight, occurs in cardinal arithmetic). So cardinal invariants from this perspective are excellent excuses to find iteration theorems. Mainly for $2^{\aleph_{0}} \geq \aleph_{3}$, but, of course, there is more to be said on $2^{\aleph_{0}}=\aleph_{1}$ (though not for 2.3), and even $2^{\aleph_{0}}=\aleph_{2}$.

Without good test problems you are in danger of imitating the king who painted the target after shooting the arrow. Let us consider some additional well known problems:

Question 2.5 (see Just, Mathias, Prikry and Simon [66]). Is there a filter $D$ on $\omega$ such that:

(a) every co-finite subset of $\omega$ belongs to $D$,

(b) $D$ is a $P$-filter (i.e., if $A_{n} \in D$ for $n<\omega$, then for some $A \in D$, $\left.n<\omega \Rightarrow A \subseteq^{*} A_{n}\right)$,

(c) $D$ is not feeble, i.e., if $0=n_{0}<n_{1}<\ldots$, then for some $A \in D$ for infinitely many $i<\omega$ we have $\left[n_{i}, n_{i+1}\right) \cap A=\emptyset$ ?

Question 2.6 (see Garcia-Ferreira and Just [40]). Is there an almost disjoint family $\mathcal{A} \subseteq[\omega]^{\aleph_{0}}$ (i.e., $(\forall A \neq B \in \mathcal{A})\left[|A \cap B|<\aleph_{0}\right]$ ) of cardinality $\mathfrak{b}$ satisfying the following condition:

- if $A_{n} \in \mathcal{A}$ are pairwise distinct and $h: \omega \rightarrow \omega$ then for some $B \in \mathcal{A}$ we have $\left(\exists^{\infty} n\right)\left(A_{n} \cap B \nsubseteq h(n)\right)$ ?

If not, then $2^{\aleph_{0}}>\aleph_{\omega}$; on both questions see the discussion after 2.13 . 
Question 2.7 (see van Mill [195, Problem 4, p. 563], Miller [83, Problem 9.1]). CON(no $P$-point and no $Q$-point) ?

If so, $2^{\aleph_{0}} \geq \aleph_{3}$. [Why? Mathias [78] showed that if $\mathfrak{d}$ (the minimal size of a dominating family) is $\aleph_{1}$, then there is a $Q$-point. Ketonen [69] showed that $\mathfrak{d}=2^{\aleph_{0}}$ implies the existence of $P$-points.]

Question 2.8. $\mathrm{CON}(\omega(\omega+1)$ with box product topology is not paracompact) ?

If so, $2^{\aleph_{0}} \geq \aleph_{3}$. See on this Williams [197].

Question 2.9 (see Miller [83, Problem 16.3]). CON(Borel Conjecture and Dual Borel Conjecture) ? (See 3.3.)

Question 2.10. CON $(\operatorname{cf}(\operatorname{cov}$ (meagre) $)<\operatorname{additivity(meagre)})$ ? (See before 2.14.)

Problem 2.11. (1) CON(every function $f:{ }^{\omega} 2 \rightarrow{ }^{\omega} 2$ is continuous when restricted to some non-null set) ? [Here "null" means of Lebesgue measure zero.]

(2) Similarly for other natural ideals. This in particular means if $\mathbb{Q}$ is a nicely defined forcing notion (see $\S 5$ below, e.g., Suslin c.c.c.), $\eta$ a $\mathbb{Q}$-name of a real, $A \subseteq{ }^{\omega} 2$ is called $(\mathbb{Q}, \eta)$-positive if for every countable $\tilde{N} \prec(\mathcal{H}(\chi), \in$, $\left.<_{\chi}^{*}\right)$ to which $\mathbb{Q}, \underset{\sim}{\eta}$ belong, some $\eta \in A$ is $\eta[G]^{N}$ for some $G \subseteq \mathbb{Q}^{N}$ generic over $N$; so the question for such $\mathbb{Q}$ is: "CON(every $f:{ }^{\omega} 2 \rightarrow{ }^{\omega} 2$ has a continuous restriction to some $(\mathbb{Q}, \eta)$-positive set $A)$ ?

(3) Is the following consistent: if $A \subseteq{ }^{\omega_{2}}$ is non-null, $f: A \rightarrow{ }^{\omega_{2}}$ then for some positive $B \subseteq A, f\lceil B$ is continuous? Similarly for general ideals as in part (2).

(4) If $A \subseteq{ }^{\omega_{2}} \times{ }^{\omega_{2}} 2$ is not equivalent to a Borel set modulo one ideal $I_{1}$ (as described in part (2) above), then for some continuous $f:{ }_{2}{ }_{2} \rightarrow{ }^{\omega_{2}} 2$, the set $\left\{\eta \in{ }^{\omega} 2:(\eta, f(\eta)) \in A\right\}$ is not equivalent to a Borel set modulo another ideal $I_{0}$ for suitable pairs $\left(I_{0}, I_{1}\right)$.

See Fremlin [37], Ciesielski [20, Theorem 3.13, Problem 5]; [169] shows "yes" for (2) for non-meagre, Ciesielski and Shelah [21] prove "yes" for (4) for non-meagre, on work in progress see Rosłanowski and Shelah [88, §2]. With Juris Steprāns we have had some discussions on trying to use the oracle cc to the case of non-meagre ideal in (3). See 3.8 .

Note: Mathematicians who are not set theorists generally consider "null" as senior to "meagre", that is, as a more important case; set theorists inversely, as set-theoretically Cohen reals are much more manageable than random reals and have generalizations, relatives, etc. Particularly, in FS iterations, we get Cohen reals "for free" (in the limit), which kills our chances 
for many things and until now we have nothing parallel for random reals (but see [88]).

Judah suggests:

QUESTION $2.12(\mathbf{V}=\mathbf{L})$. Find a forcing making $\mathfrak{d}=\aleph_{3}$ but not adding Cohen reals.

I am skeptical whether this is a good test question, as you may make $\mathfrak{d}=\aleph_{3}=\mathfrak{b}$ by c.c.c. forcing, then add $\aleph_{1}$ random reals $\left\langle\nu_{i}: i<\omega_{1}\right\rangle$ by a measure algebra; so over $\mathbf{L}\left[\left\langle\nu_{i}: i<\omega_{1}\right\rangle\right]$ we have such a forcing. But certainly "not adding Cohen" is important, as many problems are resolved if $\operatorname{cov}($ meagre $)=2^{\aleph_{0}}$.

There is a basic question for us:

Problem 2.13. Is there an iteration theorem solving all the problems described above or at least for all cases involving large continuum not adding Cohen reals?

I suspect not, and the answers will be ramified.

Let us review some problems. Now, Problems 2.5, 2.6 are for $2^{\aleph_{0}}>\aleph_{\omega}$, as: in 2.5 , if $\operatorname{cf}\left([\mathfrak{d}]^{\aleph_{0}}, \subseteq\right)=\mathfrak{d}$ then there is such a filter (see [66]), and also in 2.6, if $\operatorname{cf}\left([\mathfrak{b}]^{\aleph_{0}}, \subseteq\right)=\mathfrak{b}$ there is a solution (see Just, Mathias, Prikry and Simon [66]).

It may well be that the solution will look like: let $\mu$ be a strong limit singular cardinal with $2^{\mu} \geq \mu^{++}>\mu^{+}$and we use FS iteration of length $<2^{\mu}$. This will be great, but probably does not increase our knowledge of iterations. If on the other hand along the way we will add new $\omega$-sequences say to $\mu\left(\operatorname{say} \operatorname{cf}(\mu)=\aleph_{0}\right)$ and necessarily we use more complicated iteration, then it will involve better understanding of iterations, probably new ones.

We can ([176, Ch. XIV]) iterate up to "large" $\kappa$, and for many $\alpha<\kappa, \alpha$ strongly inaccessible, we make $\mathbb{Q}_{\alpha}$ change its cofinality to $\aleph_{0}$. Sounds nice, but no target yet.

We may note that "FS iterations of c.c.c. forcing notions" is not dead. Concerning 2.3 and 2.10, there are recent indications that FS iteration of c.c.c. still can be exploited even in cases for which for a long time we thought new supports were needed. We can iterate with $\mathrm{FS},\left\langle\mathbb{P}_{i}, \mathbb{Q}_{i}: i<\alpha\right\rangle$, where $\mathbb{Q}_{i}$ is (partially random or is Cohen) adding a generic real $r_{i}, \mathbb{Q}_{i}$ is Cohen forcing or random forcing over $\mathbf{V}\left[\left\langle r_{j}: j \in A_{i}\right\rangle\right]$, where $A_{i} \subseteq i$ and each $\mathbb{Q}_{i}$

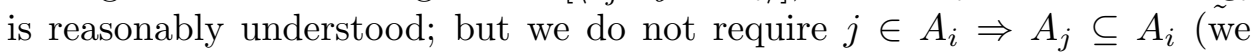
call it transitive memory). It is not so immediate to understand this sort of iterations, e.g., can the iteration add a dominating real? 
It appears that if the $A_{i}$ 's are sufficiently closed, it will not: see [105] used for $\operatorname{CON}(\operatorname{cov}($ null $))=\aleph_{\omega}$, more generally look at [130]. There we prove: $\operatorname{CON}\left(\exists\right.$ non-null $A$ such that the null ideal restricted to $A$ is $\aleph_{1}$-saturated).

Clearly we should use a measurable cardinal $\kappa$, a normal ultrafilter $\mathcal{D}$ on $\kappa$ in $\mathbf{V}$ and we add $\kappa$ random reals $\left\langle r_{\zeta}: \zeta<\kappa\right\rangle$, but how do we make

$$
A \in \mathcal{D}^{\mathbf{V}} \Rightarrow\left\{r_{\zeta}: \zeta \in \kappa \backslash A\right\} \text { is null ? }
$$

Cohen forcing does the job, but unfortunately too strongly. (In the case with non-meagre, earlier by Komjáth [72], there are no problems in this respect.) The solution is that we use FS iteration, but first we add $2^{\kappa}$ Cohens, that is, $\mathbb{Q}_{i}$ is Cohen forcing for $i<2^{\kappa}$; only then do we add the (somewhat) random reals:

$\mathbb{Q}_{\lambda+\zeta}$ for $\zeta<\kappa$ is random forcing over $\left\langle r_{\lambda+\xi}: \xi\langle\zeta\rangle^{\wedge}\left\langle r_{i}: i \in A_{\zeta} \subseteq \lambda\right\rangle\right.$.

We use $\left\{r_{i}: i<\lambda\right\}$ such that: $r_{i}$ makes $\left\{r_{\lambda+\zeta}: \zeta\right.$ satisfies $\left.i \in A_{\zeta}\right\}$ null for $i<2^{\aleph_{0}}$. So we need: for $A \in \mathcal{D}^{\mathbf{V}}$ for some $i<\lambda$ for every $\xi<\kappa$, $\xi \in \kappa \backslash A \Leftrightarrow i \notin A_{\xi}$.

This works for specially chosen $A_{\zeta}$ 's.

Problem 2.14. (1) Can you make this into a general method?

(2) Can you deal with $n$ or even $\kappa$ kinds of "reals" (getting interesting results)?

What does this mean? It means that we use FS iteration $\left\langle\mathbb{P}_{i}, \mathbb{Q}_{j}\right.$ : $i \leq \delta, j<\delta\rangle$ and $h: \delta \rightarrow \beta$, for $\zeta<\beta, \mathbb{R}_{\zeta}$ is a nep c.c.c. forcing notion in $\mathbf{V}$ (on nep see $\S 5$ and for more see [115]; e.g., $\mathbb{R}_{\zeta}$ is Cohen, random, or as in [90], [89] or whatever), and ${\underset{\sim}{\zeta}}_{\zeta} \in{ }^{\omega} 2$ is a generic real for $\mathbb{R}_{\zeta}$, and $\mathbb{Q}_{i}$ is $\mathbb{R}_{h(\zeta)}$ as interpreted in $\mathbf{V}\left[\left\langle{\underset{\sim}{j}}_{j}: j \in A_{i}\right\rangle\right]$ and $\underset{\sim}{r_{j}}$ is $\underset{\sim}{s_{h(j)}}$ there, and $A_{i} \subseteq i$. So the idea is that $0=\delta_{0}<\delta_{i}<\ldots<\delta_{n}=\delta$ and $j \in\left[\delta_{l}, \delta_{l+1}\right) \Rightarrow h(j)=l$.

In [109] we use $\aleph_{\varepsilon}$-historic support. This is less than $\left(<\aleph_{1}\right)$-support (i.e., countable support). This looks quite special, but

Problem 2.15. Can we make a general (interesting) theorem?

We can note that long FS iterations not only add Cohen reals, they also add, e.g., $\aleph_{2}$-Cohens, i.e. generics for $\left\{f: f\right.$ a finite function from $\omega_{2}$ to $\{0,1\}\}$. So we may like to iterate, allowing addition of Cohen reals but not $\aleph_{2}$-Cohens in the sense above. This is done in [109], but the family of allowable iterands can be probably widened. 
If we agree that preservation theorems are worthwhile, then after not collapsing $\aleph_{1}$, probably the most natural case is adding no reals. Now, whereas properness seems to me both naturally clear and covers considerable ground for not collapsing $\aleph_{1}$ and there are reasonable preservation theorems for "proper $+X$ " for many natural properties $X$ (e.g., adding no dominating reals, see $[176$, Ch. VI, $\S 1, \S 2, \S 3],[176$, Ch. XVIII, $\S 2])$, the situation with NNR (no new real) is inherently more complicated. In the early seventies when I heard on Jensen's $\mathrm{CON}(\mathrm{GCH}+\mathrm{SH})$, I thought it would be easy to derive an axiom; some years later this materialized as Abraham, Devlin and Shelah [6], but reality is not as nice as dreams. One obstacle is the weak diamond, see Devlin and Shelah [26], more in [142, Ch. XIV, §1], [176, AP, $\S 1],[113]$. For a time the iteration theorem in $[142, \mathrm{Ch} . \mathrm{V}, \S 5, \S 7$, Ch. VIII, §4] seemed satisfactory to me. [There we use two demands. The first was $\mathbb{D}$-completeness (this is a "medicine" against the weak diamond, and $\mathbb{D}$ is a completeness system, $\aleph_{1}$-complete in $[142, \mathrm{Ch} \mathrm{V}, \S 5,7]$, that is, any countably many demands are compatible, and just 2-complete in [142, Ch. VIII, §4], that is, any two demands are compatible). The second demand was $\alpha$-properness for each countable ordinal $\alpha$ (or relativized version, see $[142$, Ch. VIII, §4], [176, Ch. VIII, §4]).] But [148, §1] (better [176, Ch. XVIII, $\S 1])$ gives on the one hand very nice and easy forcing notions not adding reals (running away from club guessing sequences) which are not covered as they fail $\left(<\omega_{1}\right)$-properness and on the other hand, shows by a not so nice example that generally you cannot just omit the $\left(<\omega_{1}\right)$-properness demand and promise an iteration theorem covering them. The problem concerning that forcing was resolved (promised in [148], carried out in a different way in lectures in MSRI '89 = [176, Ch. XVIII, §2]), but resulted in a dichotomy: we can get by forcing $\mathrm{CON}(\mathrm{ZFC}+\mathrm{CH}+\mathrm{SH})$ and we can get by forcing $\mathrm{CON}(\mathrm{ZFC}+\mathrm{CH}+$ no club guessing $)$, but can we have both? More generally, can we have two other such contradictory statements? (More generally for such results see Shelah and Zapletal [187].)

Question 2.16. Can we have two statements of the form $\left({ }^{2}\right)$

$$
\left(\forall x \in \mathcal{H}\left(\aleph_{2}\right)\right)\left(\exists y \in \mathcal{H}\left(\aleph_{2}\right)\right) \varphi
$$

each consistent with

$$
\begin{aligned}
& \mathrm{CH}+\left[\operatorname { A x i o m } \left(\mathbb{Q} \text { is }\left(<\omega_{1}\right) \text {-proper and } \mathbb{D}\right.\right. \text {-complete } \\
& \text { for some simple } 2 \text {-completeness system } \mathbb{D})]
\end{aligned}
$$

but not simultaneously?

(We may change the axiom used, we may speak directly about the iteration; we may deal with CS and proper or with RCS and semi-proper, etc.)

\footnotetext{
$\left(^{2}\right) \mathcal{H}(\lambda)$ is the family of sets with transitive closure of cardinality $<\lambda$.
} 
Note: possible failure of iteration does not prove a ZFC consequence, we may have freedom in the iteration only in some stages (like c.c.c. productive under MA).

This leaves me in bad shape: the iteration theorems seem not good enough, but the test problem (of getting both) does not seem so good. Now, [120] deals with NNR solving the specific dichotomy (and really satisfies the [148] promise circumvented in [176, Ch. XVIII, §2]) but left 2.15 open.

Eisworth suggested to me (motived by Abraham and Todorčević [4])

QUESTION 2.17. Is the following consistent with $\mathrm{ZFC}+\mathrm{CH}$ :

(*) if $A_{\alpha} \in\left[\omega_{1}\right]^{\aleph_{0}}$ and $\alpha<\beta \Rightarrow A_{\alpha} \subseteq A_{\beta} \bmod$ finite, and for every stationary $S \subseteq \omega_{1}$ the set $\bigcup\left\{\left[A_{\alpha}\right]<\bar{\aleph}_{0}: \alpha \in S\right\}$ contains $[E]<\aleph_{0}$ for some club $E$ of $\omega_{1}$, then for some club $C$ of $\omega_{1}$ we have

$$
\left(\forall \alpha<\omega_{1}\right)\left(\exists \beta<\omega_{1}\right)\left(C \cap \alpha \subseteq A_{\beta}\right) ?
$$

For long (since [148]), an exciting problem for me has been

Problem 2.18. (1) Can we find a consequence of $\mathrm{ZFC}+\mathrm{CH}$ which "stands behind" the "club objection to NNR", e.g. it implies the failure of $\mathrm{CH}+\operatorname{Axiom}(\mathbb{Q}$ proper $\mathbb{D}$-complete for some single 2-completeness system $)$ ?

(2) Similarly for other limitations on iteration theorems.

Question 2.19. Is " $\mathrm{CH}+\mathcal{D}_{\omega_{1}}$ is $\aleph_{2}$-saturated" consistent, where $\mathcal{D}_{\omega_{1}}$ is the club filter on $\omega_{1}$ ?

Recall that a filter $D$ on a set $A$ is $\lambda$-saturated if there are no $A_{i} \in D^{+}$ for $i<\lambda$ such that $i<j \Rightarrow A_{i} \cap A_{j}=\emptyset \bmod D$.

See $[176, \mathrm{Ch} . \mathrm{XVI}]$. Woodin proved that if there is a measurable cardinal then no. So we may look at $L[A], A \subseteq \kappa$ codes $\mathcal{H}(\chi), \kappa$ large and try to collapse it to $\omega_{2}$.

Note that by [113], if $\mathrm{CH}+\mathcal{D}_{\omega_{1}}$ is $\aleph_{2}$-saturated, then essentially we have the weak diamond for three colours (or any finite number), but a strong negation of this is consistent with $\mathrm{CH}([176, \mathrm{Ch}$. VIII, §4]).

Baumgartner [11] asked

QUESTION 2.20. Is it consistent that $2^{\aleph_{0}}>\aleph_{2}$ and any two $\aleph_{2}$-dense subsets of $\mathbb{R}$ of cardinality $\aleph_{2}$ (that is, any interval has $\aleph_{2}$ points) are isomorphic (as linear orders)?

I think it is more reasonable to try

QUESTION 2.21. Is it consistent that $2^{\aleph_{0}}>\lambda \geq \aleph_{2}$ and there are no two far subsets $A \in[\mathbb{R}]^{\lambda}$ (see definition below)? 
Definition 2.22. The (linear orders) $I, J$ are $\theta$-far if there is no linear order of cardinality $\theta$ embedded into both. If $\theta$ is omitted, we mean $\min \{|I|,|J|\}$.

On $\mathrm{OCA}^{\prime}$ (i.e., $\mathrm{OCA}_{\aleph_{1}, \aleph_{0}}^{\prime}$, see the definition below) see Abraham, Rubin and Shelah [2], continued for $\mathrm{OCA}_{\aleph_{1}, \aleph_{0}}^{\prime \prime \prime}$ in Todorčević [194], Veličković [196]; on a parallel for subsets of the plane which follows from MA, see Steprāns and Watson [192].

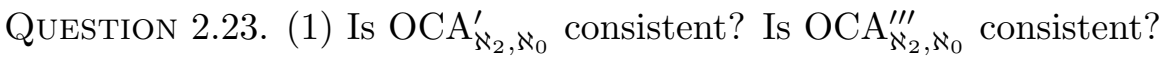

(2) The parallel problems for ${ }^{\kappa} 2$ and $\lambda$, even for $\lambda=\kappa^{+}, \kappa>\aleph_{0}$.

Definition 2.24. (1) $\mathrm{OCA}_{\lambda, \kappa}^{\prime}$ means $\lambda \leq 2^{\kappa}$ and: for any $A \in\left[{ }^{\kappa} 2\right]^{\lambda}$ and an open symmetric set $\mathcal{U} \subseteq{ }^{\kappa} 2 \times{ }^{\kappa} 2$ there is $B \subseteq A$ of cardinality $\lambda$ such that $\{(a, b): a \neq b$ are from $B\}$ is included in $\mathcal{U}$ or is disjoint from $\mathcal{U}$ (we use the space ${ }^{\kappa} 2$ for simplicity).

(2) $\mathrm{OCA}_{\lambda, \kappa}^{\prime \prime}$ is defined similarly but we have $B_{i} \subseteq A$ for $i<\kappa, A=$ $\bigcup_{i<\kappa} B_{i}$, each $B_{i}$ as in part (1).

(3) $\mathrm{OCA}_{\lambda, \kappa}^{\prime \prime \prime}$ means that $\lambda \leq 2^{\kappa}$ and for every open symmetric set $\mathcal{U} \subseteq$ ${ }^{\kappa} 2 \times{ }^{\kappa} 2$ there is $B \subseteq A$ of cardinality $\lambda$ such that $a \neq b \in B \Rightarrow(a, b) \in \mathcal{U}$ or there are $B_{i}$ for $i<\kappa$ such that $A=\bigcup_{i} B_{i}$ and $a \neq b \in B_{i} \Rightarrow(a, b) \notin \mathcal{U}$.

(4) If we omit $\lambda$ we mean $\lambda=\kappa^{+}$, if in addition we omit $\kappa$, we mean $\kappa=\aleph_{0}$.

3. Case studies for iterated forcing for the reals. The following was suggested during the lecture on $\S 2$ by Juhász who was in the audience:

Question 3.1. Does CH imply that there is an $S$-space of cardinality $\aleph_{2}$, where $S$-space is defined as being regular, hereditarily separable, not Lindelöf?

Eisworth prefers the variant: Does $\mathrm{CH}$ imply the existence of a locally compact $S$-space?

This problem looks important, but it is not clear to me if it is relevant to developing iteration theorems, though an existence proof may be related to the weak diamond, consistency to NNR iterations.

The same goes for the well known:

Question 3.2. $\operatorname{CON}(\mathfrak{d}<\mathfrak{a})$ ?

This definitely seems not to be connected to the iteration problem. It seems to me that a good test problem for our purpose in $\S 2$ should have one step clear but the iteration problematic, whereas for those two problems the situation is the inverse.

Note: by existing iteration theorems to get the consistency of $\mathfrak{d}<\mathfrak{a}$ $+2^{\aleph_{0}}=\aleph_{2}$ it is enough to show 
(*) for any MAD family $\left\{A_{i}: i<i^{*}\right\} \subseteq[\omega]^{\aleph_{0}}$, there is an ${ }^{\omega} \omega$-bounding proper forcing notion $\mathbb{Q}$ of cardinality $\aleph_{1}$ adding $\underset{\sim}{A} \in[\omega]^{\aleph_{0}}$ almost disjoint from each $A_{i}$.

You are allowed to assume $\mathrm{CH}$ (start with $\mathbf{V}=2^{\aleph_{0}}=\aleph_{1}+2^{\aleph_{1}}=\aleph_{2}$ and use CS iteration of such forcing notions); even $\nabla_{\aleph_{1}}$ (if $\mathbf{V} \models \diamond_{\aleph_{1}}$ ). We can weaken $|\mathbb{Q}|=\aleph_{1}$ to "Q্Q satisfies $\aleph_{2}$-pic" (this is a strong form of $\aleph_{2}$-c.c. good for iterating proper forcing, see [176, Ch. VIII, 2.1, p. 409]). If you agree to use large cardinals, it is okay to assume in $(*)$ that an appropriate forcing axiom holds and not restrict $|\mathbb{Q}|$, and as we can first collapse $2^{\aleph_{0}}$ to $\aleph_{1}$, we can get $\nabla_{\aleph_{1}}$ for "free". I idly thought to use free forcing for the problem ([176, Ch. IX] $]$, but no illumination resulted.

We can try in another way: start with a universe with a forcing axiom (say MA) and force by some $\mathbb{P}$ which makes $\mathfrak{d}=\aleph_{1}$, but $\mathbb{P}$ is understood well enough and we can show that $\mathfrak{a}$ is still large (just as adding a Cohen real to a model of MA preserves some consequences of MA (see Roitman [85], Judah and Shelah [61]). So clearly FS iteration will not do.

I think that a more interesting way is to consider, assuming $\mathrm{CH}$,

$$
\begin{aligned}
& K_{\omega_{1}}=\left\{(\overline{\mathbb{P}}, \bar{\sim}): \overline{\mathbb{P}}=\left\langle\mathbb{P}_{i}: i<\omega_{1}\right\rangle \text { is <o-increasing, }\left|\mathbb{P}_{i}\right| \leq \aleph_{1},\right. \\
& \underset{\sim}{\bar{r}}=\left\langle\underset{\sim}{r_{i}}: i<\omega_{1}\right\rangle,{\underset{\sim}{r}}_{i} \text { is a } \mathbb{P}_{i+1} \text {-name, } \\
& \left.\left.\Vdash_{\mathbb{P}_{i+1}} \stackrel{\sim}{r} i_{i} \in{ }^{\omega} \omega \text { dominates }\left({ }^{\omega} \omega\right) \mathbf{V}^{\mathbb{P}_{i}}\right] "\right\}
\end{aligned}
$$

ordered naturally, and for a generic enough $\omega_{2}$-limit $\left\langle\left(\overline{\mathbb{P}}^{\zeta}, \bar{\sim}^{\zeta}\right): \zeta<\omega_{2}\right\rangle$ we may use $\bigcup_{i<\omega_{1}, \zeta<\omega_{2}} \mathbb{P}_{i}^{\zeta}$. Another way to try is the non-Cohen Oracle [116]. The difference is small. Also the " $\omega_{2}+\omega_{1}$-length mix finite/countable pure support iteration" seems similar.

I have just heard about $\operatorname{CON}(\mathfrak{u}<\mathfrak{a})$ being an old problem, clearly related to $\operatorname{CON}(\mathfrak{d}<\mathfrak{a})$. I do not see much difference at present.

Another direction is to develop the historic $\aleph_{\varepsilon}$-support iteration from [109].

Discussion 3.3. Concerning 2.9, I had not really considered it (except when Judah spoke to me about it) but just before the lecture, Bartoszyński reminded me of it (see [7]). Now, "the" proof of CON(Borel conjecture) is by CS iteration of Laver forcing (see Laver [76]), whereas the consistency proof of the dual is adding many Cohen reals (see Carlson [19]). So in a (hopeful) iteration proving consistency we have two kinds of assignments. We are given, say in stage $\alpha$, in $\mathbf{V}^{\mathbb{P}_{\alpha}}$ a set $A=\left\{\eta_{i}: i<\omega_{1}\right\} \subseteq{ }^{\omega}{ }_{2}$, and we should make it not of strong measure zero, so we should add an increasing sequence $\bar{n}=\left\langle n_{l}: l<\omega\right\rangle$ of natural numbers such that for no $\bar{\nu}=\left\langle\nu_{l}: l<\omega\right\rangle \in \prod_{l<\omega}{ }^{\left(n_{l}\right)} 2$ do we have $\left(\forall i<\omega_{1}\right)\left(\exists^{\infty} l\right)\left(\nu_{l} \triangleleft \eta_{i}\right)$. Now, even if we define $\mathbb{Q}_{\alpha}$ to add such $\bar{n}$, we have to preserve it later, so it is 
easier to preserve, for some family $F \subseteq \prod_{l<\omega} 2^{n_{l}}$, the demand

$$
(\forall f \in F) \neg\left(\exists\left\langle\nu_{l, k}: l<\omega, k<f(l)\right\rangle\right)\left(\forall i<\omega_{1}\right)\left(\exists^{\infty} l\right)(\exists k<f(l))\left(\nu_{l, k} \triangleleft \eta_{i}\right) .
$$

The second kind of assignment which we may have in stage $\alpha$ is the following. In $\mathbf{V}^{\mathbb{P}_{\alpha}}$, we are given $A=\left\{\eta_{i}: i<\omega_{1}\right\} \subseteq{ }^{\omega} 2$ and we should make it nonstrongly meagre, so we should add, by $\mathbb{Q}_{\alpha}$, a subtree $T_{\alpha} \subseteq{ }^{\omega>} 2$ (i.e., \langle\rangle$\in T_{\alpha}$, $\left.\eta \in T_{\alpha} \& \nu \triangleleft \eta \Rightarrow \nu \in T_{\alpha}, \eta \in T_{\alpha} \Rightarrow(\exists l<2)\left(\eta \smile\langle l\rangle \in T_{\alpha}\right)\right)$ of positive measure (i.e., $\left.0<\inf \left\{\left|T_{\alpha} \cap{ }^{n} 2\right| / 2^{n}: n<\omega\right\}\right)$ such that $\left(\forall \eta \in{ }^{\omega} 2\right)(\exists i<$ $\left.\omega_{1}\right)\left[\eta \oplus \eta_{i} \notin \bigcup_{n}\left(T_{\alpha}\right)^{[n]}\right]$, where

$$
\begin{array}{r}
\left(T_{\alpha}\right)^{[n]}=\left\{\nu: \text { for some } \varrho \in T_{\alpha} \text { we have } \lg (\nu)=\lg (\varrho)\right. \\
\text { and }(\forall l)(n \leq l<\lg (\nu) \Rightarrow \varrho(l)=\nu(l))\} .
\end{array}
$$

Again we have to preserve this.

A way to deal with such preservation problems is to generalize "oracle c.c.c." (see [176, Ch. IV]) replacing Cohen by other things. To explain this, it seems reasonable to look at the "oracle for random" (or even sequence of c.c.c. Suslin forcing, from [116]). This evolves to: for iterations of length $\leq \omega_{2}$ of forcing notions of cardinality $\aleph_{1}$, prove that we can preserve the following condition on $\mathbb{P}=\mathbb{P}_{\alpha}$ for some $\left\langle M_{\delta}, M_{\delta}^{+}, r_{\delta}: \delta \in S\right\rangle, S \subseteq \omega_{1}$ stationary such that $\left\langle M_{\delta}: \delta \in S\right\rangle$ is an oracle, i.e., a $\diamond^{*}$-sequence and $M_{\delta} \models \delta=\omega_{1}, M_{\delta}=\mathrm{ZFC}_{*}^{-}, M_{\delta}^{+} \models " \mathrm{ZFC}_{*}^{-}+M_{\delta}$ is countable" and $r_{\delta}$ is random over $M_{\delta}^{+}$. Now without loss of generality, $\mathbb{P} \subseteq \omega_{1}$ and

$$
\begin{aligned}
& \left\{\delta \in S: \mathbb{P}\left\lceil\delta \in M_{\delta} \text {, and for every } p \in \mathbb{P} \cap \delta,\right.\right. \\
& \text { for some } q \text { we have } p \leq q \in \mathbb{P} \text { and } \\
& \left.q \Vdash \text { " } r_{\delta} \text { is random over } M_{\delta}^{+}\left[G_{\mathbb{P}} \cap \delta\right] "\right\} \in \mathcal{D}_{\omega_{1}}\lceil S
\end{aligned}
$$

(so this is like the oracle c.c.c. ([176, Ch. IV]), but the support is not countable so on other stationary $S_{1} \subseteq \omega_{1} \backslash S$ we may have different behaviour). Of course, we use "small" $S$ so that we have "space" for more demands, see [116]. But trying to explain it (to Rosłanowski) it seemed the proof is too simple, so we can go back to good old CS and just preserving an appropriate property, a watered-down relative in the nep family ([115]).

We mainly try to combine the two iterations (of Cohen and of Laver forcing notions):

Definition 3.4. A forcing notion $\mathbb{Q}$ is 1-e.l.c. if the following condition is satisfied: whenever $\chi$ is large enough, $M_{0} \prec M_{1} \prec(\mathcal{H}(\chi), \in), \mathbb{Q} \in M_{0}$, $M_{0} \in M_{1}$ and $M_{0}, M_{1}$ are countable and $p \in \mathbb{Q} \cap M_{0}$, then for some condition $q \in \mathbb{Q}$ stronger than $p$ we have

$q \Vdash$ "for every $\mathcal{I} \in M_{1}$ such that $\mathcal{I} \cap M_{0}$ is predense in $\mathbb{Q}^{M_{0}}$ we have $G_{\mathbb{Q}} \cap \mathcal{I} \neq \emptyset "$. 
(Note that $q \Vdash " M_{0}\left[G_{\mathbb{Q}} \cap M_{0}\right]$ is a generic extension of $M_{0}$ for a forcing notion which $M_{1}$ thinks is countable".)

Note: e.l.c. stands for elementary locally Cohen. This is, of course, close to Cohen, or more accurately is another way to present strongly proper. But we also seem to need Laver forcing (or a close relative of it), but it is far from being strongly proper. Still it satisfies the parallel if we demand " $\mathcal{I} \subseteq Q^{M_{1}}$ is predense under pure extensions", i.e., with the same trunk. This approach seems to me promising but it is not clear what it delivers.

We may consider a more general definition (and natural preservation):

Definition 3.5. Let Pr be a property. A forcing notion $\mathbb{Q}$ with generic $\underset{\sim}{X} \subseteq \alpha_{\mathbb{Q}}$ (i.e. $\mathbf{V}\left[G_{\mathbb{Q}}\right]=\mathbf{V}\left[\underset{\sim}{X}\left[G_{\mathbb{Q}}\right]\right], \alpha_{\mathbb{Q}}$ an ordinal) is called an e.l.-Pr forcing if for $\chi$ large enough, if $\mathbb{Q}, \underset{\sim}{X} \in M_{1} \prec M_{2} \prec(\mathcal{H}(\chi), \in), M_{1}, M_{2}$ countable, $M_{1} \in M_{2}, p \in \mathbb{Q} \cap M_{1}$, then we can find $q, \mathbb{Q}^{\prime}$ such that

(a) $p \leq q \in \mathbb{Q}$,

(b) $\mathbb{Q}^{\prime} \in M_{2}$ is a forcing notion with $\underset{\sim}{X} \subseteq \alpha_{\mathbb{Q}}$ generic,

(c) $M_{2} \models \operatorname{Pr}\left(\mathbb{Q}^{\prime}, M_{1}, p\right)$,

(d) $q \Vdash “ \underset{\sim}{X}\left\lceil M_{2}\right.$ is a $\mathbb{Q}^{\prime}$-generic over $p$ and for some set $G^{\prime} \subseteq\left(\mathbb{Q}^{\prime}\right)^{M_{2}}$, generic over $M_{2}$ we have $\underset{\sim}{X}\left\lceil M_{2}=\underset{\sim}{X}\left[G^{\prime}\right]\right.$ ".

This seems to me interesting but though Laver forcing satisfies some relatives of those properties it does not seem to be enough.

Note: this definition tells us that generically for many countable models $M \prec(\mathcal{H}(\chi), \in)$, we have some $q \in G_{\mathbb{Q}}$ which is almost $(M, \mathbb{Q})$-generic, but not quite. The "almost" is because this holds for another forcing $\mathbb{Q}^{\prime}$. So when the whole universe is extended generically for $\mathbb{Q}, M$ "fakes" and is instead extended generically for $\mathbb{Q}^{\prime}$. So for preservation in iteration it is not natural to demand $M_{2} \prec(\mathcal{H}(\chi), \in)$, but rather to proceed as in [115], this will be n.e.l.-Pr.

We may wonder (considering 2.9) whether we can replace Laver forcing in the proof of the consistency of the Borel conjecture by a forcing notion not adding a dominating real. So a question related to 2.9 is

Question 3.6. $\operatorname{CON}\left(\mathfrak{b}=\aleph_{1}+\right.$ Borel Conjecture $) ?$

It is most natural to iterate, one basic step will be $\mathbb{Q}$, adding an increasing sequence $\left\langle\underset{\sim}{n}{ }_{i}: i<\omega\right\rangle$ such that on the one hand:

(a) no old non-dominated family $\subseteq{ }^{\omega} \omega$ is dominated (or at least some particular old family remains undominated), while on the other hand

(b) for any uncountable $A \subseteq{ }^{\omega} 2$, from $\mathbf{V}$, we have:

$\Vdash_{\mathbb{Q}}$ "for no $\eta_{\sim} \in{ }^{n_{i}} 2,(i<\omega)$ do we have $(\forall \nu \in A)\left(\exists^{\infty} i\right)\left(\eta_{\sim} \triangleleft \nu\right)$ ", or at least 
(b)' like (b) for one $A$ given by bookkeeping.

(To preserve we need to strengthen the statement, replacing $\left\langle\eta_{i}: i<\omega\right\rangle$ by a thin enough tree.) The $\eta_{i}$ should "grow" fast enough, so naturally we think of forcing notions as in Rosłanowski and Shelah [92], [88], which proved easily checked sufficient conditions for what we desire. It is natural to look for forcing notions in the "neighborhood" of Blass and Shelah [15]. But what should be the norm?

Discussion 3.7. Concerning $\mathfrak{p}<\mathfrak{t}$, I have made quite a few failed tries. Some try to use long iterations $\left(\geq \aleph_{\omega+1}\right)$ or a new support. But also I thought that Blass and Shelah [16] would be a reasonable starting point; the point is how to extend $\aleph_{1}$-generated filters to a good enough $P$-point.

That is, trying to force $\mathfrak{p}=\aleph_{2}, \mathfrak{t}=\aleph_{3}=\mathfrak{c}$ start, say, with $\mathbf{V}=\mathbf{L}$ and use a FS iteration $\left\langle\mathbb{P}_{i}, \mathbb{Q}_{j}: i \leq \omega_{3}, j<\omega_{3}\right\rangle$, where $\mathbb{Q}_{i}$ is a Cohen forcing adding $\underline{r}_{i} \in{ }^{\omega} 2$ for some $i$ 's, and $\mathbb{Q}_{i}$ is shooting an $\tilde{\omega}$-sequence through a $P$-point filter (or ultrafilter) on $\omega$ for some $i \geq \omega_{2}$. The point is that when we have to find a $\leq^{*}$-lower bound to the downward directed $\mathcal{A} \in[\mathcal{P}(\omega)]^{\aleph_{1}}$, we extend it to a $P$-point; possibly also we have to do this for the $\omega_{2}$-towers. It is natural to try to preserve, for $\alpha \in\left[\omega_{2}, \omega_{3}\right)$, the statement:

- in $\mathbf{V}^{\mathbb{P}_{\alpha}}$, noting that $\mathcal{H}\left(\aleph_{1}\right)$ has cardinality $\aleph_{2}$, if $\mathcal{H}\left(\aleph_{1}\right)=\bigcup_{\alpha<\omega_{2}} M_{\alpha}$, $M_{\alpha}$ increasing continuous, $\left\|M_{\alpha}\right\|<\aleph_{2}$, then the following set is = $\emptyset \bmod \mathcal{D}_{\omega_{2}}+S_{1}^{2}$ :

$\left\{\delta\right.$ : if some $a \in M_{\delta} \cap[\omega]^{\aleph_{0}}$ is almost included in $r_{i}^{-1}(\{1\})$

for many $i<\delta$, then $a$ is almost disjoint from $\left.r_{\delta}^{-1}(\{1\})\right\}$.

Discussion 3.8. Concerning 2.11 consider the problem "every $f: \mathbb{R} \rightarrow \mathbb{R}$ is continuous on a non-null set".

We can try to use a forcing notion which looks locally random (like the forcing for "non-meagre set" of [169] looked locally like the Cohen forcing notion) or a mixture of random and quite bounding ones. Such forcing notions are considered in [88]; do they help for "every function $f:{ }^{\omega} 2 \rightarrow{ }^{\omega} 2$ is continuous on a non-null set"?

How can we try to prove the consistency of "for every non-meagre $A \subseteq$ $\omega_{2}$ and $f:{ }^{\omega_{2}} \rightarrow{ }^{\omega_{2}}$ for some non-meagre $B \subseteq A, f\lceil B$ is continuous"? We may use CS or even FS iteration of length $\omega_{2}$, (with $\mathbf{V} \models$ GCH + $\diamond_{\left\{\delta<\aleph_{2}: \operatorname{cf}(\delta)=\aleph_{1}\right\}}+S_{\alpha} \subseteq \omega_{1}\left(\alpha<\omega_{2}\right)$ increasing $\left.\bmod \mathcal{D}_{\omega_{1}}\right)$. 
In stage $\alpha$ we have $\bar{r}^{\alpha}=\left\langle\sim_{i}^{\alpha}: i \in S_{\alpha}\right\rangle$ such that $\bar{r}^{\alpha} \in N \prec(\mathcal{H}(\chi), \in)$ $\Rightarrow \underset{\sim}{r} \alpha \cap \omega_{1}$ is forced to be Cohen over $N$ and $\beta<\alpha \Rightarrow\left\{i \in S_{\beta}:{\underset{\sim}{r}}_{i}^{\beta} \neq{\underset{\sim}{r}}_{i}^{\alpha}\right\}$ is not stationary.

Sometimes in stage $\alpha$, bookkeeping gives us a $\mathbb{P}_{\alpha}$-name $\underset{\sim}{A_{\alpha}}$ of a nonmeagre subset of $\omega_{2}$ and we choose $\bar{r}^{\alpha+1}$ such that $\bar{r}^{\alpha+1} \uparrow \tilde{S}_{\alpha}=\bar{r}^{\alpha}$ and $\left\{r_{i}^{\alpha+1}: i \in S_{\alpha+1} \backslash S_{\alpha}\right\}$ is a non-meagre subset of $\underset{\sim}{A}$.

Sometimes in stage $\alpha$, bookkeeping gives us a stationary subset $S^{\alpha}$ of $S_{\alpha}$ (from $\mathbf{V}$ ) and a $\mathbb{P}_{\alpha}$-name $f_{\alpha}$ of a function from $\left\{{\underset{\sim}{i}}_{i}^{\alpha}: i \in S^{\alpha}\right\}$ to ${ }^{\omega} 2$ and we try to choose $\bar{r}^{\alpha+1}$ such that $\bar{r}^{\alpha+1} \uparrow S_{\alpha}=\bar{r}^{\alpha},\left\{\sim_{i}^{\alpha+1}: i \in S_{\alpha+1} \backslash S_{\alpha}\right\} \subseteq$ $\left\{\stackrel{r}{i}_{i}^{\alpha}: i \in S^{\alpha}\right\}$ and $f_{\alpha}\left\lceil\left\{\underline{r}_{i}^{\alpha+1}: i \in S_{\alpha+1} \backslash S_{\alpha}\right\}\right.$ is continuous. So the aim is that in $\mathbf{V}^{\mathbb{P}_{\omega_{2}}}$, every non-meagre $A \subseteq{ }^{\omega_{2}} 2$ contains a subset of the form $\left\{\sim_{i}^{\alpha}: i \in S_{\alpha+1} \backslash S_{\alpha}\right\}$ and

$S^{\prime} \subseteq S_{\alpha} \& \alpha<\omega_{1} \& S^{\prime}$ stationary $\Rightarrow\left\{r_{i}^{\alpha}: i \in S^{\prime}\right\}$ is non-meagre.

We may try to define iterations for forcing related to measure: we can use CS, or try to imitate the measure algebra, there are various ways to interpret it. If each $\mathbb{Q}_{i}$ is as in [92], then each condition has a possible pair: a norm $\in \omega$ and real $r \in(0,1)$ and using those we define what is a condition in the iteration. See [88].

Remember that an automorphism $F$ of $\mathcal{P}(\omega)$ /finite is called trivial if it is induced by a permutation $f$ of $\mathbb{Z}$ (the integers, where $\omega=\{n \in \mathbb{Z}: n \geq 0\}$ ) such that $\{n \in \mathbb{Z}: n<0 \Leftrightarrow f(n) \geq 0\}$ is finite.

Question 3.9. What can $\operatorname{AUT}(\mathcal{P}(\omega) /$ finite) be? Is it consistent that $\operatorname{AUT}(\mathcal{P}(\omega)$ /finite) is not the group of trivial automorphisms of $\mathcal{P}(\omega)$ /finite, but is of cardinality continuum (or even is generated by adding one automorphism to the subgroup of the trivial automorphisms of $\mathcal{P}(\omega) /$ finite $)$ ?

It is reasonable to try to combine Shelah and Steprāns [184] and the latter part of the proof in $[176, \mathrm{Ch}$. IV, §6] (from being "locally trivial" to being trivial).

Discussion 3.10. Next we deal with the variants of OCA and isomorphisms or farness of sets of $\aleph_{2}$ reals, i.e. $2.21,2.24$.

Concerning 2.21 (on "there are no far $A, B \in\left[{ }^{\omega} 2\right]^{\lambda}$ "), assume that for $l=1,2$ we have $A_{l}=\left\{\eta_{\alpha}^{l}: \alpha<\lambda\right\} \subseteq{ }^{\omega} 2$ with no repetitions. Considering Baumgartner [10] and Abraham, Rubin and Shelah [2], it is natural to try to find $\bar{f}=\left\langle f_{\alpha}: \alpha<\lambda\right\rangle$ such that:

(a) $f_{\alpha}$ is a partial, countable, non-empty function from $\omega_{2}$ to $\omega_{2}$ (for the present aim, $\operatorname{Dom}\left(f_{\alpha}\right)$ a singleton in (a) and $\gamma=1$ in (b) are fine, so we assume so), 
(b) for some $\gamma=\gamma^{*} \leq \omega$, the sequence

$$
\left\langle\bigcup_{n<\gamma}\left(\operatorname{Dom}\left(f_{\gamma \alpha+n}\right) \cup \operatorname{Rang}\left(f_{\gamma \alpha+n}\right)\right): \alpha<\lambda\right\rangle
$$

is a sequence of pairwise disjoint sets.

We let $\hat{f}_{\alpha}=\left\{\left(\eta_{i}^{1}, \eta_{f_{\alpha}(i)}^{2}\right): i \in \operatorname{Dom}\left(f_{\alpha}\right)\right\}$, so $\hat{f}_{\alpha}(\eta)$ is well defined iff $i \in \operatorname{Dom}\left(f_{\alpha}\right), \eta=\eta_{i}^{1}$, and then $\hat{f}_{\alpha}\left(\eta_{i}^{1}\right)=\eta_{f_{\alpha}(i)}^{2}$.

It is natural to try the following forcing notion:

$\mathbb{Q}_{\bar{f}}=\left\{g: g\right.$ is a finite 1 -to-1 order preserving function from $A_{1}$ to $A_{2}$ which has the form $\bigcup_{l=1}^{n} f_{\alpha_{l}}$, where $\alpha_{l}<\omega_{2}$, $\left.l_{1} \neq l_{2} \Rightarrow \neg(\exists \alpha)\left(\left\{\alpha_{l_{1}}, \alpha_{l_{2}}\right\} \subseteq\left[\gamma^{*} \alpha, \gamma^{*} \alpha+\gamma^{*}\right]\right)\right\}$.

The order is the inclusion.

It is enough to have " $\mathbb{Q}_{\bar{f}}$ satisfies the c.c.c." (for some $\bar{f}$ as above): clearly $\bigcup\left\{g: g \in G_{\mathbb{Q}_{\bar{f}}}\right\}$ is an order preserving function from some $A_{1}^{\prime} \subseteq A_{1}$ into $A_{2}$; but does it have cardinality $\lambda$ ? Essentially yes, as e.g. if $\operatorname{cf}(\lambda)>\aleph_{0}$ then some $p \in \mathbb{Q}_{\bar{f}}$ forces this. For this it is enough to have: if $u \in[\lambda]^{\aleph_{1}}$ then $\mathbb{Q}_{\bar{f}\lceil u}$ satisfies the c.c.c.; when $\lambda=\aleph_{2}$ it is enough to check for $u=\alpha \in$ $\left[\omega_{1}, \omega_{2}\right)$. So as in $[2]$, it is enough that

(c) if $n<\omega$ and $C \subseteq{ }^{2 n}\left(\omega_{2}\right)$ is closed and $\left(\eta_{0}, \eta_{1}, \ldots, \eta_{2 n-1}\right) \in C \Rightarrow(\forall l<m<n)\left(\eta_{2 l}<\right.$ lex $\eta_{2 m} \Leftrightarrow \eta_{2 l+1}<$ lex $\left.\eta_{2 m+1}\right)$ and there are $p_{\zeta}=\left\{\left(\eta_{\zeta, l}, \nu_{\zeta, l}\right): l \leq n\right\} \in \mathbb{Q}_{\bar{f}}$ for $\zeta<\omega_{1}, l<n$ with

$$
\left(\zeta_{1}, l_{1}\right) \neq\left(\zeta_{2}, l_{2}\right) \Rightarrow \eta_{\zeta_{1}, l_{1}} \neq \eta_{\zeta_{2}, l_{2}} \text { and } \nu_{\zeta_{1}, l_{1}} \neq \nu_{\zeta_{2}, l_{2}}
$$

hence

$$
\left(\forall \zeta<\omega_{1}\right)(\forall l<m<n)\left(\eta_{\zeta, l}<_{\operatorname{lex}} \eta_{\zeta, m} \Leftrightarrow \nu_{\zeta, l}<_{\text {lex }} \nu_{\zeta, m}\right)
$$

(if $\gamma^{*}=1$, and each $\operatorname{Dom}\left(f_{\alpha}\right)$ is a singleton, $p_{\zeta} \in \mathbb{Q}_{\bar{f}}$ means in addition only $\left\{\left(\eta_{\zeta, l}, \nu_{\zeta, l}\right)\right\}=f_{\alpha_{\zeta, l}}$ for some $\left.\alpha_{\zeta, l}\right)$, then there are $\left(\eta_{0}^{\prime}, \eta_{1}^{\prime}, \ldots, \eta_{2 n-1}^{\prime}\right),\left(\eta_{0}^{\prime \prime}, \eta_{1}^{\prime \prime}\right.$, $\left.\ldots, \eta_{2 n-1}^{\prime \prime}\right) \in C$ such that $\eta_{l}^{\prime} \neq \eta_{l}^{\prime \prime}$ and $\eta_{2 l}^{\prime}<_{\text {lex }} \eta_{2 l}^{\prime \prime} \Leftrightarrow \eta_{2 l+1}^{\prime}<_{\text {lex }} \eta_{2 l}^{\prime \prime}$.

Any counterexample to clause (c) induces a continuous partial function for which we get dependencies. If $\mathbf{V}=\mathrm{CH}$, and $\mathbb{P}$ is adding Cohen reals, then in $\mathbf{V}^{\mathbb{P}}$ this holds, so it is natural to try to retain it during the iteration similarly to this case. For 2.20 we can use a similar, but somewhat more involved forcing notion as in [2].

There are similar considerations on OCA. We may consider trying to get negative ZFC results, so Kojman and Shelah [70] seems to me a reasonable starting point (of course, the problem there is different). 
Baumgartner [12] defines

Definition 3.11. (1) For a (non-principal) ultrafilter $D$ on $\omega$, and a countable ordinal $\delta$, we say $D$ is a $\delta$-ultrafilter if:

(*) for every function $f$ from $\omega$ to $\omega_{1}$, for some $A \in D$ we have $\operatorname{otp}(f(A))$ $<\delta$.

(2) We say that $D$ is a weak $\delta$-ultrafilter if:

$(*)^{-} \quad$ for every function $f$ from $\omega$ into $\delta$, for some $A \in D$ we have $\operatorname{otp}(f(A))$ $<\delta$.

(3) We say that $D$ is a $N W D$ if for every function $f$ from $\omega$ to $\mathbb{R}$, for some $A \in D, f(A)$ is a nowhere dense subset of $\mathbb{R}$.

(4) For an ideal $I, D$ is an $I$-filter if for any $f: \operatorname{Dom}(D) \rightarrow \operatorname{Dom}(I)$ and $A \in D^{+}$there is $B \subseteq A, B \in D^{+}$such that $f(B) \in I$.

Then he asked whether such ultrafilters exist (if $\mathrm{CH}$ yes, so):

QUESTION 3.12. Prove the consistency of "there is no $\delta$-ultrafilter on $\omega "$.

It seemed the solution of the related "CON(there is no NWD-ultrafilter)" in [177] should give this, but it did not, and it is not clear if the question 3.12 is harder (the NWD eluded me several times, but when solved, the solution seems a straightforward generalization of $\mathrm{CON}$ (no $P$-point), which was also a priori the natural starting point).

A nice feature of $P$-points is that " $D$ generates a $P$-point ultrafilter on $\omega$ " is preserved in limit for CS iterations, so $P$-points generated by $\aleph_{1}<2^{\aleph_{0}}$ sets are obtained naturally. Are they the only ones? Of course, by c.c.c. forcing $P$ you may have ultrafilters on $\omega$ generated by $<2^{\aleph_{0}}$ sets, and forcing by a subforcing $\mathbb{Q} \prec \mathbb{P}$, in $\mathbf{V}^{\mathbb{Q}}$ we get an ultrafilter preserved (see more in Brendle and Shelah [18]); but we have no understanding, though the suggestion in 3.2 may help.

Question 3.13. Are ultrafilters $D$ as defined below in 3.21 preserved in limit stages of CS iterations? This means that: if $\overline{\mathbb{Q}}=\left\langle\mathbb{P}_{i}, \mathbb{Q}_{j}: i \leq \delta, j<\delta\right\rangle$ is a CS iteration of proper forcing notions and $D$ is an ultrafilter as above in $\mathbf{V}$, then $(\forall i<\delta)\left(\left(\bigotimes_{i}\right) \Rightarrow\left(\bigotimes_{\delta}\right)\right)$, where

$\left(\otimes_{\beta}\right) \quad \Vdash_{\mathbb{P}_{\beta}}$ "in $\mathbf{V}^{\mathbb{P}_{\beta}}$ the filter on $\omega$ that the family $D$ generates in $\mathbf{V}^{\mathbb{P}_{\beta}}$ is an ultrafilter".

Less nice, but still good, is to prove the preservation of " $D$ generates an ultrafilter $+\operatorname{Pr} "$ (where Pr is some additional property like: $\left({ }^{\omega} \omega\right)^{\mathbf{V}}$ is dominating)

The following 3.14-3.22 suggest an approach to Question 3.13. 
Definition 3.14. (1) Let

$\mathcal{T}=\left\{t: t \subseteq{ }^{\omega>} \omega, t\right.$ has a $\triangleleft$-minimal element $\operatorname{rt}(t)$,

$t$ is closed under initial segments of length $\geq \lg (\mathrm{rt}(t))$,

for $\eta \in t, \operatorname{Suc}_{t}(\eta)=\{\eta \frown\langle l\rangle: \eta \frown\langle l\rangle \in t\}$ is empty or infinite $\}$.

For $t \in \mathcal{T}$ let $h_{t}: t \rightarrow \omega_{1} \cup\{\infty\}$ be defined by

$$
h_{t}(\eta)=\bigcup\left\{h_{t}(\nu)+1: \nu \in \operatorname{Suc}_{t}(\eta)\right\} .
$$

(So $h_{t}(\eta)=\infty$ iff there is an $\omega$-branch through $\eta$.) For $\eta \in t \in \mathcal{T}$ let $t^{[\eta]}=\{\nu \in t: \eta \unlhd \nu\}$. We say that $t$ is standard if

$$
\eta \in t \& \beta<h_{t}(\eta) \Rightarrow\left(\forall^{\infty} \nu \in \operatorname{Suc}_{t}(\eta)\right)\left(\beta \leq h_{t}(\nu)\right) .
$$

If not said otherwise, every $t$ is standard. Note:

$$
s \in \operatorname{sub}(t) \& t \text { is standard } \Rightarrow s \text { is standard, }
$$

where on $\operatorname{sub}(t)$ see part (8) below.

(2) For an ordinal $\alpha<\omega_{1}$, let

$$
\mathcal{T}_{\alpha}=\left\{t \in \mathcal{T}: \operatorname{Rang}\left(h_{t}\right) \subseteq \omega_{1} \text { and } h_{t}(\operatorname{rt}(t))=\alpha\right\}, \quad \mathcal{T}_{<\alpha}=\bigcup_{\beta<\alpha} \mathcal{T}_{\beta} .
$$

(3) For $t \in \mathcal{T}$ let

$\mathcal{A}_{t}=\left\{\bar{A}: \bar{A}=\left\langle A_{\eta}: \eta \in t\right\rangle, A_{\eta} \in[\omega]^{\aleph_{0}}\right.$, and if $\eta \in t$ is not maximal, then $\left\langle A_{\nu}: \nu \in \operatorname{Suc}_{T}(\eta)\right\rangle$ is a sequence of pairwise disjoint subsets of $\left.A_{\eta}\right\}$,

$$
\mathcal{A}_{\alpha}=\bigcup\left\{\mathcal{A}_{t}: t \in \mathcal{T}_{\alpha}\right\}, \quad \mathcal{A}_{<\alpha}=\bigcup_{\beta<\alpha} \mathcal{A}_{\beta} .
$$

(4) For $t \in \mathcal{T}$ and $\eta \in t$ let $t^{[\eta]}=\{\nu: \eta \unlhd \nu \in t\} \in \mathcal{T}$.

(5) For $t \in \mathcal{T} \operatorname{let} \lim (t)=\left\{\eta \in{ }^{\omega} \omega:(\forall l<\omega)(l \geq \lg (\operatorname{rt}(t)) \Rightarrow \eta\lceil l \in t)\}\right.$.

(6) $\max (t)=\left\{\eta \in t: \operatorname{Suc}_{t}(\eta)=\emptyset\right\}$.

(7) We say that $y$ is a front of $t \in \mathcal{T}$ if $y \subseteq t,(\forall \eta, \nu \in y)(\neg \eta \triangleleft \nu)$ and

$$
(\forall \eta)[(\eta \in \lim (t) \vee \eta \in \max (t)) \Rightarrow(\exists l \leq \lg (\eta))(\eta\lceil l \in y)] .
$$

We let $\operatorname{fr}(t)=\{y: y$ is a front of $t\}$.

(8) For $t \in \mathcal{T}$ let $\operatorname{sub}(t)=\{s \in \mathcal{T}: s \subseteq t$ and $\max (s)=\max (t) \cap s\}$. Clearly for standard $t$ and $s, s \in \operatorname{sub}(t)$, we have $h_{s}=h_{t}\lceil s$. Let

$$
\operatorname{sub}^{-}(t)=\{s \in \mathcal{T}: s \subseteq t, s \in \operatorname{sub}(t) \text { and } \operatorname{rt}(s)=\operatorname{rt}(t)\} .
$$

(9) For $s, t \in \mathcal{T}$ and a set $\mathcal{Y}$ of fronts of $t$ let $\mathrm{FT}_{\mathcal{Y}}^{0}(t, s)$ be the set of projections $f: s \rightarrow t$ (i.e., $f$ is a function, $\operatorname{Dom}(f) \subseteq s, \operatorname{Rang}(f) \subseteq t$, $f(\operatorname{rt}(s))=\operatorname{rt}(t)$ and $(\forall \eta, \nu \in t)(\eta \triangleleft \nu \Rightarrow f(\eta) \triangleleft f(\nu)))$ which respect each $y \in \mathcal{Y}$, i.e., $\{\eta \in \operatorname{Dom}(f): f(\eta) \in y\}$ is a front of $s$ for every $y \in \mathcal{Y}$. 
(10) If $\mathcal{Y}$ is the set of all fronts of $t$ we may omit it.

(11) $\mathrm{FT}^{1}(t, s)$ is the set of $f \in \mathrm{FT}^{0}(t, s)$ such that

$$
\eta \in s \Rightarrow h_{s}(\eta)=h_{t}(f(\eta)) .
$$

(12) $\mathrm{FT}^{2}(t, s)$ is the set of all partial functions $f$ from $s$ onto $t$ such that:

- $\eta \triangleleft \nu \Leftrightarrow f(\eta) \triangleleft f(\nu)$

- $\left\{f^{-1}(\eta): \eta \in \operatorname{Suc}_{s}(\operatorname{rt}(s))\right\}$ is a front of $t$,

- for $\eta \in \operatorname{Suc}_{s}(\operatorname{rt}(s)), f\left\lceil s^{[\eta]}\right.$ is one-to-one onto $t^{[f(\eta)]}$.

$\mathrm{FT}^{3}(t, s)$ is the set of all $f: s \rightarrow t$ such that:

- $\operatorname{Dom}(f)=s, \max (t) \cap \operatorname{Rang}(f)=\{f(\eta): \eta \in \max (s)\}$,

- $\eta \triangleleft \nu \Leftrightarrow f(\eta) \triangleleft f(\nu)$, for $\eta, \nu \in s$,

- $\eta \in s \& \nu \in \operatorname{Suc}_{t}(\eta) \Rightarrow f(\nu) \in \operatorname{Suc}_{t}(f(\eta))$.

(13) For an ideal $I$ let

$\mathcal{S} \mathcal{T}=\mathcal{S T}_{I}=\{(t, g): t \in \mathcal{T}$ and $g: t \backslash\{\operatorname{rt}(t)\} \rightarrow \operatorname{Dom}(I)$ are such that

$$
\left.\eta \in t \backslash \max (t) \Rightarrow\left\{g(\nu): \nu \in \operatorname{Suc}_{t}(\eta)\right\} \in I^{+}\right\}
$$

We usually omit $I$ if it is clear from the context (here it is fixed).

(14) For $\left(t^{1}, g^{1}\right),\left(t^{2}, g^{2}\right) \in \mathcal{S} \mathcal{T}$ for $l=0,1,2$ let $\mathcal{F} \mathcal{T}^{l}\left(\left(t^{1}, g^{1}\right),\left(t^{2}, g^{2}\right)\right)$ be the set of $f \in \mathrm{FT}^{l}\left(t^{1}, t^{2}\right)$ such that $g^{2}=g^{1} \circ f$.

(15) Let

$$
\begin{array}{rc}
\mathcal{T} \mathcal{T}_{t}=\left\{(t, \bar{A}, g): \bar{A} \in \mathcal{A}_{t} \text { and }(t, g) \in \mathcal{S} \mathcal{T}\right\}, & \mathcal{T} \mathcal{T}=\bigcup_{t} \mathcal{T} \mathcal{T}_{t} \\
\mathcal{T} \mathcal{T}_{\alpha}=\left\{(t, \bar{A}, g): t \in \mathcal{T}_{\alpha} \text { and }(t, \bar{A}, g) \in \mathcal{T} \mathcal{T}_{t}\right\}, & \mathcal{T} \mathcal{T}_{<\alpha}=\bigcup_{\beta<\alpha} \mathcal{T} \mathcal{T}_{\beta}
\end{array}
$$

(16) Let $\mathcal{F}^{l}\left(\left(t^{1}, \bar{A}^{1}, g^{1}\right),\left(t^{2}, \bar{A}^{2}, g^{2}\right)\right)$ be the set of $f \in \mathcal{F}^{l}\left(\left(t^{1}, g^{1}\right)\right.$, $\left.\left(t^{2}, g^{2}\right)\right)$ such that $\eta \in t^{2} \Rightarrow A_{f(\eta)}^{1} \subseteq^{*} A_{\eta}^{2}$.

(17) If we omit $l$ (in $\mathrm{FT}^{l}, \mathcal{F} \mathcal{T}^{l}$ ) we mean $l=0$.

Definition 3.15. We define a partial order on $\mathcal{T} \mathcal{T}_{<\alpha}$ : $\left(t^{1}, \bar{A}^{1}, g^{1}\right) \leq^{l}\left(t^{2}, \bar{A}^{2}, g^{2}\right)$ if and only if $\mathcal{F} \mathcal{T}^{l}\left(\left(t^{1}, \bar{A}^{1}, g^{1}\right),\left(t^{2}, \bar{A}^{2}, g^{2}\right)\right) \neq \emptyset$.

OBSERVATION 3.16. (1) $\leq^{l}$ really is a partial order on $\mathcal{T} \mathcal{T}$.

(2) $\leq^{2} \subseteq \leq^{1} \subseteq \leq^{0}$ and $\leq^{3} \subseteq \leq^{0}$ and lastly among standard $t$ 's we have $\leq^{3} \subseteq \leq^{1}$.

(3) For all $\left(t^{1}, \bar{A}^{1}, g^{1}\right) \in \mathcal{T} \mathcal{T}$ there is a standard $\left(t^{2}, \bar{A}^{2}, g^{2}\right)$ such that $\left(t^{1}, \bar{A}^{1}, g^{1}\right) \leq^{3}\left(t^{2}, \bar{A}^{2}, g^{2}\right)$.

(4) If $\left(t^{1}, \bar{A}^{1}, g^{1}\right) \leq^{3}\left(t^{2}, \bar{A}^{2}, g^{2}\right)$, both in $\mathcal{T} \mathcal{T}$, and $\left(t^{1}, \bar{A}^{1}, g^{1}\right) \in \mathcal{T}_{\alpha}$ then $\left(t^{2}, \bar{A}^{2}, g^{2}\right) \in \mathcal{T} \mathcal{T}_{\alpha}$. 
Definition 3.17. (1) For $t \in \mathcal{T}$, let

$\operatorname{fsub}(t)=\left\{s \in \mathcal{T}:\right.$ for some finite $w \subseteq \operatorname{Suc}_{t}(\operatorname{rt}(t))$ we have

$$
s=\{\eta \in t: \neg(\exists \nu \in w)(\nu \unlhd \eta)\}\} .
$$

(2) For $\left(t^{1}, \bar{A}^{1}, g^{1}\right),\left(t^{2}, \bar{A}^{2}, g^{2}\right) \in \mathcal{T} \mathcal{T}$ we define the set $\mathcal{F} \mathcal{T}_{*}^{l}\left(\left(t^{1}, \bar{A}^{1}, g^{1}\right)\right.$, $\left.\left(t^{2}, \bar{A}^{2}, g^{2}\right)\right)$ to consist of all $f$ such that for some $t^{3} \in \operatorname{fsub}\left(t^{2}\right)$ we have

$$
f \in \mathcal{F T}^{l}\left(\left(t^{1}, \bar{A}^{1}, g^{1}\right),\left(t^{3}, \bar{A}^{2}\left\lceil t^{3}, g^{2}\left\lceil t^{3}\right)\right) .\right.\right.
$$

(3) $\left(t^{1}, \bar{A}^{1}, g^{1}\right) \leq_{*}^{l}\left(t^{2}, \bar{A}^{2}, g^{2}\right)$ if and only if $\mathcal{F} \mathcal{T}_{*}^{l}\left(\left(t^{1}, \bar{A}^{1}, g^{1}\right),\left(t^{2}, \bar{A}^{2}, g^{2}\right)\right)$ is not empty.

(4) Let

$\operatorname{ssub}(t)=\{s \in \mathcal{T}: s \subseteq t, \operatorname{rt}(s)=\operatorname{rt}(t)$ and for every $\eta \in s$,

$$
\left.\operatorname{Suc}_{t}(\eta) \backslash \operatorname{Suc}_{s}(\eta) \text { is finite }\right\} \text {. }
$$

Let $\mathcal{F} \mathcal{T}_{\otimes}^{l}$ be defined like $\mathcal{F} \mathcal{T}^{l}$ using $\operatorname{ssub}(t)$, and $\leq_{\otimes}^{l}$ be defined like $\leq_{*}^{l}$ but using $\mathcal{F} \mathcal{T}_{\otimes}^{l}$. $<_{*}^{l}$.

FACT 3.18. (1) $<_{*}^{l}$ is a partial order on $\mathcal{T} \mathcal{T}$ such that $<^{l}$ is a subset of

(2) Any $\leq_{*}^{l}$-increasing chain of length $\omega$ in $\mathcal{T}_{\mathcal{T}_{<\alpha}}$ has an upper bound in $\mathcal{T} \mathcal{T}_{\leq \alpha}$

(3) $\mathcal{T} \mathcal{T}_{\leq \omega_{1}}=\mathcal{T} \mathcal{T}_{<\omega_{1}}$

Proposition 3.19. (1) If $(t, \bar{A}, g) \in \mathcal{T} \mathcal{T}_{\leq \alpha}$, and $B \subseteq \omega$, then for some $\left(t^{\prime}, \bar{A}^{\prime}, g^{\prime}\right) \in \mathcal{T} \mathcal{T}_{\leq \alpha}$ we have:

$(\alpha)(t, \bar{A}, g) \leq^{3}\left(t^{\prime}, \bar{A}^{\prime}, g^{\prime}\right)$, in fact $t^{\prime} \in \operatorname{sub}^{-}(t)$,

( $\beta) \bigcup\left\{A_{\eta}^{\prime}: \eta \in t\right\}$ is a subset of $B$ or is disjoint from $B$.

(2) Similarly for $\mathcal{T} \mathcal{T}$.

Proof. (1) By induction on $\alpha$.

(2) Similarly. (Let for $\eta \in t, \mathcal{S}_{\eta}=\left\{s \in \mathcal{T}_{<\omega_{1}}: s \subseteq t, \operatorname{rt}(s)=\eta\right\}$ and let

$Z_{B}=\left\{\eta \in t:\right.$ for every $s_{1} \in \mathcal{S}_{\eta}$ there are $s_{2}, s_{1} \subseteq s_{2} \in \mathcal{S}_{\eta}$ and $\left(t^{\prime}, \bar{A}^{\prime}, g^{\prime}\right)$

such that $\left(s_{2}, \bar{A}\left\lceil s_{2}, g\left\lceil s_{2}\right) \leq^{3}\left(t^{\prime}, \bar{A}^{\prime}, g^{\prime}\right)\right.\right.$

and $\bigcup\left\{A_{r}: r \in s_{2}\right.$ is a subset of $\left.B\right\}$.

If $\operatorname{rt}(t) \in Z_{B} \cup Z_{\omega \backslash B}$ we are done; if not then we can find $\left(t^{\prime}, \bar{A}^{\prime}, g^{\prime}\right)$ satisfying $(\alpha)$ and such that $t \cap\left(Z_{B} \cup Z_{\omega \backslash B}\right)=\emptyset$, choosing $t^{\prime} \cap{ }^{n} \omega$ by induction on $n$; an easy contradiction.)

Proposition 3.20. If $(t, \bar{A}, g) \in \mathcal{T}_{\leq \alpha}$ and $E$ is an equivalence relation on $\omega$, then for some $\left(t, \bar{A}^{\prime}, g^{\prime}\right)$ and front $y$ of $t^{\prime}$ we have:

( $\alpha)(t, \bar{A}, g) \leq^{3}\left(t^{\prime}, \bar{A}^{\prime}, g^{\prime}\right)$,

( $\beta)$ for $\eta \in y, A_{\eta}^{\prime}$ is included in one E-equivalence class, 
$(\gamma)$ for $\eta \neq \nu$ from $y$, the E-equivalence classes in which $A_{\eta}^{\prime}, A_{\nu}^{\prime}$ are included, are distinct (hence disjoint),

$(\delta)$ on $A_{\mathrm{rt}\left(t^{\prime}\right)}^{\prime}$ we have: $E$ is either trivial or refines $\left\langle A_{\eta}^{\prime}: \eta \in \operatorname{Suc}_{t^{\prime}}\left(\operatorname{rt}\left(t^{\prime}\right)\right)\right\rangle$,

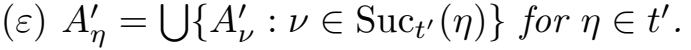

Proof. By induction on $\alpha$. (In clause $(\delta)$, the first possibility holds if the $y$ from $(\gamma)$ is $\{\operatorname{rt}(t)\}$, otherwise the second possibility holds.)

Proposition 3.21. (CH) Let I be an ideal such that $|\operatorname{Dom}(I)| \leq \aleph_{1}$ and

$$
\left(\forall X \in I^{+}\right)\left(\exists Y \in I^{+}\right)\left(Y \subseteq X \&|Y|=\aleph_{0}\right) .
$$

There is a $\leq_{*}^{0}$-increasing sequence $\left(\left\langle t^{\zeta}, \bar{A}^{1}, g^{\zeta}\right\rangle: \zeta<\omega_{1}\right)$ of standard members of $\mathcal{T}_{\mathcal{T}_{\leq \omega_{1}}}$ such that $\left\{\bigcup_{\eta \in t^{\zeta}} A_{\eta}^{1}: \zeta<\omega_{1}\right\}$ generates a non-principal ultrafilter $D$ on $\omega$ which is a $Q$-point, and for every equivalence relation $E$ on $\omega$ with no E-equivalence class belonging to $D$, for some $\zeta, E\left\lceil A_{\mathrm{rt}(t \zeta)}^{\zeta}\right.$ refines $\left\langle A_{\eta}^{\zeta}: \eta \in \operatorname{Suc}_{t}\left(\operatorname{rt}\left(t^{\zeta}\right)\right)\right\rangle$.

Proof. Straightforward; for limits use $3.18(2)+3.16(3)+3.16(2)$, for successors use $3.20+3.16(3)+3.16(1)$, (2).

Proposition 3.22. In 3.21, if in addition an ideal $I^{\prime}$ satisfies

(*) $\left|\operatorname{Dom}\left(I^{\prime}\right)\right| \leq \aleph_{1}$ and if $(t, \bar{A}, g) \in \mathcal{T} \mathcal{T}_{<\omega_{1}}$ and $g^{\prime}: \omega \rightarrow \operatorname{Dom}\left(I^{\prime}\right)$ then for some $\left(t^{\prime}, \bar{A}^{\prime}, g^{\prime}\right) \geq(t, \bar{A}, g)$ we have $g^{\prime}\left[A_{\langle\rangle}^{\prime}\right] \in I^{\prime}$,

then we can demand that $D$ is an $I^{\prime}$-ultrafilter (see 3.11(4)).

There are many problems on the $\sigma$-versions of cardinal invariants, and I think for some the method of [105], [130] is relevant, e.g.

Question 3.23 (see Brendle and Shelah [18]). Does $\chi_{\sigma}(D)=\chi(D)$ for all ultrafilters $D$ on $\omega$ ? Recall that

$$
\begin{aligned}
\chi(D)=\min \{|\mathcal{A}|: \mathcal{A} \subseteq D \text { and for every } A \in D, & \\
& \text { for some } B \in \mathcal{A} \text { we have } B \subseteq A\} \\
\chi_{\sigma}(D)=\min \{|\mathcal{A}|: & \mathcal{A} \subseteq{ }^{\omega} D \text { is such that for every } \bar{A} \in{ }^{\omega} D, \text { for some } \\
& \left.\bar{B} \in \mathcal{A} \text { we have }(\forall n<\omega)(\exists m<\omega)\left(B_{m} \subseteq \subseteq^{*} A_{n}\right)\right\} .
\end{aligned}
$$

So a reasonable scenario to prove the consistency of a negative answer is as follows: let, e.g., $\mu=\aleph_{\omega}$. We use FS iteration of c.c.c. forcing notions, $\left\langle\mathbb{P}_{i}, \mathbb{Q}_{j}: i \leq \delta^{*}, j<\delta^{*}\right\rangle$. We have $\mathbb{P}_{i}$-names $\underset{\sim}{D_{u}} i, A_{u, \gamma}\left(\right.$ for $\gamma<\gamma_{u}^{i}$ and $u \in[\mu]^{<\aleph_{0}}$ ) such that:

- ${\underset{\sim}{D}}_{u}^{i}$ is the filter on $\omega$ generated by $\left\{\underset{\sim}{A} A_{u, \gamma}: \gamma<\gamma_{u}^{i}\right\}$ and the co-bounded sets, 
- $\gamma_{u}^{i}$ are increasing with $i, D_{u}^{i} \subseteq D_{\sim}^{i}$ if $u \subseteq v$, and $D_{\sim}^{j} \subseteq D_{\sim}^{i}$ for $j<i$.

To simplify we decide:

(*) $\quad$ if $j<i, \Vdash_{\mathbb{P}_{j}}$ "A $\underset{\sim}{A} \omega$ and $u \in[\mu]<\aleph_{0}$ and $\underset{\sim}{A} \subseteq^{*}{ }_{\sim}^{A} A_{u, \gamma}$ for every $\gamma<\gamma_{u}^{j}$ " then $\mathbb{P}_{\mathbb{P}_{i}}$ "for every $v \in[\mu]^{<\aleph_{0}}, \underset{\sim}{A} \notin \underset{\sim}{D_{v}^{i}}$ ".

Also for the following it seems reasonable to try to be influenced by [105], [130].

Question 3.24 (see Brendle and Shelah [18]). Can $\pi \chi(D)$ be singular, where

$$
\begin{array}{r}
\pi \chi(D)=\min \left\{|\mathcal{A}|: \mathcal{A} \subseteq[\omega]^{\aleph_{0}}, \text { and for every } B \in D,\right. \\
\text { for some } \left.A \in \mathcal{A} \text { we have } A \subseteq^{*} B\right\} ?
\end{array}
$$

4. Nicely defined forcing notions. Rosłanowski and Shelah [92], and [115], [176] relate as algebraic three-dimensional varieties relate to manifolds in $\mathbb{R}^{n}$ and these, in turn, relate to general topology. In [115] (on nep and snep) and in Judah and Shelah [59] (on Suslin forcings) we deal with forcing notions defined in an absolute enough way; in [92] (more in [88], [89]) with forcing notions defined in an explicit way (say as trees and generally by creatures), and in [176] we deal with forcing notions related to the continuum.

Our problem with speaking about [92], [90], [88] and [89] is that much work is in progress; still orthogonal to it is the question whether in the main theorems of [92], all the assumptions are needed. That is, within the framework of conditions in the forcing being trees or $\omega$-sequences of creatures, are the demands on the norms necessary? This is dealt with for the conditions for properness in [92], showing necessity, but there are still gaps remaining.

QUestion 4.1. Are the sufficient conditions for properness in $[92, \S 2]$ necessary? The test case (chosen in [92]) is

$$
\mathbb{Q}=\left\{\left\langle w_{n}: n<\omega\right\rangle: w_{n} \subseteq 2^{n}, w_{n} \neq \emptyset \text { and } \lim _{n \rightarrow \omega}\left|w_{n}\right|=\infty\right\}
$$

ordered by $\bar{w} \leq \bar{w}^{\prime} \Leftrightarrow(\forall n \in \omega)\left(w_{n}^{\prime} \subseteq w_{n}\right)$.

Though properness is the main thing and there we look for counterexamples only for properness, it is interesting to know:

Question 4.2. Concerning other theorems of [92], are they sharp?

There are more specialized problems, probably solvable in this context.

QUESTION 4.3. Is there an ${ }^{\omega} \omega$-bounding forcing notion adding a perfect set of random reals?

It seems this should not be hard if true. 
The following problems (raised by Komjáth and Steprāns respectively) seem to me a matter of choosing the right variant of [92] or [88] and having the right finite combinatorics, but have failed.

Question 4.4. (1) Can each $A \in\left[{ }^{\omega} 2\right]^{\aleph_{1}}$ be null while the union of some $\aleph_{1}$ lines in $\mathbb{R} \times \mathbb{R}$ is not null?

(2) For reals $0<a_{0}<a_{1} \leq 1$, is it consistent with ZFC that: for $l<2$, $l=0$ iff some $A \in[\mathbb{R}]^{\aleph_{1}}$ has positive Hausdorff capacity for $a_{l}$ ?

I suppose that the feeling that the Cohen forcing notion and the random real forcing notion occupy a special place is old; probably more in the version speaking on the ideal of null sets and the ideal of meagre sets. I feel the former version is more interesting. For me this translates to

Problem 4.5. Among Suslin c.c.c. forcing notions, are Cohen forcing and random forcing special?

Some progress was made in [168].

THEOREM 4.6. If a Suslin c.c.c. forcing notion $\mathbb{Q}$ adds $\eta \in{ }^{\omega} \omega$ not dominated by any old $\nu \in{ }^{\omega} \omega$, then forcing with $\mathbb{Q}$ adds a Cohen real.

(The "Suslin" is needed for enough absoluteness, so with the existence of large cardinals we can allow a larger family.)

So the Cohen forcing notion is the minimal one among Suslin c.c.c. forcing notions adding an undominated real, so it is natural to conjecture:

Problem 4.7. Show that any Suslin c.c.c. forcing notion adding a real adds a Cohen real or adds a random real.

This really will show that Cohen and random are special.

In a sense the realm of Suslin c.c.c. forcing notions can be looked as being divided between the ${ }^{\omega} \omega$-bounding (with Random forcing as prototype) and those forcing notions adding an undominated real (with Cohen forcing as prototype); we can further distinguish those adding a dominating real.

However, the situation is very unbalanced: among Suslin c.c.c. forcing notions adding an undominated real we have many examples and a $<^{*}$ minimal one, Cohen (see Definition 4.8 below).

On the other hand we have no idea what occurs among the ${ }^{\omega} \omega$-bounding ones: probably random real is the unique one, but it is not out of the question that there is a plethora (adding one or many randoms is an irrelevant distinction; we can even order $(\mathbb{Q}, \underset{\sim}{r}), \underset{\sim}{r}$ a $\mathbb{Q}$-name of a real such that the order depends only on the subforcing $\underset{\sim}{r}$ generates). 
Definition 4.8. Let $\mathbb{Q}_{1}, \mathbb{Q}_{2}$ be definitions of forcing notions (absolute enough) say as in [115], or Suslin.

(1) $\mathbb{Q}_{1} \leq_{0}^{*} \mathbb{Q}_{2}$ if forcing with $\mathbb{Q}_{2}$ adds a generic for $\mathbb{Q}_{1}$ and we let $<^{*}$ mean $<_{0}^{*}$.

(2) $\mathbb{Q}_{1} \leq_{1}^{*} \mathbb{Q}_{2}$ means: for some $n$, if we force by iteration $n$ times of $\mathbb{Q}_{2}$, we add a generic for $\mathbb{Q}_{1}$.

(3) $\mathbb{Q}_{1} \leq_{2, \text { fs }}^{*} \mathbb{Q}_{2}$ is defined similarly using FS iteration of length $<\omega_{1}$.

(4) $\mathbb{Q}_{1} \leq_{2, \mathrm{cs}}^{*} \mathbb{Q}_{2}$ is defined similarly using CS of length $<\omega_{1}$.

Note: 4.7 is on the interval between the control measure problem (see Fremlin [37]) and von Neumann's question whether any complete c.c.c. Boolean algebra which as a forcing is ${ }^{\omega} \omega$-bounding, is a measure algebra. Another way to express the thought that Cohen and random are special was Kunen's conjecture; see Kunen [74], Kechris and Solecki [68], Solecki [189], [188] and Rosłanowski and Shelah [90].

It is natural to investigate the partial orders from 4.8. So,

Problem 4.9. (1) Investigate the quasi-order $\leq^{*}$ (and its variants) for $\mathbb{Q}$ which are nep (see $[115])$ or which are c.c.c. $\aleph_{0}$-nep or which arec.c.c. $\aleph_{0^{-}}$ snep. [We may concentrate on those with a generic real (those are the main interest for 4.13(1), (2) below).]

(2) Find a classification of such forcings, i.e.. find natural dividing lines (properties which, together with their negations, have serious consequences, so you may prove by division to cases).

An example is (and probably not hard):

Question 4.10. Prove that dominating real forcing (i.e., the Hechler forcing notion) is $\leq{ }^{*}$-minimal among Suslin c.c.c. forcing notions adding a dominating real. [For $\leq_{1}^{*}$ this is easy.]

Looking more serious are

Question 4.11. Can you characterize the $<^{*}$-minimal $\mathbb{Q}$ which add a Cohen real but are not equivalent to the Cohen forcing (hopefully there is one or at least there are only few)?

QUEsTion 4.12. Can you characterize the $<{ }^{*}$-minimal $\mathbb{Q}$ among the nonminimal $\mathbb{Q}$ which add a dominating real but are stronger than the Hechler forcing notion?

A positive solution of 4.7 would also show that the only symmetric Suslin c.c.c. forcing notions are the Cohen forcing and the random forcing (by $[115, \S 9])$. 
Why should we be interested in Suslin proper or in nep forcing or better yet, why am I? The reason has been iteration theorems; when you are interested in iterating some very special forcing notions, the proof of their properness gives more, e.g., the existence of generic conditions over models occurs also for countable models of versions of ZFC which are not necessarily $\prec(\mathcal{H}(\chi), \in)$. Moreover, some things are preserved by iterations and this is helpful for specific problems, which is the point of Judah and Shelah [59]. [In [59] this was phrased using descriptive set theory, getting Suslin proper. However, this does not cover the Sacks forcing notion, the Laver forcing notion, etc., which was accomplished by nep.]

Needless to say, I think iteration theorems for forcing are important and interesting (otherwise, normally I would not have written a book on the subject-see $\S 2$ ).

Another basic reason is that the family of nep forcing notions forms a natural class. Now, while I feel that general sets are much more basic and interesting then families of definable ones, and so prefer $\mathcal{P}(\mathcal{P}(\omega))$ to the family of projective sets, certainly they are interesting and natural.

Another reason is "large" ideals. Let $I$ be a $\kappa$-complete ideal on $\lambda$. Gitik and Shelah [44] start by proving that $\mathcal{P}(\kappa) / I$ cannot be (the Boolean algebra which up to isomorphism is equivalent to) the Cohen forcing or random real forcing, an old question which Fremlin promoted (see [38]), which comes from asking: can the classical result of Solovay [190] (saying that consistently $2^{\aleph_{0}}$ is real valued measurable, now the Maharam type there was large) be improved to get small Maharam type.

But then [44] turns to:

Problem 4.13. (1) Prove that $\mathcal{P}(\kappa) / I$ cannot be a Suslin c.c.c. forcing generated by the name of one real $\eta$ (where $I$ is a $\kappa$-complete ideal on $\kappa$ or at least $\aleph_{1}$-complete).

(2) Similarly for Suslin proper (or weaken the definability demandnatural as the existence of the ideal implies more absoluteness).

(3) Even reasonable subclasses or cases are interesting.

Problem 4.14. Similarly, we can ask about a $\sigma^{+}$-complete ideal $I$ on $\kappa$ such that $\mathcal{P}(\kappa) / I$ has a dense subset isomorphic to a partial order defined in $\left(\mathcal{H}_{<\sigma}\left(\kappa^{\prime}\right), \in\right)$ with parameters.

In Gitik and Shelah [44], [46], [42], in addition to information on adding not too many random or Cohen reals, and (toward 4.13) to general criteria for impossibility, we consider more specific cases (see then [179]). The problems have led us to properties of definable forcing notions like symmetry. The theorems on Cohen and random reals use the symmetry (i.e. the Fubini theorem), but other properties pop up naturally, e.g., for Suslin c.c.c. forcing $\mathbb{Q}$ with a dominating real $\eta$ as generic, to show impossibility it suffices 
to show: $\vdash_{\mathbb{Q}}$ "b $=\aleph_{1}$ " (by [44]). Maybe the work on the ideals is done and we just need to verify that always at least one of the criteria applies (at least for large subclasses). Now [168], [115, §8, §9] come to my mind.

Considerations like this lead to questions like

Question 4.15. Find sufficient conditions on $\mathbb{Q}$ for " $\mathbb{Q} *$ Random/Random adds no random real".

(This question is chosen since it is also interesting because if the condition is reasonable enough, it suffices for proving $\mathrm{CON}\left(\operatorname{cov}^{*}\right.$ (null $)<$ non(meagre)), see Bartoszyński, Rosłanowski and Shelah [9], [8].)

QUESTION 4.16. Investigate commuting pairs (see [115]).

For such considerations I felt that a peculiar property of Cohen forcing and random forcing is their satisfying: "being a maximal antichain is a Borel property"; this leads to

Definition 4.17. A forcing notion $\mathbb{Q}$ is very Suslin c.c.c. if it is Suslin c.c.c. and also the notion of " $\left\{r_{n}: n<\omega\right\}$ is a maximal antichain" is $\Sigma_{1}^{1}$.

We hope this will turn out to be a good dividing line of the Suslin c.c.c. forcing (so helping to prove theorems). This is because I suspect the answer to the following is yes.

Question 4.18. Prove: If $\mathbb{Q}$ is a Suslin c.c.c. forcing notion, say with generic real $\underset{\sim}{r}$, and it is not very Suslin c.c.c. above any $p \in \mathbb{Q}$ then $\Vdash_{\mathbb{Q}}$ "b $=\aleph_{1} "$. (This should help 4.13 by $[44, \S 4]$.) See on this [116].

As in [115] we can define (restricting $\kappa$ to be $\aleph_{0}$ for simplicity)

Definition 4.19. (1) A forcing notion $\mathbb{Q}$ is $\omega-n w-n e p$ if there is a sequence $\bar{\varphi}=\left\langle\varphi_{0}, \varphi_{1}, \varphi_{2}\right\rangle$ of $\Sigma_{1}^{1}$ definitions such that:

(a) the set of members of $\mathbb{Q}$ and $\leq \mathbb{Q}$ are $\Sigma_{1}^{1}$ sets (of reals) defined by $\varphi_{0}, \varphi_{1}$, respectively,

(b) if $N$ is an $(\bar{\varphi}, \omega)$-nw-candidate (that is, a model of $\mathrm{ZFC}_{*}^{-}$, suitable version of ZFC, not necessarily well-founded but with standard $\omega)$, and with the real parameters of the $\varphi_{l}$ 's, and $p \in \mathbb{Q}^{N}=\{x$ : $\left.N \models \varphi_{0}(x)\right\}$, then for some $q \in \mathbb{Q}$, we have

( $\alpha) p \leq q$,

$(\beta) q$ is $(N, \mathbb{Q})$-generic, which means that for every $\mathcal{I} \in \operatorname{pd}(N, \mathbb{Q})$ $=\{\mathcal{J} \in N: N \models$ $\mathcal{J}$ is predense in $\mathbb{Q} "\}$, some list $\left\langle r_{n}:\right.$ $n<\omega\rangle$ of $\{x: N \mid=x \in \mathcal{I} "\}$ is predense above $q$,

$(\gamma) \varphi_{2}\left(q,\left\langle r_{n}: n<\omega\right\rangle\right)$ holds,

(c) if $\varphi_{2}\left(q^{\prime},\left\langle r_{n}^{\prime}: n<\omega\right\rangle\right)$ then $\left\{r_{n}^{\prime}: n<\omega\right\}$ is predense above $q^{\prime}$.

(2) Omitting the "nw" or writing just "w" means we allow only wellfounded candidates. 
(3) [On Ur see below.] We say $\underset{\sim}{r} \subseteq \mathbf{U r}$ ( or $\underset{\sim}{r} \subseteq \mathcal{H}_{<\aleph_{1}}$ (Ur) in the w-case) is generic for $\mathbb{Q}$ if: $\underset{\sim}{r}$ is a $\mathbb{Q}$-name and " $a \in \underset{\sim}{r}$ " is determined by the truth value $\varphi_{\sim}^{r, a}\left[G_{\mathbb{Q}}\right]$ and the sequence $\left\langle\varphi_{\underset{\sim}{r}, a}: a\right\rangle$ is definable in $\mathfrak{B}_{\mathbb{Q}}$ (see [116]).

Now, there are more examples of $\omega$-nw-nep forcing notions in addition to Cohen and random: say the Sacks forcing notion. But of the Laver forcing notion we should beware; note that we can guarantee that for every $\mathcal{I} \in \operatorname{pd}(\mathbb{Q}, N)$, for some front $X$ of $q$ we have $\eta \in X \Rightarrow q^{[\eta]}$ is above a member of $\mathcal{I}$, but being a front is not absolute from $(\bar{\varphi}, \omega)$-nw-candidates, as they are not necessarily well-founded. In fact, we can easily craft a counterexample: assume $N \models$ " $\alpha$ a countable ordinal", but from the outside not well-ordered. There are $f, T \in N$ (so actually $f=f_{\alpha}, t=T_{\alpha}$ ) such that

$N \models " T \subseteq{ }^{\omega>} \omega$ is closed under initial segments, \langle\rangle$\in T$,

$$
\begin{aligned}
& f: T \rightarrow \alpha+1, \quad f(\langle\rangle)=\alpha, \\
& f(\eta)>0 \Rightarrow(\forall n)(\eta \frown\langle n\rangle \in T), \\
& f(\eta)=0 \Rightarrow(\forall n)(\eta \frown\langle n\rangle \notin T), \\
& f(\eta)=\beta+1 \Rightarrow(\forall n<\omega)[\eta \frown\langle n\rangle \in T \& f(\eta \frown\langle n\rangle)=\beta], \\
& \text { if } f(\eta)=\delta \text { is limit } \\
& \text { then }\langle f(\eta \frown\langle n\rangle): n\langle\omega\rangle \text { is strictly increasing with limit } \delta " .
\end{aligned}
$$

Let

$$
\begin{aligned}
\mathcal{I}_{0} & =\left\{(\omega>\omega)^{[\eta]}: \eta \in T, f(\eta)=0\right\}, \\
\mathcal{I}_{n+1} & =\left\{(\omega>\omega)^{[\eta-\varrho]}: \eta \in \mathcal{I}_{n}, \varrho \in T, f(\varrho)=0\right\} .
\end{aligned}
$$

Clearly, above no $q$ is every $\mathcal{I}_{n}$ predense.

Still, we have

THEOREM 4.20. The ${ }^{\omega} \omega$-bounding and almost ${ }^{\omega} \omega$-bounding forcing notions covered by [92] and [88] are all $\omega-n w-n e p$.

What about iterations? Now, to unify the treatment of finite support and countable support we revise our definition to have two quasi-orders $\leq^{\mathbb{Q}}, \leq_{\mathrm{pr}}^{\mathbb{Q}}$ such that $p \leq_{\mathrm{pr}} q \Rightarrow p \leq q$. Hence in Definition 4.19 we add $\varphi_{1,2}$ (absolute just like $\varphi_{1}$ ) serving as a definition of $p \leq_{\mathrm{pr}} q$. The support is countable but finite for the apure cases, i.e., $\neg\left(\emptyset_{\alpha} \leq\right.$ pr $\left.p(\alpha)\right)$ only for finitely many $\alpha$. First assume the length is $\alpha^{*}<\omega_{1}$, so we can use a parameter coding a well-ordering on $\omega$ with this order type. We should repeat the proof in [115] in order to prove preservation in this case, but we'd better not use the $L_{\omega_{1}, \omega}$-completeness as there, as we have problem with well-foundedness. So we just demand: elements in $\mathbb{P}_{\alpha}$ have depth $<\omega \alpha$ (or so).

What about long iterations? It seems to me, at least now, better (and fit to [115], too) to use a set of urelements $\mathbf{U r}$; let $\mathfrak{C}$ [and $\mathfrak{B}$ ] be models with universes $\mathbf{U r}[$ or $\subseteq \mathbf{U r}]$ and $\mathcal{S} \subseteq[\mathbf{U r}] \leq \aleph_{0}$ be unbounded (usually 
stationary, if $a \in \mathcal{S}$ then $\mathfrak{B} \mid a \subseteq \mathfrak{B}$ and $\mathfrak{C}\lceil a \subseteq \mathfrak{C}$ ), and anyhow the family of nw-candidates should be $\left(<\aleph_{1}\right)$-directed and uord $\subseteq \mathbf{U r}$ is a well-ordered set (which will serve as the length of the iteration). Now, a candidate will be a countable model $N \subseteq(\mathcal{H}(\chi), \in)$ of $\mathrm{ZFC}_{*}^{-}$(where $\mathcal{H}(\chi)$ includes the urelements), $N \cap \mathbf{U r} \in S$, where $\mathbf{U r}$ and the relations of $\mathfrak{B}$ and $\mathfrak{C}$ are the considered relations. We define an nw-candidate similarly but now $\in^{N}$ is a relation on $N$ and $N$ is not necessarily well-founded (but the order type of the well-ordered ordinals of $N$ is $>\operatorname{otp}(N \cap$ uord). In Definition 4.19 we demand, of course, that $\varphi_{l}$ are upward absolute from the nw-candidates. Now we can use uord as the index set for iteration (instead of the true ordinals) and there are no problems.

This set-up looks like a nice army with no enemy yet, but this seems to me a natural dividing line among the nep forcing notions and therefore reasonable for our interest in those forcing notions per se. I hope it will help, particularly with 4.13 (and even more so by the c.c.c. version, see 4.22 below).

More on preservation (for not necessarily c.c.c. forcing notions), commutativity, associativity of generic sets, and countable for pure/finite for apure support iterations see [116].

A restricted version of the large continuum is

Problem 4.21. Can we have long $\left(>\omega_{2}\right)$ iterations of ${ }^{\omega} \omega$-bounding forcing notions (or at least nw-nep ones) not collapsing cardinals and not adding Cohen reals?

What about the c.c.c. (nw-nep) ones (or even very Suslin c.c.c.) ones?

Definition 4.22. $\mathbb{Q}$ is c.c.c.-nw-nep if it is a pair $\left\langle\varphi_{0}, \varphi_{1}\right\rangle$ of formulas such that:

$(\alpha) \mathbb{Q}=\bigcup\left\{\mathbb{Q}^{N}: N\right.$ is an nw-candidate $\} \subseteq{ }^{\omega}$ uord, similarly $\leq_{Q}=\bigcup\left\{\leq_{\mathbb{Q}}^{N}:\right.$ $N$ is an nw-candidate $\}$; recall that in $N, \mathbb{Q}^{N}, \leq_{\mathbb{Q}}^{N}$ are defined by the appropriate $\varphi_{l}$,

$(\beta)$ all $\varphi_{l}$ are upward absolute among nw-candidates,

$(\gamma)$ if $N \models$ " $\mathcal{I} \subseteq \mathbb{Q}$ is predense", then $\mathcal{I}^{\mathbb{Q}}$ is really predense.

Proposition 4.23. (1) The Cohen and random forcing notions are c.c.c.-nw-nep.

(2) The class of c.c.c.-nw-nep is closed under FS iterations.

(3) This class is also closed under subforcings.

And I am curious to know:

Problem 4.24. Does 4.23 exhaust all c.c.c-nw-nep forcing notions (at least those with a generic real)? Can we give a full characterization of such forcing notions? 
Being interested in classifying nep c.c.c. forcing notions, we may consider sweetness; the discussion below is in fact an introduction to [89]. Sweetness phenomenons are when we can build homogeneous forcing notions (as in $[146, \S 7, \S 8]$ ); sour phenomenons are strong negations (as in $[146, \S 6]$ ).

Problem 4.25. For which $(\mathbb{Q}, \eta)$, nep c.c.c. forcing notions, is it consistent that:

(a) there is a $(\mathbb{Q}, \eta)$-generic real over $\mathbf{L}[A]$ for every $A \subseteq \omega_{1}$, and

(b) for every subset $B \in \mathbf{L}[\mathbb{R}]$ of ${ }^{\omega} 2$, for some $A \subseteq \omega$, for a dense set of $p \in\left(\mathbb{Q}, \eta_{\sim}\right)$ : for some truth value $\mathbf{t}$, if $\eta$ is a $\left(\mathbb{Q}_{\geq p}^{\mathbf{L}[A]}, \underset{\sim}{\eta}\right)$-generic real then $\eta \in B \Leftrightarrow \mathbf{t}$ ?

So for this question, random reals are complicated (see $[146, \S 6])$, whereas Cohen real and universal-meagre one (and dominating $=$ Hechler reals) are low, see Judah and Shelah [62].

More generally,

Problem 4.26. Let $(\mathbb{Q}, \eta)$ be a nep c.c.c. forcing notion, and $\kappa$ be a cardinal number. Let $I_{(\mathbb{Q}, \eta)}^{\kappa}$ be the $\kappa$-complete ideal generated by sets of the form

$$
A_{N}=\{\eta: \eta \text { is not }(\mathbb{Q}, \underset{\sim}{\eta}) \text {-generic over } N\}
$$

for countable models $N \prec(\mathcal{H}(\chi), \in)$ to which $(\mathbb{Q}, \eta)$ belongs. What is the consistency strength of "every projective set, or even every set from $\mathbf{L}[\mathbb{R}]$, is equal to a Borel set modulo $\left.I_{(\mathbb{Q}, \eta \sim}^{\kappa}\right)$ " ?

We hope for a strong dichotomy phenomenon, i.e., if the answer above is negative for $(\mathbb{Q}, \eta)$ (so the sweetness fails), then a strong negation holds, so we call such phenomena sourness.

Definition 4.27. Let $\mathbb{Q}_{1}, \mathbb{Q}_{2}$ be nep c.c.c. forcing notions definable in $\mathbf{L}$ (or $\mathbf{L}[r]$ ). We say that $\mathbb{Q}_{1}, \mathbb{Q}_{2}$ are explicitly sour over Cohen if we can find $\mathbb{Q}_{l}$-names $\eta_{l}$ of Cohen reals (for $\left.l=1,2\right)$ such that if $G_{l} \subseteq \mathbb{Q}_{l}$ is generic over $\mathbf{L}($ or $\mathbf{L}[r]) \tilde{)}$ for $l=1,2$ then $\eta_{1}\left[G_{1}\right] \neq \eta_{2}\left[G_{2}\right]$.

We should note that there may be homogeneity for wrong reasons, i.e., maybe when we force, very few $(\mathbb{Q}, \eta)$-generic reals over $\mathbf{L}$ are added and then homogeneity holds for "degenerate" reasons; we may call such cases saccharin. For more on this direction see [88].

Speaking about the class of sweet forcing notions we should mention the following problem. 
Problem 4.28. For any cardinal $\kappa$ and a large cardinal property (or consistency strength) we may ask the following.

(1) Is there a widest class $\mathcal{K}$ of absolute enough forcing notions such that for some forcing notion $\mathbb{P}$ we have

(a) $\Vdash_{\mathbb{P}} \kappa=\aleph_{1}$,

(b) $\mathbb{P}$ is homogeneous for complete subforcings from $\mathcal{K}$,

(c) if $\mathbb{P}^{*} \lessdot \mathbb{P}$ and $\mathbb{P}^{*}$ has a generic real then $\mathbb{P} / G_{\mathbb{P}^{*}}$ satisfies (b) in $\mathbf{V}\left[G_{\mathbb{P}^{*}}\right]$ ?

(2) If not, at least give a wide enough such class.

(3) Are there two classes $\mathcal{K}_{1}, \mathcal{K}_{2}$ as above such that there is no class with the respective property including $\mathcal{K}_{1} \cup \mathcal{K}_{2}$ ? Or even that the consistency strength of the (now) obvious conclusion is higher than the given one?

Now, the variants of sweetness try to deal with the case of $\kappa=\aleph_{1}$ and the consistency strength ZFC (see Rosłanowski and Shelah [89, §3]); the theory of determinacy is applicable to the case $\kappa=\aleph_{1}$ and maximal consistency strength (see Woodin [198]), and [125] intends to deal with the case of "ZFC $+\kappa$ is strongly inaccessible" (and no further consistency strength assumptions).

Question 4.29. Is there a sweet forcing notion (see [146, §7]), preferably a natural one, such that it cannot be completely embedded into the forcing notion constructed in [146, §7] (it was obtained by composing $\mathcal{U M}$, amalgamating and direct limits), or at least not "below $\mathcal{U} \mathcal{M}$ " (in the sense of $\leq_{1}^{*}$, see 4.8$)$ ?

Let me now mention free iterations:

In nep (also above) we can replace CS by free limit as in [142, Ch. IX]. (This was my third proof for preservation of properness (the first was like $[116, \S 2]$, the second was like the one in [142, Ch. III]; this third proof looks very natural but no real reason for replacing CS iteration by it has appeared.)

In particular

Proposition 4.30. The definition of $L_{\omega_{1}, \omega}$-free iteration is absolute enough, so we have our $\varphi_{2}$ (we ignore the nw).

Preservation (of reasonable properties) by CS iterations of proper forcing (or variants) seems to me a worthwhile subject. For ${ }^{\omega} \omega$-bounding the situation is nice, "proper $+{ }^{\omega} \omega$-bounding" is preserved, and analogous results hold for a large family of properties, even, e.g., " $D$ generates a $P$-point ultrafilter on $\omega$ ". But some properties do not fit, though still are preserved in 
limits (see [176, Ch. XVIII, §3]). For example, below we shall consider the case of " $A \subseteq{ }^{\omega} 2$ is non-null". The simple preservation is: in the existence of generic conditions we can preserve "a given old $\eta \in{ }^{\omega} 2$ is random over the model $N\left[G_{\mathbb{P}}\right] "$.

Question 4.31. Assume that:

(a) $\overline{\mathbb{Q}}$ is a CS iteration, or an $L_{\omega_{1}, \omega}$-free iteration,

(b) each $\mathbb{Q}_{\alpha}$ is proper, or a nep forcing notion,

(c) each $\mathbb{Q}_{\alpha}$ is "non-null for $(\mathbb{S}, \underset{\sim}{r})$-preserving" ( ${ }^{3}$ ) (if each $\mathbb{Q}_{\alpha}$ is nep and $\underset{\sim}{r}$ is, e.g., random, then by [115] this is equivalent to not making old ${ }^{\omega} 2$ null).

Does $\mathbb{P}_{\alpha}$ have the property from clause (c)? (so we have four versions of the question, as for clause (a) and for clause (b) we can choose the first or the second possibilities). Can we generalize to other natural ( $\aleph_{1}$-complete) ideals?

Some cases are known; first we discuss the free iteration case. Assume:

(*) each $\underset{\sim}{\mathbb{Q}}{ }_{i}$ has a generic ${\underset{\sim}{r}}_{i} \subseteq \underset{\sim}{\alpha}$.

(Hence the $\mathbb{P}_{\alpha}$ have this property as it is preserved.)

We assume knowledge of free iterations (see [176, IX, §1, §2]); in short, if $\mathbb{P}_{n} \lessdot \mathbb{P}_{n+1}$ for $n<\omega$, let $\mathbb{P}_{\omega}$ be

$\left\{\psi: \psi\right.$ is a sentence in the $L_{\omega_{1}, \omega}$ propositional calculus with

the set of propositional variables $\bigcup\left\{\mathbb{P}_{n}: n<\omega\right\}$ such that in some forcing extension of $\mathbf{V}$ there is $G \subseteq \bigcup\left\{\mathbb{P}_{n}: n<\omega\right\}$ satisfying

(a) for each $n<\omega, G \cap \mathbb{P}_{n}$ is generic over $\mathbf{V}$,

(b) looking at $G$ as assigning truth values to members of $\bigcup\left\{\mathbb{P}_{n}: n<\omega\right\}$, it assigns the value truth to $\left.\psi\right\}$.

The order of $\mathbb{P}_{\omega}$ is the natural one.

We now concentrate on the case of an $L_{\omega_{1}, \omega}$-free iteration $\overline{\mathbb{Q}}$ such that $\Vdash_{\mathbb{P}_{i}}$ "Q্Q $\mathbb{Q}_{i}$ is nep and $\left({ }^{\omega} 2\right) \mathbf{V}^{\mathbb{P}_{i}}$ is not null in $\left({ }^{\omega} 2\right) \mathbf{V}^{\mathbb{P}_{i} * \mathbb{Q}_{i}} "$

This is quite a wide case. What does it mean for a successor $\alpha=\lg (\overline{\mathbb{Q}})$ ? Say, $\alpha=2$ so we know that in $\mathbf{V}^{\mathbb{Q}_{0}},\left(\omega_{2}\right)^{\mathbf{V}}$ is not null, and in $\mathbf{V}^{\mathbb{Q}_{0} * \mathbb{Q}_{1}}$, $\left({ }^{\omega_{2}}\right)^{\mathbf{V}^{\mathbb{Q}_{0}}}$ is not null. But for nep forcing notions, preserving the non-nullity of ${ }^{\omega} 2$ implies preserving the non-nullity of any old non-null set by $[115, \S 7]$. Hence there is no problem with successor stages and the case $\operatorname{cf}(\alpha)>\aleph_{0}$ is trivial. So now assume $\delta=\lg (\overline{\mathbb{Q}})$ is a limit ordinal of countable cofinality.

$\left({ }^{3}\right) \mathbb{S}$ is a nep forcing notion, $r$ is a hereditarily countable $\mathbb{S}$-name, so $(\mathbb{S}, r)$ induces an ideal on ${ }^{\omega} 2 ; \mathbb{S}$ may be random and then the ideal is the ideal of null sets. 
As $\mathbb{P}_{\delta}$ is nep it is enough to assume

(**) $\quad p_{0} \Vdash_{P_{\delta}}$ "T $\underset{\sim}{ } \subseteq \omega>2$ is a subtree and $\operatorname{Leb}(\lim (\underset{\sim}{T}))>0$ ", and $\delta=$ $\bigcup_{n<\omega} i_{n}, i_{n}<i_{n+1}$, and without loss of generality $\underset{\sim}{T} \cap n \geq 2$ is a hereditarily countable $\mathbb{P}_{i_{n}}$-name,

and to find an old $\eta \in{ }^{\omega} 2$ such that $p_{0} \nVdash_{\mathbb{P}_{\delta}} " \eta \notin \lim (\underset{\sim}{T})$ ". Now, let $N$ be a $\overline{\mathbb{Q}}$ candidate to which $\left\{\underset{\sim}{T}, p_{0},\left\langle i_{n}: n<\omega\right\rangle\right\}$ belongs (without loss of generality $N \prec(\mathcal{H}(\chi), \in)$, with $\chi$ large enough). Let $G_{0} \subseteq \operatorname{Levy}\left(\aleph_{0},\left(2^{\left|\mathbb{P}_{\delta}\right|}\right)^{N}\right)$ be generic over $N$. Let $p_{1} \in N\left[G_{0}\right]$ be such that $p_{1} \in\left(\mathbb{P}_{\delta}\right)^{N\left[G_{0}\right]}, N\left[G_{0}\right] \models$ " $p_{0} \leq p_{1}$ and $p_{1}$ is explicitly $\left(N, \mathbb{P}_{\delta}\right)$-generic". Let $G_{1}=G_{1, \delta} \subseteq \mathbb{P}_{\delta}^{N\left[G_{0}\right]}$ be generic over $N\left[G_{0}\right]$ such that $p_{1} \in G_{1, \delta}$ and let $G_{1, i_{n}}=G_{1} \cap \mathbb{P}_{i_{n}}^{N\left[G_{0}\right]}$. Let $s$ be a random real over $N\left[G_{0}\right]\left[G_{1}\right]$ (if we replace random by other nep-explicitly demand even more models), hence over $N$ too. Clearly we can choose such $G_{0}, G_{1, \delta}, s$ in V. So $N[s]$ is a class (= definable) of $N\left[G_{0}\right]\left[G_{1}\right][s]$, and clearly $N[s]$ is a $\overline{\mathbb{Q}}$-candidate. Also, there is $s^{\prime} \in \lim \left(\underset{\sim}{T}\left[G_{1}\right]\right), s^{\prime} \equiv s$ (i.e., $s^{\prime} \in{ }^{\omega_{2}}$ and the set $\left\{l<\omega: s^{\prime}(l) \neq s(l)\right\}$ is finite). Let us define $\psi$ as

$$
\psi=p \& \bigwedge_{n<\omega}\left[s^{\prime} \mid n \in\left(\underset{\sim}{T} \cap{ }^{n} 2\right)\left[G_{\mathbb{P}_{i_{n}}}\right]\right]
$$

(note that, by the assumption, there is $\left\langle\psi_{n, \eta}: n<\omega, \eta \in{ }^{n} 2\right\rangle, \psi_{n, \eta}$ an $L_{\omega_{1}, \omega}$-sentence for $\mathbb{P}_{i_{n}}$, i.e. using (countably many) variables $q \in \mathbb{P}_{i_{n}}$, such that

$$
p_{0} \Vdash " \eta \in \underset{\sim}{T} \cap{ }^{n} 2 \text { iff } \psi_{n, \eta} \in G_{P_{i_{n}}} "
$$

for $n<\omega, \eta \in{ }^{n} 2$, so, up to equivalence,

$$
\psi=p \& \bigwedge_{n} \psi_{n, s^{\prime} \uparrow n}
$$

(recall that $\underset{\sim}{T} \cap{ }^{n} 2$ is a $\mathbb{P}_{i_{n}}$-name). The problem is whether $\psi \in \mathbb{P}_{\delta}$. Now, $\psi \in N[s]$, so by absoluteness (i.e. preservation of being nep) to show $\psi \in \mathbb{P}_{\delta}$ it suffices to show that this holds in $N[s]$.

So we need

$$
N[s] \models " \psi \& \bigwedge_{n} \Psi_{\mathbb{P}_{i_{n}}} \text { has a Boolean-valued model", }
$$

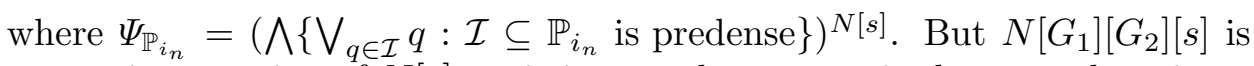
a generic extension of $N[s]$, so it is enough to prove it there; so there is no problem. So for free iteration of nep forcing for the case of "non-null" the answer is yes (for CS iterations use [176, Ch. XVIII, 3.8, pp. 912-916]).

On the other hand, in full generality the answer to 4.31 is no; note that life is harder if we want to preserve positiveness for $I_{(\mathbb{Q}, \eta)}$, where $\mathbb{Q}$ is (nep but) not c.c.c.; on this and more see [116]. 
Another possible direction is

Problem 4.32. (1) Is there an interesting theory of nicely definable forcing notions in $\mathcal{H}(\theta)$ or $\mathcal{H}_{<\theta}(\sigma)$ ?

(2) Similarly for the theory of iterations (see later).

(3) Generalize [92], replacing $\aleph_{0}$ by $\kappa$. Probably require $\kappa=\kappa^{<\kappa}>\aleph_{0}$ or even that $\kappa$ is strongly inaccessible.

E.g., let for simplicity $\kappa$ be strongly inaccessible, $D^{*}$ a normal filter on $\kappa$. For a cardinal $\theta<\kappa$ let a $\theta$-creature $\mathfrak{c}$ consist of $\left(R^{\mathfrak{c}}, \operatorname{pos}^{\mathfrak{c}}, \operatorname{val}^{\mathfrak{c}}\right)$, where: $R^{\mathfrak{c}}$ is a $\theta^{+}$-complete forcing notion, $\operatorname{pos}^{\mathfrak{c}}$ is a non-empty set, $\mathrm{val}^{\mathfrak{c}}$ is a function from $R^{\mathfrak{c}}$ to $\mathcal{P}\left(\operatorname{pos}^{\mathfrak{c}}\right) \backslash\{\emptyset\}$ such that $R^{\mathfrak{c}} \models " x \leq y " \Rightarrow \operatorname{val}^{\mathfrak{c}}(y) \subseteq \operatorname{val}^{\mathfrak{c}}(x)$.

A $\kappa$-normed tree is $\left(T^{*}, \overline{\mathfrak{c}}, \bar{\theta}\right)$, where $T^{*} \subseteq{ }^{\kappa>}$ Ord is a subtree, usually closed under increasing sequences of length $\left\langle\kappa, \overline{\mathfrak{c}}=\left\langle\mathfrak{c}_{\eta}: \eta \in T^{*}\right\rangle, \mathfrak{c}_{\eta}\right.$ is a $\theta_{\eta^{-}}$-creature, $\bar{\theta}=\left\langle\theta_{\eta}: \eta \in T^{*}\right\rangle$ and $\operatorname{pos}^{\mathfrak{c}_{\eta}}=\operatorname{Suc}_{T^{*}}(\eta)$.

We can consider

$$
\begin{aligned}
\mathbb{Q}_{\left(T^{*}, \overline{\mathfrak{c}}^{*}, \bar{\theta}\right), D^{*}}^{1}= & \mathbb{Q}^{1} \\
=\{(T, \bar{r}): & \text { (a) } T \text { is a subtree of } T^{*},(<\kappa) \text {-closed, } \\
& \text { (b) } \left.\bar{r}=\left\langle r_{\eta}: \eta \in \operatorname{sp}(T)\right)\right\rangle, r_{\eta} \in R^{\mathfrak{c}_{\eta}} \text { and } \operatorname{val}^{\mathfrak{c}_{\eta}}\left(r_{\eta}\right)=\operatorname{Suc}_{T}(\eta) \\
& \text { if } \eta \in \operatorname{sp}(t), \\
& \text { (c) for every } \eta \in \lim _{\kappa}(T), \text { we have } \\
& \left\{\zeta<\kappa: \eta\lceil\zeta \in \operatorname{sp}(T)\} \in D^{*}\right\}
\end{aligned}
$$

with the natural order, where $\operatorname{sp}(T)=\left\{\eta \in T:\left(\exists^{\geq 2} x\right)[\eta \frown\langle x\rangle \in T]\right\}$. Trivially, forcing with $\mathbb{Q}^{1}$ adds neither bounded subsets of $\kappa$ nor sequences of ordinals of length $<\kappa$. For this forcing notion, if $T_{\alpha}^{*}:=\left\{\eta \in T^{*}: \lg (\eta)=\alpha\right\}$ has cardinality $<\kappa$ for each $\alpha<\kappa$, and $\left(\forall \eta \in \lim _{\kappa}\left(T^{*}\right)\right)\left[\left\{\zeta<\kappa: \theta_{\eta \uparrow \zeta}>\zeta\right\}\right.$ $\left.\in D^{*}\right]$ (or there is $A \in D^{*}$ such that $\zeta \in A \& \eta \in T \& \lg (\eta)=\zeta \Rightarrow \theta_{\eta}>\zeta$ ), then forcing with $\mathbb{Q}_{\left(T^{*}, \overline{\mathrm{c}}^{*}, \bar{\theta}\right), D^{*}}^{1}$ does not collapse $\kappa^{+}$. Also, this forcing is $(<\kappa)$-complete ${ }^{\kappa} \kappa$-bounding and $\mathbb{Q}^{1}$-names of $\underset{\sim}{\tau}: \kappa \rightarrow$ Ord can be read continuously on $T$ for a dense set of $(T, \bar{r}) \in \mathbb{Q}^{1}$. Moreover, this property is preserved by $(\leq \kappa)$-support iterations (by $[110, \S 1]$, or directly).

We can allow gluing (i.e., putting together with $\eta$ many nodes above, creating a new forcing notion, i.e. creatures, see $[92,3.3(2), \S 6.3])$.

We may consider $\overline{\mathfrak{c}}=\left\langle\overline{\mathfrak{c}}_{\zeta}: \zeta<\kappa\right\rangle, \mathfrak{c}_{\zeta}$ a $\theta_{\zeta}$-creature and for some club $E$ of $\kappa, \zeta \in E \& \xi \geq \zeta \Rightarrow \theta_{\xi}>\zeta$, and consider

$\mathbb{Q}_{\overline{\mathfrak{c}}}^{2}=\left\{(w, \bar{r}):\right.$ for some $\left.\zeta, w \in \prod_{\varepsilon<\zeta} \operatorname{pos}^{\mathfrak{c}_{\varepsilon}}, \bar{r}=\left\langle r_{\varepsilon}: \varepsilon \in[\zeta, \kappa]\right\rangle, r_{\varepsilon} \in R^{\mathfrak{c}_{\varepsilon}}\right\}$ with the natural order. If $\zeta<\kappa \Rightarrow\left|R^{\mathfrak{c}_{\zeta}}\right|+\left|\operatorname{pos}^{\mathfrak{c}_{\zeta}}\right|<\kappa$ and

$(\alpha) \kappa=\sup \left\{\zeta:\right.$ for $\xi \in[\zeta, \kappa), R^{\mathfrak{c}_{\xi}}$ is $\left|\prod_{\varepsilon<\zeta} \operatorname{pos}^{\mathfrak{c}_{\varepsilon}}\right|^{+}$-complete $\}$, or

$(\beta) \nabla_{\kappa}$ 
then forcing does not collapse $\kappa^{+}$and is $(<\kappa)$-complete but the "read continuously" is problematic for case $(\beta)$.

Again, we may allow the forcing notion to be omittory (see [92, 2.1.1]), i.e., allow

$$
\begin{array}{r}
\mathbb{Q}_{\overline{\mathfrak{c}}}^{3}=\{(w, \bar{r}): \text { for some bounded } u \subseteq \kappa \text { and } A \in D, \\
\left.\qquad w \in \prod_{\varepsilon \in u} \operatorname{pos}^{\mathfrak{c}_{\varepsilon}}, \text { and } \bar{r}=\left\langle r_{\alpha}: \alpha \in A\right\rangle\right\}
\end{array}
$$

and/or a combination of creatures (i.e., the function $\Sigma$ ) and/or we may allow memory ( $=$ the object which $\mathfrak{c}_{\alpha}$ produces depends on the earlier $\mathfrak{c}_{\beta}$ 's) and/or gluing.

Note that natural nice enough c's make us regain "read continuously" and its parallels.

All those generalize naturally. But if in $\mathbb{Q}_{\left(T^{*}, \bar{c}, \theta\right), D^{*}}^{1}$ we allow $D^{*}$ to be the co-bounded filter, the proofs fail. There is more to be said and done.

Of course, we can now carry out generalizations of various independence results on cardinal invariants, e.g. between variants of $(f, g)$-bounding and slaloms (= corsets) (see [162], [176, Ch. VI, §2], Goldstern and Shelah [48], Rosłanowski and Shelah [88]). We may consider in the tree version for every limit $\delta \in S(S \subseteq \kappa$ is a stationary set) to omit tops for one or just $\leq|\delta|$ branches in a condition $p=(T, \bar{r})$, provided for each $\eta \in T,\langle|\{\varrho: \eta \triangleleft \varrho$ $\in T, \lg (\varrho)=\lg (\eta)+\alpha\}|: \alpha<\kappa\rangle$ goes to $\kappa$ and we are assuming $S$ does not reflect or at least for every strongly inaccessible $\mu<\kappa, \mu=\sup (S \cap \mu) \Rightarrow$ $\diamond S \cap \mu$ fails.

Recall that whereas Cohen forcing and many others have $\kappa$-parallels, it is not so with random real forcing.

Problem 4.33. (1) Prove that there is no reasonable parallel, say no $\kappa^{+}$-c.c. forcing notion such that any new member of ${ }^{\kappa} \kappa$ is bounded by some old one.

(2) Similarly as far as the parallel of [168] is concerned.

Concerning nep forcing notions, we may define when " $\mathbb{Q}$ is semi-nep" as follows: for some nep forcing notion $\mathbb{Q}^{*}$ we have

(a) $\mathbb{Q} \subseteq \mathbb{Q}^{*}$ (that is, the set of elements of $\mathbb{Q}$ is a subset of the set of elements of $\mathbb{Q}^{*}$ and the order of $\mathbb{Q}$ is the order of $\mathbb{Q}^{*}$ restricted to $\mathbb{Q}$ ),

(b) if $N$ is a $\mathbb{Q}$-candidate, $\mathbb{S} \in N, \mathbb{S}^{N}=\mathbb{Q}^{*} \cap N, p \in \mathbb{S}, p \in N$ then for some $q$ with $p \leq q, q$ is $(N, \mathbb{S}$ )-generic (in an explicit way).

QUESTION 4.34. Investigate "semi-nep".

5. To prove or to force, this is the question. On many things we confidently "know" that they are independent, "just" a proof is needed (for many others we know that forcing will not help by absoluteness). The rest we may think are decidable, but actually we are not sure. More fall 
in the middle; our intuitions do not give an answer, or worse: they give an answer which oscillates in time. In the mid-seventies, I was interested in (see Abraham and Shelah [3]):

Question 5.1. Is there an Aronszajn tree $T$ and a function $c: T \rightarrow$ \{red, green\} (or more colours) such that for any uncountable set $A \subseteq T$, all colours appear on the set $\{\eta \cap \nu: \eta, \nu \in A$ are incomparable $\}$, where $\eta \cap \nu$ is the maximal common lower bound?

Why? Baumgartner [11] proved that among uncountable real linear orders (e.g., with density $\aleph_{0}$ ) there may be a minimal one under embeddability. This follows from

$\mathrm{CON}$ (if $A, B \in[\mathbb{R}]^{\aleph_{1}}$ are $\aleph_{1}$-dense, then they are isomorphic).

So we may ask: among uncountable linear orders, can there be finitely many such that any other embeds one of them? (call such a family a base).

A base should contain a real order and $\omega_{1}$ and $\omega_{1}^{*}$. Any linear order into which none of them embeds is necessarily a Specker order (= take an Aronszajn tree, order it lexicographically).

You may ask: Can there be a "minimal" order among those? But there cannot. It is known ([134], answering a question of Countryman) that there is a Specker order $L$ such that the product $L \times L$ (with the product order) is the union of countably many chains (comes from a very special Aronszajn tree). Hence $L$ and $L^{*}$ (its inverse) embed no common uncountable chain. Now, consistently any two candidates for $L$ are isomorphic or antiisomorphic (see [3]). So, if we can also put the additional forcing together, then we will have a candidate for a basis which seems extremely likely. We just need to start with a Specker order, i.e., an Aronszajn tree with a lexicographic order, under the circumstances (okay to force a little), without loss of generality with any node having two immediate successors. Look at it as an Aronszajn tree, and remember Abraham and Shelah [3]. So without loss of generality, on a club the tree is isomorphic to the one for $L$, and let $\operatorname{Dom}(c)=T$,

$$
c(\eta)= \begin{cases}\text { green } & \begin{array}{l}
\text { if the two linear orders make the same } \\
\text { decision about the two immediate successors of } \eta, \\
\text { otherwise }
\end{array}\end{cases}
$$

and so question 5.1 arises.

A common property of some of the problems discussed below $(5.2,5.3$, $5.4)$ is a difference between asking about $S_{\theta}^{\lambda}=\{\delta<\lambda: \operatorname{cf}(\delta)=\theta\}$ and asking about a stationary $S \subseteq S_{\theta}^{\lambda}$ such that $S_{\theta}^{\lambda} \backslash S$ is also stationary; a 
difference of which I became aware in [136] (e.g. $\diamond_{S_{1}} \& \neg \diamond_{S_{2}}$ is possible for disjoint stationary subsets of $\omega_{1}$ ) after much agony.

QUESTION 5.2. (GCH) If $\mu$ is singular, do we have $\diamond_{S_{\mathrm{cf}(\mu)}^{\mu^{+}}}$? (Those are the only cases left.)

Similarly for inaccessibles, see [118].

If we try to force consistency of the negation, note that (for $\mu$ strong limit singular)

$$
2^{\mu}=\mu^{+}+\square_{\mu^{+}} \Rightarrow \diamond_{S_{\mathrm{cf}(\mu)}^{\mu^{+}}} \quad \text { when } \operatorname{cf}(\mu)>\aleph_{0}
$$

(see $[147,3.2$, p. 1030]). So we need large cardinals (hardly surprising for successors of singular cardinals). See more in Džamonja and Shelah [31].

Probably it is wiser to try to force this for "large" $\mu$. Changing the cofinality of a supercompact cardinal $\mu$ to $\aleph_{0}$, where " $\mu$ is prepared", is not helpful as after the forcing some old $S_{\aleph_{0}}^{\mu^{+}}$is added to the old $S_{\mu}^{\mu^{+}}$to make the new $S_{\aleph_{0}}^{\mu^{+}}$.

If $\mathbf{V}=\neg \diamond_{S_{\mu}^{\mu^{+}}}$(which may be forced but you need enough indestructibility of measure), you get $\neg{ }_{S}$ for some non-reflecting stationary $S \subseteq S_{\aleph_{0}}^{\mu^{+}}$, but we have it "cheaply" by forcing (say, starting with $\mathbf{L}$; see [147], better [119]). So maybe it is wiser to start with $\mu$ of cofinality $\aleph_{0}$.

Let $\mu$ be a limit of large cardinals and try to add enough subsets to $\mu$ to "kill" $\diamond_{\mathrm{cf}(\mu)}^{\mu^{+}}$. Our knowledge of such forcing for such cases is limited at present. But ZFC + GCH still give an approximation (see more [119]):

$(*) \quad$ if $S \subseteq S_{\operatorname{cf}(\mu)}^{\mu^{+}}$is stationary then for some $\left\langle\left\langle\alpha_{\delta, i}: i<\operatorname{cf}(\mu)\right\rangle: \delta \in S\right\rangle$ we have:

(a) $\alpha_{\delta, i}$ is increasing with limit $\delta$,

(b) if $\theta<\mu, f: \mu^{+} \rightarrow \theta$, then

$(\exists$ stationarily many $\delta \in S)(\forall i<\operatorname{cf}(\mu))\left(f\left(\alpha_{\delta, 2 i}\right)=f\left(\alpha_{\delta, 2 i+1}\right)\right)$.

Note: Having two equal values inside a group calls for division (or subtraction) so that we get a known value. So, if we are trying to guess homomorphisms from a group $G$ with $|G|=\mu^{+}$to $H,|H|=\theta<\mu, G=\bigcup_{i<\mu^{+}} G_{i}, G_{i}$ strictly increasing continuous, $\left|G_{i}\right|<\mu^{+}$, and $S \subseteq S_{\mathrm{cf}(\mu)}^{\mu^{+}}$is stationary, then we can find $\bar{\eta}=\left\langle\left\langle g_{\delta, i}: i<\operatorname{cf}(\mu)\right\rangle: \delta \in S\right\rangle$ such that $g_{\delta, i} \in G_{\alpha_{\delta, 2 i+2}} \backslash G_{\alpha_{\delta, 2 i}}$ and for every homomorphism $h: G \rightarrow H$ there are stationarily many $\delta$ such that $\left(\bigwedge_{i} h\left(g_{\delta, i}\right)=e_{H}\right)$ (relevant to Whitehead groups). Without loss of generality, $\left|G_{i+1} \backslash G_{i}\right|=\mu,\left|G_{0}\right|=\mu$, the universe of $G_{i}$ is $\mu \times(1+i)$ (or $g_{i} \in G_{i+1} \backslash G_{i}$ uses the question on $\left.f: f(i)=f\left(g_{i}\right)\right)$. See [119].

Question: Can we have something similar for any sequence $\bar{\eta}$ ? 
Answer: No. We have quite a bit of freedom (e.g., demand $\alpha_{\gamma, i} \in A^{*}$, $A^{*} \in\left[\mu^{+}\right]^{\mu^{+}}$fixed) but certainly not for any.

In fact, for any stationary non-reflecting set $S \subseteq S_{\mathrm{cf}(\mu)}^{\mu^{+}}$and any sequence $\bar{\eta}=\left\langle\left\langle\alpha_{\delta, i}: i<\operatorname{cf}(\mu)\right\rangle: \delta \in S\right\rangle$ with $\alpha_{\delta, i}$ increasing with limit $\delta$ we can force:

- for every $\left\langle h_{\delta} \in{ }^{\operatorname{cf}(\mu)} \theta: \delta \in S\right\rangle, \theta<\mu$, there is $h \in{ }^{\left(\mu^{+}\right)} \theta$ such that

$$
(\forall \delta \in S)\left(\forall^{\infty} i\right)\left(h\left(\alpha_{\delta, i}\right)=h_{\delta}(i)\right) .
$$

This is a strong negation of the earlier statements (see [147], [119]). A related ZFC result is that for a singular cardinal $\mu$, the restriction of the club filter $\mathcal{D}_{\mu^{+}}\left\lceil S_{\mathrm{cf}(\mu)}^{\mu^{+}}\right.$is not $\mu^{++}$-saturated (see Gitik and Shelah [47]).

A well known problem is

Question 5.3. (1) For a regular cardinal $\lambda>\aleph_{2}$, can $\mathcal{D}_{\lambda^{+}} \uparrow S_{\lambda}^{\lambda^{+}}$be $\lambda^{++}$-saturated?

(2) Similarly just adding the assumption GCH.

Many "club guessings" are true (see [98]), but I have looked in vain several times on:

Question 5.4. Let $\lambda$ be a regular uncountable cardinal. Can we find a sequence $\left\langle\left\langle\alpha_{\delta, i}: i\langle\lambda\rangle: \delta \in S_{\lambda}^{\lambda^{+}}\right\rangle\right.$such that $\alpha_{\delta, i}$ are increasing continuous in $i$ with limit $\delta$, and for every club $E$ of $\lambda^{+}$,

$(\exists$ stationarily many $\delta)(\exists$ stationarily many $i)\left(\alpha_{\delta, i+1}, \alpha_{\delta, i+2} \in E\right)$,

or other variants? (just $\alpha_{\delta, i+1} \in E$ is provable, $\alpha_{\delta, i} \in E$ is trivial under the circumstances).

This is interesting even under GCH, particularly as by Kojman and Shelah [71] (essentially) we deduce from it that there is a $\lambda^{+}$-Suslin tree.

We may think instead of trying to prove for $S, S_{1} \subseteq S_{\lambda}^{\lambda^{+}}$being stationary disjoint, that we can force the failure for $S$ (with GCH). This works (see [118]) but $S=S_{\lambda}^{\lambda^{+}}$is harder. The present forcing proofs fail, but also using "first counterexample" fails. We may consider proving: $\mathrm{GCH} \Rightarrow \neg \mathrm{GSH}$ (where GSH is Generalized Suslin Hypothesis: for no uncountable regular $\lambda$ is there a $\lambda$-Suslin tree). Let us look at two successor cases $\lambda^{+}, \lambda^{++}(\lambda$ regular). How can this help? Assume that there is no $\lambda^{++}$-Suslin tree and GCH holds. It follows that every stationary $S \subseteq S_{\leq \lambda}^{\lambda^{++}}$reflects in $S_{\lambda^{+}}^{\lambda^{++}}$ (see Gregory [50]), moreover it is enough to assume just that there is no $\left(<\lambda^{+}\right)$-complete Suslin tree, by Kojman and Shelah [71]. Hence 
$(\otimes)$ there is $S^{*} \subseteq \lambda^{+}$such that:

(a) (square on $\left.S^{*}\right) \bar{C}=\left\langle C_{\alpha}: \alpha \in S^{*}\right\rangle, C_{\alpha} \subseteq \alpha$ closed, otp $\left(C_{\alpha}\right) \leq \lambda$, if $\alpha$ is limit then $\sup \left(C_{\alpha}\right)=\alpha$, and $\beta \in C_{\alpha} \Rightarrow C_{\beta}=\beta \cap C_{\alpha}$,

(b) $S^{*} \cap S_{\lambda}^{\lambda^{+}}$is stationary.

(Why? This follows by [159, §4] which says that, e.g., $S_{<\lambda^{+}}^{\lambda^{++}}$is the union of $\lambda^{+}$sets with squares.) This looks as if it should help, but I have not yet found how.

Džamonja and Shelah [31] introduced

DeFinition 5.5. We say that $\lambda$ fails to weakly reflect at $\theta$ if $\theta<\lambda$ are regular uncountable cardinals and for some $F: \lambda \rightarrow \theta$ for every $\delta \in S_{\theta}^{\lambda}$ for some club $C$ of $\delta, F\lceil\delta$ is strictly increasing (equivalently, is one-to-one).

This helps to prove variants of $\mathbf{p}$ on the critical stationary subset of $\mu^{+}$ when $\mu$ is singular, i.e., on $S_{\mathrm{cf}(\mu)}^{\mu^{+}}$; see Džamonja and Shelah [31], [30], and on independence results Cummings, Džamonja and Shelah [22] and Džamonja and Shelah [29].

QUeStion 5.6. Can we get something parallel when $\operatorname{cf}(\mu)=\aleph_{0}$ ?

QUESTION 5.7. Can we prove that for some strong limit singular cardinal $\mu$ and a regular cardinal $\theta<\mu$ we have $\boldsymbol{\aleph}_{S}$, where $S=S_{\theta}^{\mu^{+}}$?

On $I[\lambda]$ see $[138],[152],[163]$. We know that e.g.

$$
\left\{\delta<\aleph_{\omega+1}: \operatorname{cf}(\delta)=\aleph_{1}\right\} \notin I\left[\aleph_{\omega+1}\right]
$$

is consistent with $\mathrm{GCH}$, but

Problem 5.8. (1) Can $\left\{\delta<\aleph_{\omega+1}: \operatorname{cf}(\delta)=\aleph_{2}\right\} \notin I\left[\aleph_{\omega+1}\right]$ ? with GCH?

(2) $\operatorname{Can}\left\{\delta<\left(2^{\aleph_{0}}\right)^{+\omega+1}: \operatorname{cf}(\delta)=\left(2^{\aleph_{0}}\right)^{+}\right\} \notin I\left[\left(2^{\aleph_{0}}\right)^{+\omega+1}\right] ?$

Now $[159, \S 4]$, dealing with successors of regulars, raises the question

QUeSTION 5.9. (1) Let $\lambda$ be inaccessible $>\aleph_{0}$. Is $I_{\lambda}^{\text {sq }}$ (see Definition 5.10 below) non-trivial, i.e., does it include stationary sets of cofinality $\sigma \in$ $\left(\aleph_{0}, \lambda\right) \cap$ Reg ? Does it include such $S$ which is large in some sense (e.g., for every such $\sigma)$ ?

(2) Similarly for successor of singular.

Definition 5.10. For a regular cardinal $\lambda>\aleph_{0}$ let

$$
\begin{aligned}
& I_{\lambda}^{\mathrm{sq}}=\left\{A \subseteq \lambda: \text { for some partial square } \bar{C}=\left\langle C_{\delta}: \delta \in S^{1}\right\rangle\right. \\
& \left.\qquad S^{1} \subseteq \lambda \text { and the set } A \backslash S^{1} \text { is not stationary in } \lambda\right\} .
\end{aligned}
$$


Definition 5.11. A linear order $I$ is $\mu$-entangled if for any pairwise distinct $t_{i, l} \in I, i<\mu, l<n$, for any $w \subseteq\{0, \ldots, n-1\}$ there are $i<j$ such that

If $|I|=\mu$ then we omit $\mu$.

$$
t_{i, l}<t_{j, l} \Leftrightarrow l \in w .
$$

QUESTION 5.12. Is there an entangled linear order of cardinality $\lambda^{+}$, where $\lambda=\lambda^{\aleph_{0}}$ ?

A "yes" answer will solve a problem of Monk [84] on the spread of ultraproducts of Boolean algebras; see [174].

With the help of pcf we can build entangled linear orders in $\lambda^{+}$for many $\lambda$, which means: provably for a proper class of $\lambda$ 's; see [166].

The interesting phenomenon is: from instances of $\mathrm{GCH}$, we can give a positive answer, but also from strong negations of $\mathrm{GCH}$ we may get a positive answer. On the one hand, if $\mu^{\aleph_{0}}=\mu, 2^{\mu}>\aleph_{\mu^{+4}}$ we have: for many $\delta<\mu^{+4}$ we get a positive answer to 5.12 with $\lambda=\aleph_{\delta}$. On the other hand, from $\mu=\mu^{\aleph_{0}}, 2^{\mu}<\mu^{+\omega}$ we can also prove a positive answer.

In fact, in the remaining case there are quite heavy restrictions on pcf. A typical universe with negative answer to 5.12 will (we think) satisfy: for a strong limit cardinal $\mu, 2^{\mu}=\mu^{+\omega+1}$, and for $\mathfrak{a} \subseteq \operatorname{Reg} \cap \mu, \mu=\sup (\mathfrak{a})$, $\operatorname{pcf}(\mathfrak{a}) \backslash \mu$ essentially concentrates on $\mu^{+\omega+1}$ (say $\left\langle\mu_{i}: i<\operatorname{cf}(\mu)\right\rangle$ increasing continuous, if $\mathfrak{a}$ is disjoint from $\left\{\mu_{i}^{+n}: i<\operatorname{cf}(\mu), 0<n<\omega\right\}$ then $\emptyset=$ $\operatorname{pcf}(\mathfrak{a}) \cap\left(\mu, \mu^{+\omega}\right)$.) See [174] and $\S 9$ here.

Maybe our knowledge of forcing will advance. Note that we need not only to have pcf structure as indicated, but also to take care of the non-pcf phenomena as well for obstructing an entangled linear order as in 5.12.

Considering a ZFC proof of existence, it seems most reasonable to assume toward a contradiction that the answer is no and consider strong limit singular $\mu$ of uncountable cofinality. So we know $2^{\mu}<\aleph_{\mu^{+4}}$ and being more careful even $2^{\mu}<\aleph_{\mu^{+}}$. Let $\gamma(*)=\min \left\{\gamma: 2^{\left(\mu^{+\gamma}\right)}>2^{\mu}\right\}$, so necessarily $\gamma(*)$ is a successor ordinal, say $\gamma(*)=\beta(*)+1$. Let $\lambda:=\mu^{+\beta(*)}$. We may consider trying to construct an entangled linear order of cardinality $\left(2^{\mu}\right)^{+}$, using the weak diamond on $\lambda^{+}=\mu^{+\beta(*)+1}$. Moreover, we know that there are trees $T$ with $\lambda^{+}$levels and $\leq \lambda^{+}$nodes and at least $\left(2^{\mu}\right)^{+}$many $\lambda^{+}$-branches (even ${ }^{\lambda^{+}} 2=\bigcup_{\zeta<2^{\mu}} \lim _{\lambda^{+}}\left(T_{\zeta}\right)$ for some subtrees $T_{\zeta}$ of ${ }^{\lambda^{+}}{ }^{2},\left|T_{\zeta}\right|=\lambda^{+}$above $)$.

Moreover, a relative of $\diamond_{\lambda^{+}}^{*}$ holds. All this seems reasonably promising, but has failed so far to solve the problem.

I have also considered to repeat the proof of the weak diamond for $\lambda^{+}$ to try to show that a tree with infinite splitting in the above representation is necessary. 
Problem 5.13. Can we prove that a stronger version of the weak diamond holds for some $\lambda$ ? E.g., a version with more than two colours and/or fixing the cofinality. We shall be glad to get even just the definable weak diamond. See [150], [102, §3], [113] and [107].

Our ignorance about such problems may well come from our gaps in forcing theory.

A major problem (more exactly a series of problems) is

Problem 5.14. (1) Can we have a reasonable theory of iterations (and/or forcing axioms) for $(<\lambda)$-complete forcing notions $\left(\lambda=\lambda^{<\lambda}\right)$ ?

(2) Similarly for forcing notions not changing cofinalities of cardinals $<\lambda$.

(3) Similarly for forcing notions preserving $\mu^{+}$and not adding bounded subsets to $\mu$, for $\mu$ a strong limit singular cardinal.

See some recent information on the first in [118], [110], [119], and even much less on the second [176, Ch. XIV], and on the third Mekler and Shelah [80], Džamonja and Shelah [28].

Though much was done on forcing for the function $2^{\lambda}$ and some specific problems, our flexibility is not as good as for $2^{\aleph_{0}}$ in forcing theory.

Particularly intriguing are solutions where we know some $\lambda$ exists but do not know which. The dual problem is iterated forcing of length Ord (class forcing); now for such iteration it is particularly hard to control in the neighborhood of singulars.

Problem 5.15. Prove the consistency of: for every $\lambda$ (or regular $\lambda$ ) a suitable forcing axiom holds. Moreover develop a theory of class forcing guaranteeing a target for every $\lambda$ (parallel to the theory of such iterations for the continuum).

Relevant is "GCH fails everywhere" (see Foreman and Woodin [36]). Now Cummings and Shelah [24], [25] is a modest try and 1.22 is relevant.

Specific well known targets are

Problem 5.16. Is GSH consistent? (Recall that GSH is the generalized Suslin hypothesis: for every regular uncountable $\lambda$ there is no $\lambda$-Suslin tree.)

PROBLEM 5.17. Is it consistent that for no regular $\lambda>\aleph_{1}$ do we have a $\lambda$-Aronszajn tree (see Abraham [1], Cummings and Foreman [23])?

A relevant problem is 6.4 . 
I have found partition theorems on trees with $\omega$ levels very useful and interesting (see Rubin and Shelah [93], and [144], [142], [176, X, XI, XV, $2.6])$. In [156, 13, p. 1453] and [137, Ch. VIII, §1] trying to prove a theorem on the number of non-isomorphic models of a pseudo elementary class we arrived at the following problem [without loss of generality, try with $2^{\lambda}=\lambda^{+}$ and see [127], by absoluteness]:

Question 5.18. Assume $m(*)<\omega, 2^{\lambda_{n}}<\lambda_{n+1}, M$ is a model with vocabulary of cardinality $\theta, \theta+\mu<\lambda_{0}, a_{\eta}^{i} \in M$ for $i<\mu, \eta \in T=$ $\bigcup_{n} \prod_{l<n} \lambda_{l}$. Can we find a strictly increasing function $h: \omega \rightarrow \omega$ and one-to-one functions $f_{n}^{i}: \prod_{k<n} \lambda_{k} \rightarrow \prod_{k<h(n)} \lambda_{k}$ such that:

(a) for $n<m$ and $\eta \in \prod_{l<m} \lambda_{l}$ we have $f_{n}^{i}\left(\eta\lceil l)=\left(f_{m}^{i}(\eta)\right)\lceil h(l)\right.$,

(b) for $n<\omega, m(*)<\omega, i_{0}<\ldots<i_{m(*)-1}<\mu$ and $\eta_{l}, \nu_{l} \in \prod_{k<n} \lambda_{k}$ for $l<m(*)$, the tuples $\left\langle a_{\eta_{0}}^{i_{0}}, a_{\eta_{1}}^{i_{1}}, \ldots, a_{\eta_{m(*)-1}}^{i_{m(*)-1}}\right\rangle,\left\langle a_{\nu_{0}}^{i_{0}}, a_{\nu_{1}}^{i_{1}}, \ldots, a_{\nu_{m(*)-1}}^{i_{m(*)-1}}\right\rangle$ realize the same type in $M$ provided that for $l, m<m(*), \lg \left(\eta_{l} \cap \eta_{m}\right)=\lg \left(v_{l} \cap v_{m}\right)$, and if so that $\left(\eta_{l}(h)<\eta_{m}(h)\right) \equiv v_{l}(h)<v_{m}(h)$ for $h=\lg \left(\eta_{l} \cap \eta_{m}\right)$ ?

(If on $\lambda_{n}$ there is a "large ideal" (see [171]) life is easier, see [156].)

6. Boolean algebras and iterated forcing. We turn to Boolean algebras. Monk has made extensive lists of problems about Boolean algebras (which inspired quite a few works of mine). His problems mostly go systematically over all possible relations; our perspective is somewhat different.

Among my results on Boolean algebras I like 6.1 stated below (see [151]), but the result did not draw much attention though the paper was noticed (see Bonnet and Monk [17], Juhász [64]).

THEOREM 6.1. If $B$ is a Boolean algebra of cardinality $\geq \beth_{\omega}$ and $\lambda=$ $\operatorname{Id}(B)$ (the number of ideals of the Boolean algebra) then $\lambda=\lambda^{\beth_{\omega}}$.

(We can instead of ideals of Boolean algebras speak about open subsets of a compact Hausdorff topology, and we can replace $\beth_{\omega}$ by any singular strong limit).

So we are left with

QUestion 6.2. Is it true that for any large enough Boolean algebra $B$ we have $\operatorname{Id}(B)=\operatorname{Id}(B)^{<\theta}$ when, e.g., $\theta=\log _{2}(|B|)$, or at least for some constant $n, \theta=\min \left\{\mu: \beth_{n}(\mu) \geq|B|\right\}$ ? (Similarly for compact spaces.)

By [151], for every $B$ there is such $n$. Of course, in non-specially constructed universes the answer is yes. If you like to try consistency, you have to use the phenomena proved consistent in Gitik and Shelah [45].

On the other hand, a ZFC proof may go in a different way than [151]. Related (see Juhász [63]) is 
QUESTION 6.3. What can be the number of open sets of a $T_{2}$ topology? $T_{3}$ topology? One with clopen basis?

It seems interesting to consider the following

Problem 6.4. Is there a class of cardinals $\lambda$ (or just two) such that there is a $\left(\lambda^{+}, \lambda\right)$-thin tall superatomic Boolean algebra $B$ (i.e., $|B|=\lambda^{+}, B$ is superatomic and for every $\alpha<\lambda^{+}, B$ has $\leq \lambda$ atoms of order $\alpha$ ), provably in ZFC?

It is well known that if $\lambda=\lambda^{<\lambda}$ then there is a $\left(\lambda^{+}, \lambda\right)$-thin tall superatomic Boolean algebra, so for $\lambda=\aleph_{0}$ there is one, so for negative consistency we need "GCH fails everywhere or at least for every large enough $\lambda^{\prime}$ ". Also note that trivially if there is a $\lambda^{+}$-tree (i.e., one with $\lambda^{+}$levels each of cardinality $\leq \lambda)$, then there is such a $\left(\lambda^{+}, \lambda\right)$-thin tall superatomic Boolean algebra.

The point is that for several problems in Monk [84]: Problems 72, 74, 75 and ZFC versions of Problems 73, 77, 78, 79 (all solved in Rosłanowski and Shelah [87] in the original version, i.e. showing consistency) there is no point to try to get positive answers as long as we do not know it for 6.4.

Also for several problems of [84] (Problems 49, 57, 58, 61, 63, 87) there is no point to try to get consistency of non-existence as long as we have not proved the consistency of the GSH (generalized Suslin hypothesis) which says there are no $\lambda^{+}$-Suslin trees or there is no $\lambda$-Suslin tree for $\lambda=\operatorname{cf}(\lambda)>$ $\aleph_{0}$. For some others this is not provable, but it still seems very advisable to wait for the resolution of GSH.

Problem 6.5. Usually the question on cardinal invariants $\mathbf{i n v}_{1}, \mathbf{i n v}_{2}$ is "do we always have $\operatorname{inv}_{1}(B) \leq \operatorname{inv}_{2}(B)$ ?", or "do we always have $2^{\operatorname{inv}_{1}(B)} \geq$ $\operatorname{inv}_{2}(B)$ ?" But maybe there are relations like $\operatorname{inv}_{1}(B) \leq\left(\operatorname{inv}_{2}(B)\right)^{+n}$ for some fixed $n<\omega$ (or for $\omega$ ). A particularly suspicious case is $|B| \leq$ $\left(\operatorname{irr}_{n}(B)\right)^{+}$, where $n \in[2, \omega]$ and

$\operatorname{irr}_{n}(B)=\sup \{|X|: X \subseteq B$ is $n$-irredundant, which means:

if $m<1+n$ and $a_{0}, \ldots, a_{m} \in X$ are pairwise distinct then $a_{0}$ is not in the subalgebra generated by

$$
\left.\left\{a_{1}, \ldots, a_{m}\right\}\right\} \text {. }
$$

(If $n=\omega$ we may omit it.)

I think that for $n=\omega$ the case $|B|=\aleph_{2}$ had appeared in an old list of Monk, but not in [84]. We may also ask $|B| \leq\left(\operatorname{irr}_{n}(B)\right)^{+m}$ for $n, m \in[2, \omega]$.

Of course, the open case is when, say, $|B|=\lambda^{++}, 2^{\lambda} \geq \lambda^{++}$. Thinking about this problem, I was sure the answer is consistently no (consistently yes is easy, even in the Easton model). Moreover, I feel I know how to do it: let $\lambda=\lambda^{<\lambda}, \lambda^{+}<\theta<\mu, \mathfrak{B}$ be a suitable algebra on $\mu$ with $\leq \lambda$ functions. 
A member $p$ of the forcing notion $\mathbb{P}$ consists of $w^{p} \in[\mu]^{<\lambda}$ and a Boolean algebra $B^{p}$ generated by $\left\{x_{i}: i \in w^{p}\right\}$ but such that

- if $B^{p} \models " x_{i_{0}}=\sigma\left(x_{i_{1}}, \ldots, x_{i_{n}}\right)$ " for ordinals $i_{0}, \ldots, i_{n} \in w^{p}$ and a Boolean term $\sigma$ then $i_{0} \in \operatorname{cl}_{\mathfrak{B}}\left(\left\{i_{1}, \ldots, i_{n}\right\}\right)$ and possibly more

(the order of $\mathbb{P}$ is the natural one). This is to reconcile the demand "the Boolean algebra has cardinality $\mu$, so without loss of generality we have to ask $i<j \Rightarrow x_{i} \neq x_{j}$ " and the $\lambda^{+}$-c.c. Of course, $\underset{\sim}{B}=\bigcup\left\{B^{p}: p \in G_{\mathbb{P}}\right\}$.

Note: if $\lambda=\aleph_{0}$ we have more freedom. (The expected proof goes: if

$\Vdash$ " $\underset{\sim}{X} \subseteq \underset{\sim}{B}, \underset{\sim}{X}=\left\{\underline{\sim}_{i}: i<\theta\right\}$ with no repetition

$$
\text { exemplifies } \operatorname{irr}(\underset{\sim}{B})^{+} \geq|B| "
$$

then we can find $p_{i}, p \leq p_{i} \in \mathbb{P}, p_{i} \Vdash$ " $y_{i}=\sigma_{i}\left(x_{\alpha(i, 0)}, \ldots, x_{\alpha\left(i, n_{i}\right)}\right)$ ". Hence, if $(\forall \alpha<\theta)\left(|\alpha|^{<\lambda}<\theta=\operatorname{cf}(\theta)\right)$, without loss of generality, $\sigma_{i}=\sigma, n_{i}=n^{*}$ and $\left\langle\left(p_{i},\left\langle\alpha(i, l): l<n^{*}\right\rangle\right): i<\theta\right\rangle$ forms a $\Delta$-system (moreover indiscernible as a sequence of sequences of ordinals of length $<\lambda)$. Let $\left\{\alpha(i, l): l<n^{*}\right\} \subseteq$ $w^{p}=w^{*} \cup\left\{\gamma_{i, \zeta}: \zeta<\zeta^{*}\right\}, \zeta^{*}<\lambda$, etc., and we have to find $n<\omega_{1}, i_{0}<\ldots<$ $i_{n}<\theta$ and $q$ above $p_{i_{0}}, \ldots, p_{i_{n}}$ such that $B^{q}=" y_{i_{0}}=\sigma^{*}\left(y_{i_{1}}, \ldots, y_{i_{n}}\right)$ ". So it is natural to demand $\zeta<\zeta^{*} \Rightarrow \gamma_{i_{0}, \zeta} \in \operatorname{cl}_{\mathfrak{B}}\left\{\gamma_{i_{1}, \zeta}, \ldots, \gamma_{i_{n}, \zeta}\right\}$. So if $\lambda=\aleph_{0}$ we may use $n>\left|w^{p_{i}}\right|$. But this approach has not converged to a proof.)

So (see Monk [84, Problem 28])

Question 6.6. (1) Is there a class of (or just one) $\lambda$ such that for some Boolean algebra $B$ of cardinality $\lambda^{+}$we have $\operatorname{irr}(B)=\lambda$ ?

(2) Similarly for $\operatorname{irr}_{n}(B)$.

Colouring theorems (e.g. [172]) are not enough for a construction.

Question 6.7. (1) For which pairs $(\lambda, \theta)$ of cardinals $\lambda \geq \theta$ is there a superatomic Boolean algebra $B$ with $>\lambda$ elements, $\lambda$ atoms and such that every $f \in \operatorname{Aut}(B)$ moves $<\theta$ atoms? (That is, $\mid\{x: B \mid=$ " $x$ an atom and $f(x) \neq x "\} \mid<\theta$.)

(2) In particular, is it true that for some $\theta$, for a proper class of $\lambda$ 's there is such a Boolean algebra?

(3) Replace "automorphism" by "one-to-one endomorphism".

See some results in $[104, \S 1, \S 2]$ for $\theta$ strong limit singular. (It may be interesting to try: with $n$ depending on the arity of the term as in [104].)

Concerning attainment in ZFC:

Question 6.8. (1) Can we show the distinction made between the attainments of variants of hL (and hd), in a semi-ZFC way? That is, in Rosłanowski and Shelah [86] such examples are forced. "Semi-ZFC" means can we prove such examples exist after adding to ZFC only restrictions on cardinal arithmetic? 
(2) Similarly for other consistency results. (Well, preferably of low consistency strength.)

In view of [103], [127] it is reasonable to consider

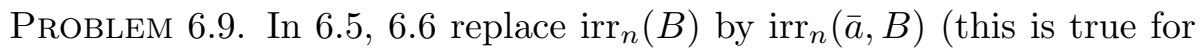
other cardinal invariants as well); see Definition 6.10 below.

Definition 6.10. Let $B$ be a Boolean algebra and $\bar{a}=\left\langle a_{i}: i<\lambda\right\rangle$ be a sequence of elements of $B$.

(1) $\operatorname{irr}_{n}(\bar{a}, B)=\sup \left\{|X|: X \subseteq \lambda\right.$ and $\left\langle a_{i}: i \in X\right\rangle$ is $n$-irredundant $\}$.

(2) Similarly for other invariants of the "universal family" from Rosłanowski and Shelah [91] (see Definition 1.1 there).

QUESTION 6.11. Is there (at least consistently) a Boolean algebra $B^{*}$ such that if $B$ is a Boolean algebra extending $B^{*}$ then for some ultrafilters $D_{1}, D_{2}$ on $B$ we have: $\left(B, D_{1}\right),\left(B, D_{2}\right)$ are not isomorphic, i.e., no automorphism of $B$ maps $D_{1}$ onto $D_{2}$ ?

A close topological relative is: "is there a homogeneous compact Hausdorff space of cellularity $>2^{\aleph_{0}}$ ?" (van Douwen, see Kunen [75]).

There are some lemmas in [151] which help to prove 6.1, and I would like to know whether the bounds used there are the best possible. Those lemmas also show that for some cardinal invariants (for Boolean algebras or topologies), defined by supremum, if the supremum is not attained, then the value is "almost" regular (the classical result of Erdoss and Tarski on the cellularity of a Boolean algebra (or topology) says it is regular, whereas we prove in [151] that, e.g., the spread satisfies $2^{\mathrm{cf}\left(\mathrm{s}^{+}(B)\right)}>\mathrm{s}^{+}(B)$ for a Boolean

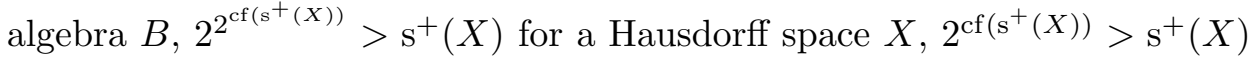
for a $T_{3}$ space $\left.X\right)$.

Question 6.12. Can we find more applications of the theorems (and proofs) in [151] implying (or saying) that if the supremum in some cardinal invariants for a space $\mathcal{U}$ (or a Boolean algebra $B$, or whatever) is not attained, then it has large cofinality?

A recent application is in Rosłanowski and Shelah $[87, \S 6]$. This is a converse to 6.8 .

For the spread (and the hereditarily Lindelöf degree and the hereditarily density) the results are best possible (see Juhász and Shelah [65]), and for regular spaces we have better results $([151,5.1]$ also best possible) but are the bounds in the claims below best possible? 
Definition 6.13. (1) $\varphi$ is nice for $X$ if $\varphi$ is a function from the family of subsets of the topological space $X$ to cardinals satisfying

$$
\varphi(A) \leq \varphi(A \cup B) \leq \varphi(A)+\varphi(B)+\aleph_{0}
$$

(i.e., monotonicity and subadditivity).

(2) We say $\varphi$ is $(\chi, \mu)$-complete provided that if $A_{i} \subseteq X, \varphi\left(A_{i}\right)<\chi$ for $i<\mu$ then $\varphi\left(\bigcup_{i<\mu} A_{i}\right)<\chi$. Let $C(\varphi, \mu)=\{\chi: \varphi$ is $(\chi, \mu)$-complete $\}$.

(3) We say $\varphi$ is $(<\lambda, \mu)$-complete if for arbitrarily large $\chi<\lambda, \varphi$ is $(\chi, \mu)$-complete.

(4) Let $\mathrm{Ch}_{\varphi}$ be the following function from $X$ to cardinals:

$$
\mathrm{Ch}_{\varphi}(y)=\min \{\varphi(u): y \in u \in \tau(X)\},
$$

where $\tau(X)$ is the topology of $X$, that is, the family of open sets.

REMARK 6.14. (1) We can replace $\mu$ by $<\mu$ and $i<\mu$ by $i<\alpha<\mu$, and make suitable changes later.

(2) In our applications we can restrict the domain of $\varphi$ to the Boolean algebra generated by $\tau(X)$ and even more, e.g., in $6.15,6.17$ below to simple combinations of the $u_{i, \xi, \zeta}$.

(3) We can change the definition of $(<\lambda, \mu)$-complete to

- if $A_{i} \subseteq X$ (for $i<\mu$ ) and $\sup _{i<\mu} \varphi\left(A_{i}\right)<\lambda$ then $\varphi\left(\bigcup_{i<\mu} A_{i}\right)<\lambda$, without changing our subsequent use. [We will then use: if $\varphi\left(A_{\alpha}\right)<\chi_{i}$ for $\alpha<\mu$ then $\varphi\left(\bigcup_{\alpha<\mu} A_{\alpha}\right)<\chi_{i+1}$.]

Lemma 6.15. Suppose $\lambda$ is a singular cardinal of cofinality $\theta, \lambda=$ $\sum_{i<\theta} \chi_{i}, \chi_{i}<\lambda, \theta<\lambda$ and $\mu=\beth_{5}(\theta)^{+}$, or even just $\mu=\beth_{2}\left(\beth_{2}(\theta)^{+}\right)^{+}$. Assume that:

(i) $\varphi$ is nice for $X$,

(ii) $X_{\chi_{i}}=\left\{y \in X: \mathrm{Ch}_{\varphi}(y) \geq \chi_{i}\right\}$ has cardinality $\geq \mu$ for $i<\theta$,

(iii) $\varphi$ is $(<\lambda, \mu)$-complete.

Then there are open sets $u_{i} \subseteq X($ for $i<\theta)$ such that

$$
\varphi\left(u_{i} \backslash \bigcup_{j \neq i} u_{j}\right) \geq \chi_{i} .
$$

REMARK 6.16. If $\left|\left\{y \in X: \mathrm{Ch}_{\varphi}(y) \geq \chi_{i}\right\}\right|<\mu$, it essentially follows from $\left(\chi_{i}, \mu\right)$-completeness that $\varphi\left(X_{\chi_{i}}\right) \geq \lambda$, where $X_{\chi}=\bigcup\{u \in \tau(X)$ : $\varphi(u)<\chi\}$. Otherwise, $\varphi\left(X \backslash X_{\chi_{i}}\right) \geq \lambda$ by subadditivity, but $\varphi\left(X \backslash X_{\chi_{i}}\right) \leq$ $\prod\left\{\varphi(\{y\}): y \in X \backslash X_{\chi_{i}}\right\}$, so by $\left(\chi_{i}, \mu\right)$-completeness $\varphi(\{y\}) \geq \chi_{i}$ for some $y \in X$, which is impossible for the instances which interest us.

LEMMA 6.17. Suppose that $X$ is a Hausdorff space, $\lambda$ is a singular cardinal, $\theta=\operatorname{cf}(\lambda), \lambda=\sum_{i<\theta} \chi_{i}, \chi_{i}<\lambda, \mu<\lambda$ and clauses (i)-(iii) of 6.15 hold $($ for $\varphi)$. 
(1) If $\mu=\beth_{2}(\theta)^{+}$(or even $\left.\sum_{\sigma<\theta} \beth_{2}(\sigma)^{+}\right)$, then there are open sets $u_{i}$ (for $i<\theta)$ such that $\varphi\left(u_{i} \backslash \bigcup_{j>i} u_{j}\right) \geq \chi_{i}$.

(2) If $X=\bigcup\{u: \varphi(u)<\lambda\}$, and $\mu$ is as in part (1), then there are open sets $u_{i}($ for $i<\theta)$ such that $\varphi\left(u_{i} \backslash \bigcup_{j \neq i} u_{j}\right) \geq \chi_{i}$.

(3) If $\mu \geq \beth_{3}\left(2^{<\theta}\right)^{+}, \varphi$ is $\left(<\chi_{0}, \mu\right)$-complete, then there are $u_{i}($ for $i<\theta)$ such that $\varphi\left(u_{i} \backslash \bigcup_{j \neq i} u_{j}\right) \geq \chi_{0}\left(\right.$ so $\lambda, \chi_{i}(0<i<\theta)$ are irrelevant $)$.

Remark 6.18. Part (1) of the lemma is suitable to deal with Boolean algebras, part (2) with the existence of $\left\{x_{\alpha}: \alpha<\lambda\right\}$ such that for every $\alpha<\lambda$, for some $u, x_{\alpha} \in u \cap\left\{x_{\beta}: \beta<\lambda\right\} \subseteq\left\{x_{\beta}: \beta \leq \alpha\right\}$.

Now,

Question 6.19. Are the cardinal bounds in $6.14-6.17$ best possible?

7. A taste of algebra. I have much interest in Abelian groups, but better see Eklof and Mekler [32].

Thomas prefers to deal just with short elegant proofs of short elegant problems (for me the second demand suffices). So he was rightly happy when proving that for any infinite group $G$ with no centre, $\gamma(G)<\left(2^{|G|}\right)^{+}$, where $G^{[0]}=G, G^{[1]}$ is the automorphism group of $G$ considered as an extension of $G, G^{[i+1]}=\left(G^{[i]}\right)^{[1]}, G^{[\delta]}=\bigcup_{i<\delta} G^{[i]}$, so $G^{[i]}$ is an increasing sequence of groups with no centre, and $\gamma(G)=\min \left\{\gamma: G^{[\gamma]}=G^{[\gamma+1]}\right\}$.

But is there a better cardinal bound? No, for $|G|$ regular $>\aleph_{0}$, see Just, Shelah and Thomas [67], but we are left with:

QUESTION 7.1. If $G$ is a countable group with a trivial centre, then do we have $\gamma(G)<\omega_{1}$ ? What about singular $|G|$ ?

I heard about the following problem (see Hamkins [56]).

Problem 7.2. If $G$ is a group possibly with centre, $G^{[i]}$ is defined as above but we have just a homomorphism $h_{i+1, i}: G^{[i]} \rightarrow G^{[i+1]}$ with the centre of $G^{[i]}$ being the kernel (and in limit stages take the direct limit), is there a bound on $\gamma(G)$ really better than the first strongly inaccessible $>|G|$ (obtained by Hamkins [56])?

Thomas had also started investigating cofinalities of some natural groups (see Sharp and Thomas [95], [96], Thomas [193]). He drew me to it and I was particularly glad to see that pcf pops in naturally; e.g. (see Shelah and Thomas [186]) if $\lambda_{n} \in \operatorname{CF}(\operatorname{Sym}(\omega))$ and $\lambda \in \operatorname{pcf}\left\{\lambda_{n}: n<\omega\right\}$ then $\lambda \in \mathrm{CF}(\operatorname{Sym}(\omega))$, where

Definition 7.3. (1) $\mathrm{CF}(G)=\{\theta: \theta=\operatorname{cf}(\theta)$ and there is an increasing sequence of proper subgroups of $G$ of length $\theta$ with union $G\}$.

(2) $\operatorname{cf}(G)=\min \left[\mathrm{CF}(G) \backslash\left\{\aleph_{0}\right\}\right]$. 
Though we found some information about $\operatorname{cf}\left(\prod_{n}\right.$ alt $\left.(n)\right)$ (see Saxl, Shelah and Thomas [180], where $\operatorname{alt}(n)$ is the group of even permutations of $\{0, \ldots, n-1\})$, we remained baffled by

QUESTION 7.4. Is it consistent that $\aleph_{2} \leq \operatorname{cf}\left(\prod_{n<\omega}\right.$ alt $\left.(n)\right) ?$

It is natural to try to use iterations of length $\omega_{2}$, where each iterand consists of trees with norms (see Rosłanowski and Shelah [92]). Naturally, a norm on $\mathcal{P}(\operatorname{alt}(n))$ will be such that if $\operatorname{nor}(A) \geq m+1$ and $\sigma$ is a group term, then we can have for "many" $f_{1}, \ldots, f_{k}, g \in \operatorname{alt}(n)$ that $A^{\prime}=\{h \in$ $\left.A: \sigma\left(h, f_{1}, \ldots, f_{k^{*}}\right)=g\right\}$ has $\operatorname{nor}\left(A^{\prime}\right) \geq m$ toward destroying a guess on an approximation to a lower subgroup exemplifying $\omega_{1} \in \mathrm{CF}\left(\prod_{n<\omega}\right.$ alt $\left.(n)\right)$. This helps for $\aleph_{0} \notin \mathrm{CF}\left(\prod_{n<\omega}\right.$ alt $\left.(n)\right)$, but fails for the purpose of 7.4 .

My interest in lifting for the measure algebra started when Talagrand promised me "flowers on your grave from every measure theorist" (a little hard for me to check), and Fremlin said essentially the same, conventionally (see [145], [176, Ch. IV]). But this does not solve some other problems from Fremlin's list, of which I particularly like

Question 7.5. Assume CH (or even GCH or just prove consistency). Do we have lifting for every measure algebra? Which means: let $\mathcal{B}(I)$ be the algebra of subsets of ${ }^{I} 2$ generated from clopen ones by countable unions and intersections, $\mu_{\mathcal{B}}$ the Lebesgue measure on $\mathcal{B}$ (so we get the so-called Maharam algebra), $\mathbf{I}=\left\{A \in \mathcal{B}: \mu_{\mathcal{B}}(A)=0\right\}$ (so $\mathbf{I}$ is the ideal of null sets). A lifting is a homomorphism from $\mathcal{B} / \mathbf{I}$ into $\mathcal{B}$ such that

$$
X \in \mathcal{B} \Rightarrow f(X / \mathbf{I})=X \bmod \mathbf{I} \text {. }
$$

Naturally, we think $I_{0} \subseteq I_{1} \Rightarrow \mathcal{B}\left(I_{0}\right) \subseteq \mathcal{B}\left(I_{1}\right)$ (by identifying) and for an increasing sequence $\bar{I}=\left\langle I_{\alpha}: \alpha<\alpha^{*}\right\rangle$ we let $\mathcal{B}(\bar{I})=\bigcup_{\alpha<\alpha^{*}} \mathcal{B}\left(I_{\alpha}\right)$. In the positive direction we may try to prove by induction on $\lambda$; then we will be naturally drawn to proving: for any $\mathcal{P}^{-}(n)$-diagram $\left\langle\mathcal{B}\left(\bar{I}_{s}\right): s \in \mathcal{P}^{-}(n)\right\rangle$, where $\mathcal{P}^{-}(A)=\{u: u \subseteq A, u \neq A\}$, and a sequence of liftings $\bar{f}=\left\langle f_{s}: s \in\right.$ $\left.\mathcal{P}^{-}(n)\right\rangle$ satisfying a reasonable induction hypothesis, $\bigcup_{s} f_{s}$ can be extended to a lifting of $\bigcup_{s} I_{s, \alpha}$ (as e.g. in [143], Sageev and Shelah [94], but we have to notice the non-continuity for limit of cofinality $\aleph_{0}$ ). For the negative direction we may think of using a partition theorem.

For a long time I have been interested in compactness in singular cardinals; i.e., whether if something occurs for "many" subsets of a singular $\lambda$ of 
cardinality $<\lambda$, it occurs for $\lambda$. For the positive side (on the filters see 7.7 below) we have

TheOREM 7.6. Let $\lambda$ be a singular cardinal, $\chi^{*}<\lambda$. Assume that $\mathbf{F}$ is a set of pairs $(A, B)$ (written usually as $B / A ; \mathbf{F}$ stands for free) with $A, B \subseteq \mathcal{U}$ satisfying axioms $I I, I I I, I V, V I, V I I$ below. Let $A^{*}, B^{*} \subseteq \mathcal{U}$ with $\left|A^{*}\right|=\lambda$. Then $B^{*} / A^{*} \in \mathbf{F}$ if $B^{*} / A^{*}$ is $\lambda$-free in a weak sense, which means (see Definition 7.7 below):

$(*)_{0} \quad$ for the $\mathcal{D}_{\chi^{*}}\left(B^{*}\right)$-majority of $B \in\left[B^{*}\right]<\lambda$ we have $B / A^{*} \in \mathbf{F}$, or just

$(*)_{1} \quad$ the set $\left\{\mu<\lambda:\left\{B \in\left[B^{*}\right]^{\mu}: B / A^{*} \in \mathbf{F}\right\} \in \mathcal{E}_{\mu}^{\mu^{+}}\left(B^{*}\right)\right\}$ contains a club of $\lambda$, or at least

$(*)_{2} \quad$ for some set $C$ of cardinals $<\lambda$, unbounded in $\lambda$ and closed (meaningful only if $\left.\operatorname{cf}(\lambda)>\aleph_{0}\right)$, for every $\mu \in C$, for an $\mathcal{E}_{\mu}^{\mu^{+}}\left(B^{*}\right)$-positive set of $B \in\left[B^{*}\right]^{\mu}$ we have $B / A^{*} \in \mathbf{F}$.

The axioms are:

Ax. II: $B / A \in \mathbf{F} \Leftrightarrow A \cup B / A \in \mathbf{F}$,

Ax. III: if $A \subseteq B \subseteq C, B / A \in \mathbf{F}$ and $C / B \in \mathbf{F}$ then $C / A \in \mathbf{F}$,

Ax. IV: if $\left\langle A_{i}: i \leq \theta\right\rangle$ is increasing continuous, $\theta=\operatorname{cf}(\theta), A_{i+1} / A_{i} \in \mathbf{F}$ then $A_{\theta} / A_{0} \in \mathbf{F}$,

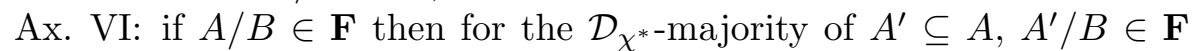
(see below),

Ax. VII: if $A / B \in \mathbf{F}$ then for the $\mathcal{D}_{\chi^{*}-m a j o r i t y ~ o f ~} A^{\prime} \subseteq A, A / B \cup A^{\prime} \in \mathbf{F}$.

(Of course we can get variants by putting more or less into the statement.)

Definition 7.7. (1) Let $\mathcal{D}$ be a function giving for any set $B^{*}$ a filter $\mathcal{D}\left(B^{*}\right)$ on $\mathcal{P}\left(B^{*}\right)$ (or on $\left.\left[B^{*}\right]^{\mu}\right)$. Then to say "for the $\mathcal{D}$-majority of $B \subseteq B^{*}$ (or $B \in\left[B^{*}\right]^{\mu}$ ) we have $\varphi(B)$ " means $\left\{B \subseteq B^{*}: \varphi(B)\right\} \in \mathcal{D}\left(B^{*}\right)$ (or $\left.\left\{B \in\left[B^{*}\right]^{\mu}: \neg \varphi(B)\right\}=\emptyset \bmod \mathcal{D}\right)$.

(2) Let $\mathcal{D}_{\mu}\left(B^{*}\right)$ be the family of $Y \subseteq \mathcal{P}\left(B^{*}\right)$ such that for some algebra $M$ with universe $B^{*}$ and $\leq \mu$ functions,

$Y \supseteq\left\{B \subseteq B^{*}: B \neq \emptyset\right.$ is closed under the functions of $\left.M\right\}$.

(3) $\mathcal{E}_{\mu}^{\mu^{+}}\left(B^{*}\right)$ is the collection of all $Y \subseteq\left[B^{*}\right]^{\mu}$ such that: for some $\chi, x$ such that $\left\{B^{*}, x\right\} \in \mathcal{H}(\chi)$, if $\bar{M}=\left\langle M_{i}: i<\mu^{+}\right\rangle$is an increasing continuous sequence of elementary submodels of $(\mathcal{H}(\chi), \in)$ such that $x \in M_{0}$ and $\bar{M} \uparrow(i+1) \in M_{i+1}$, then for some club $C$ of $\mu^{+}, i \in C \Rightarrow M_{i} \cap B^{*} \in Y$.

On the filters see Kueker [73], and [133, §3]. The theorem was proved in [133] but with two extra axioms, however it included the full case of varieties (i.e., including the non-Schreier ones). Later, the author eliminated those two extra axioms: Ax. V in Ben David [14], and Ax. I in [135] (answering a 
question of Fleissner on providing a combinatorial proof of the compactness). Hodges [58] also contains relatives of this result.

There are some cases of incompactness (see Fleissner and Shelah [35], and [157]).

Problem 7.8. (1) Are there general theorems covering the incompactness phenomena?

(2) Are there significantly better compactness theorems (for uncountable cofinality, of course)?

\section{Related is}

Question 7.9. What can be

$\{\lambda$ : there is an algebra $M$ of cardinality $\lambda, \lambda$-free for $\mathcal{V}$ but not free $\}$, for a variety $\mathcal{V}$ (at least with countable vocabulary)? (See Eklof and Mekler [32], Mekler and Shelah [81], Mekler, Shelah and Spinas [82].)

There are cases of strong dichotomy: if $\geq \lambda$ then $\geq 2^{\lambda}$, related to groups (see [153], Grossberg and Shelah [51], [52], and [129]; on Abelian groups see Fuchs [39]).

Question 7.10. [V $=\mathbf{L}]$ If $\lambda>\operatorname{cf}(\lambda)>\aleph_{0}, G$ is a torsion free Abelian group of cardinality $\lambda$, can $\lambda=\nu_{p}(\operatorname{Ext}(G, \mathbb{Z})) ?$

The cardinal $\nu_{p}(\operatorname{Ext}(G, \mathbb{Z}))$ is the dimension of $\{x \in \operatorname{Ext}(G, \mathbb{Z}): p x=0\}$ as a vector space over $\mathbb{Z} / p \mathbb{Z}$. To avoid Ext note that (see Fuchs [39]) this group can be represented as $\operatorname{Hom}(G, \mathbb{Z} / p \mathbb{Z}) /\{h / p: h \in \operatorname{Hom}(G, \mathbb{Z})\}$. If $G$ is torsion free, then the group $\operatorname{Ext}(G, \mathbb{Z})$ is divisible and hence the ranks $\nu_{p}(\operatorname{Ext}(G, \mathbb{Z}))$ for $p$ prime, and $\nu_{0}(\operatorname{Ext}(G, \mathbb{Z}))$, the rank of $\{x \in \operatorname{Ext}(G, \mathbb{Z})$ : $x$ torsion $\}$, determine $\operatorname{Ext}(G, \mathbb{Z})$ up to isomorphism. If we assume $(\mathbf{V}=\mathbf{L}$ and) there is no weakly compact cardinal, this question is the only piece left for characterizing the possible such $\operatorname{Ext}(G, \mathbb{Z})$ (see Mekler, Rosłanowski and Shelah [79]).

QUESTION 7.11. What is the first cardinal $\lambda=\lambda_{\kappa}$ such that: for every ring $R$ of cardinality $\leq \kappa$, if there is an endorigid (or rigid, or 1-to-1 rigid) $R$-module of size $\geq \lambda$, then there are such $R$-modules in arbitrarily large cardinals? (i.e. Hanf numbers).

8. Partitions and colourings. Remember (on the partition "arrow" see Erdős, Hajnal, Maté and Rado [34])

Definition 8.1. (1) $\lambda \rightarrow(\alpha)_{\kappa}^{n}$ means: for every colouring $c:[\lambda]^{n} \rightarrow \kappa$ there is a set $X \subseteq \lambda$ of order type $\alpha$ such that $c \uparrow[X]^{n}$ is constant. 
(2) $\lambda \rightarrow[\alpha]_{\kappa}^{n}$ means: for every colouring $c:[\lambda]^{n} \rightarrow \kappa$ there is a set $X \subseteq \lambda$ of order type $\alpha$ such that $\operatorname{Rang}\left(c \uparrow[X]^{n}\right) \neq \kappa$.

(3) $\lambda \rightarrow[\alpha]_{\kappa, \sigma}^{n}$ means: for every colouring $c:[\lambda]^{n} \rightarrow \kappa$ there is a set $X \subseteq \lambda$ of order type $\alpha$ such that $\operatorname{Rang}\left(c \uparrow[X]^{n}\right)$ has cardinality $<\sigma$.

Definition 8.2. $M$ is a Jonsson algebra if it is an algebra with countably many functions with no proper subalgebra of the same cardinality. (See [166], [98].)

Definition 8.3. $\operatorname{Pr}_{1}(\lambda, \mu, \theta, \sigma)$ means: there is $c:[\lambda]^{2} \rightarrow \theta$ such that if $u_{i} \in[\lambda]^{<\sigma}$ (for $i<\mu$ ) are pairwise disjoint and $\gamma<\theta$ then for some $i<j<\mu, c \uparrow\left(u_{i} \times u_{j}\right)$ is constantly $\gamma$. (See [166], [98].)

There are many more variants.

It irritates me that after many approximations, I still do not know (better for me "consistently no", better for set theory "yes") the answer to the following.

QUESTION 8.4. If $\mu$ is singular, is there a Jonsson algebra on $\mu^{+}$? (and even better $\operatorname{Pr}_{1}\left(\mu^{+}, \mu^{+}, \mu^{+}, \operatorname{cf}(\mu)\right)$ ?)

Also the requirements on an inaccessible to get colouring theorems may well be an artifact of my inability, so let us state minimal open cases.

Question 8.5. (1) Let $\lambda$ be the first $\omega$-Mahlo cardinal. Does $\lambda \nrightarrow[\lambda]_{\lambda}^{2}$ or at least $\lambda \nrightarrow[\lambda]_{\theta}^{2}$ for $\theta<\lambda$ ?

(2) Let $\lambda$ be the first inaccessible cardinal which is $(\lambda \cdot \omega)$-Mahlo. Is there a Jonsson algebra on $\lambda$ ? (Even better $\lambda \nrightarrow[\lambda]_{\lambda}^{2}$ ?)

In both parts it is better to have $\operatorname{Pr}_{1}\left(\lambda, \lambda, \lambda, \aleph_{0}\right)$, etc.; it is interesting even assuming GCH.

Whereas under GCH the relation $\rightarrow$ for cardinals is essentially understood (see Erdős, Hajnal, Maté and Rado [34]), the case of ordinals is not. As by [132] (GCH for simplicity) $\alpha<\lambda=\operatorname{cf}(\lambda) \Rightarrow \lambda^{++} \rightarrow\left(\lambda^{+}+\alpha\right)_{2}^{2}$ (on the problem see [34]), and by Baumgartner, Hajnal and Todorčević [13] for $k<\omega$,

$$
\alpha<\lambda=\operatorname{cf}(\lambda) \Rightarrow \lambda^{++} \rightarrow\left(\lambda^{+}+\alpha\right)_{k}^{2},
$$

the following remains open:

Question 8.6. (GCH) When $\lambda^{++} \rightarrow\left(\lambda^{+}+\alpha\right)_{\aleph_{0}}^{2}$, where $2 \leq \alpha<\lambda$ ? (The GCH assumption is for simplicity only.)

By [165] we know that if $\lambda=\lambda^{<\lambda}$ (e.g., $\lambda=\aleph_{1}=2^{\aleph_{0}}$ ) then possibly $2^{\lambda}$ is very large (in particular $>\lambda^{+\omega}$ ), but $2^{\lambda} \nrightarrow(\lambda \times \omega)_{2}^{2}$. However, $\lambda^{+2 k} \rightarrow$ $(\lambda \times n)_{k}^{2}$ (by [165, bottom of p. 288]), so

QUestion 8.7. For $\lambda=\lambda^{<\lambda}, \lambda>\aleph_{0}$, and $k<\omega$ and $n<\omega$, what is the minimal $m$ such that $\lambda^{+m} \rightarrow(\lambda \times n)_{k}^{2}$ ? (Baumgartner, Hajnal and 
Todorčević [13, p. 2, end of $\S 0]$ prefer to ask whether $\lambda^{++} \rightarrow(\lambda+\omega)_{3}^{2}$ for $\lambda=\aleph_{1}$, so $\lambda=\lambda^{<\lambda}$ means $\mathrm{CH}$ as they choose another extreme case of the unknown.)

Now, for me a try at consistency of negative answers for 8.6 calls for using historic forcing (see Shelah and Stanley [183], Rosłanowski and Shelah [86, $\S 3]$; it is explained below by considering an example). On the other hand, large cardinals may make some positive results easier.

QUESTION 8.8. Assume that $\lambda>\kappa>|\zeta|+\sigma$, and $\kappa$ is a compact cardinal, and $\lambda=\lambda^{<\lambda}$. Does it follow that $\lambda^{+} \rightarrow(\lambda+\zeta)_{\sigma}^{2}$ ?

I tend to think the answer is yes. If so, then we cannot expect, when $\lambda=\lambda^{<\lambda}$, that a $\lambda$-complete $\lambda^{+}$-c.c. forcing notion will add a counterexample $c$ to $\lambda^{+} \rightarrow\left(\lambda+\alpha^{*}\right)_{\aleph_{0}}^{2}$. Let for simplicity $\alpha^{*}=2$. Also we should expect that for such $c$, for every $u \in\left[\lambda^{+}\right]^{<\lambda}$ we can find $f_{n}: u \times u \rightarrow \omega$ for $n<\omega$ such that $f_{n}(\alpha, \alpha)=n$, and for no distinct $\alpha, \beta, \gamma \in u$ do we have:

$$
c(\{\alpha, \beta\})=n=f_{n}(\alpha, \beta) \quad \text { or } \quad f_{n}(\alpha, \beta)=f_{n}(\alpha, \gamma)=c(\{\beta, \gamma\}) .
$$

The point of historic forcing is that we know what kind of object our forcing notion has to add. In our case, we assume $\lambda=\lambda^{<\lambda}$ and a condition $p$ has to give $u^{p} \in\left[\lambda^{+}\right]^{<\lambda}$ and $c^{p}:\left[u^{p}\right]^{2} \rightarrow \omega$ and by the above considerations also $f_{n}: u^{p} \times u^{p} \rightarrow \omega$ (for $n<\omega$ ), and for having some leeway we let $f_{n}: u^{p} \times u^{p} \rightarrow[\omega]^{\aleph_{0}}$ such that $n \in f_{n}(\alpha, \alpha)$, and we demand

(*) for no distinct $\alpha, \beta \in u^{p}$ and $m$ do we have

$$
m=c(\{\alpha, \beta\}) \in f_{n}(\alpha, \beta) \cap f_{n}(\alpha, \alpha),
$$

(**) for no distinct $\alpha, \beta, \gamma$ from $u^{p}$ do we have

$$
c(\{\beta, \gamma\}) \in f_{n}(\alpha, \beta) \cap f_{n}(\alpha, \gamma) .
$$

Moreover, we choose $\left\langle A_{\eta}: \eta \in{ }^{\omega>} \omega\right\rangle, A_{\eta} \in[\omega]^{\aleph_{0}}$, where $\left.\left\langle A_{\eta}\langle l\rangle\right\rangle: l<\omega\right\rangle$ are pairwise disjoint subsets of $A_{\eta}$, and demand $f_{n}(\alpha, \beta) \in\left\{A_{\eta}: \eta \in \omega>\omega\right\}$.

Considering that our forcing will be strategically $(<\lambda)$-complete (as $\lambda$ complete seems too much both if the answer to 8.8 is yes and because of the properties of historic forcing in general), in order that there is no $A \subseteq \lambda^{+}$ with $\operatorname{otp}(A)=\lambda+\alpha^{*}$ such that $\underset{\sim}{c} \uparrow[A]^{2}$ is constant, we have a set $\mathcal{T}$ of cardinality $<\lambda$ such that (for simplicity $\lambda=\mu^{+}$, maybe also $\mu$ regular):

(a) each $x \in \mathcal{T}^{p}$ has the form $x=(a, \delta, b)=\left(a^{x}, \delta^{x}, b^{x}\right)$, where

(i) $\delta<\lambda^{+}, \operatorname{cf}(\delta)=\lambda$,

(ii) $a \subseteq u^{p} \backslash \delta, \operatorname{otp}(a)=\zeta$,

(iii) $b \subseteq u^{p} \cap \delta, \operatorname{otp}(b)=\mu$,

(b) if $\left(a^{\prime}, \delta^{\prime}, b^{\prime}\right),\left(a^{\prime \prime}, \delta^{\prime \prime}, b^{\prime \prime}\right) \in \mathcal{T}^{p}$ then $\left|b^{\prime} \cap b^{\prime \prime}\right|<\mu$ (probably not necessary), 
(c) $c^{p} \uparrow[a \cup b]^{2}$ is constantly $n(x)$,

(d) for no $\alpha \in u^{p} \cap \delta \backslash \sup (b)$ do we have

$$
(\forall \beta \in a \cup b)\left[c^{p}(\{\alpha, \beta\})=n(x)\right] .
$$

A $p$ as above will be a precondition. Now the "history" enters and the proof should be clear just as the definition of the set of the conditions should roll itself.

So atomic conditions will have $u^{p}$ a singleton, each condition will have a history telling how it was created: each step in the history corresponds to one of the reasons for creating a condition in the proof. Naturally, a major reason is the proof of the $\lambda^{+}$-c.c. by the $\Delta$-system lemma. So assume for $l=$ 1,2 that $p_{l} \in \mathbb{P}, \alpha_{1}<\alpha_{2}, u^{p_{1}} \cap \alpha_{1}=u^{p_{2}} \cap \alpha_{2}, u^{p_{1}} \subseteq \alpha_{2}, \operatorname{otp}\left(u^{p_{1}}\right)=\operatorname{otp}\left(u^{p_{2}}\right)$ and the order preserving mapping $\mathrm{OP}_{u^{p_{2}}, u^{p_{1}}}$ from $u^{p_{1}}$ onto $u^{p_{2}}$ maps $p_{1}$ to $p_{2}$. We have to amalgamate $p_{1}$ and $p_{2}$ getting $q$, so we have to determine $f_{n}^{q}$ on $\left(u^{p_{1}} \backslash u^{p_{2}}\right) \times\left(u^{p_{2}} \backslash u^{p_{1}}\right)$, and $c^{q}(\{\alpha, \beta\})$ for $\alpha \in u^{p_{1}} \backslash u^{p_{2}}, \beta \in u^{p_{2}} \backslash u^{p_{1}}$.

But our vision is that there is one line of history, so should the history of $q$ continue the history of $p_{1}$ with $p_{2}$ joining or should the history of $q$ continue the history of $p_{2}$ with $p_{1}$ joining? Both are O.K., but we get two distinct conditions $q^{\prime}, q^{\prime \prime}$ which, however, are equivalent; i.e. $q^{\prime} \sim q^{\prime \prime}:=q^{\prime} \leq q^{\prime \prime} \leq q^{\prime}$.

Generally going back and changing the history as above we get an equivalent condition if this is done finitely many times (this also explains why we get strategical $(<\lambda)$-completeness and not $\lambda$-completeness). That is, we define $p \leq_{\text {pr }} q$ iff $p$ appears in the history of $q, p \sim q$ if $p$ is obtained from $q$ by finitely many changes as above in the history, and lastly $p \leq q$ iff $\left(\exists p^{\prime}\right)\left(p^{\prime} \sim p \& p^{\prime} \leq_{\text {pr }} q\right)$.

What is left? "Only" carrying out the amalgamation (using and guaranteeing the conditions, and probably changing them so that they fit).

QUESTION 8.9. What are the best cardinals needed for the canonization theorems in [140]?

An old well-known problem is

QUESTION 8.10. Is $\aleph_{1} \rightarrow\left[\aleph_{1} ; \aleph_{1}\right]_{2}^{2}$ consistent? (And variants, connected to the $L$-space problem; this seems related to 5.1.)

Another problem of Erdös is (the answer is consistently yes, see [155], even colouring also no edges, but provability in ZFC is not clear):

QUESTION 8.11. Is there a graph $G$ with no $K_{4}$ (complete graph on 4 vertices) such that $G \rightarrow\left(K_{3}\right)_{\aleph_{0}}^{2}$, that is, for any colouring of the edges by $\aleph_{0}$ colours there is a monochromatic triangle?

We can ask it for $K_{k}, K_{k+1}$ instead of $k=3$ and colouring $r$-tuples instead of pairs; the answer still is consistently yes (see [155]), so the problem is in ZFC. See [114, Ch. III, §1] on a connection to model theory. 
A very nice theorem of Hajnal [53] says that, e.g., for any finite graph $G$ and $\kappa$, for some graph $H, H \rightarrow(G)_{\kappa}^{2}$, but leaves as a mystery:

Question 8.12. Let $G$ be a countable graph. Is there a graph $H$ such that

$$
H \rightarrow(G)_{\aleph_{0}}^{2} ?
$$

Starting from a problem of Erdős and Hajnal [33], I have been very interested in consistency results, e.g., of the form $\lambda \rightarrow[\mu]_{3}^{2}, \kappa<\mu<\lambda \leq 2^{\kappa}$ (see [131]). Usually those are really canonization theorems: for a fixed natural colouring, any other is, on a large set, computable from it. Those results help sometimes in consistency results (just as, e.g., the Erdős-Rado theorem helps in ZFC results). Still it seems to me worthwhile to know

Question 8.13. (1) Can we put together the results of, e.g., [131], Shelah and Stanley [182] and [160]? Assume that $\kappa=\kappa^{<\kappa}<\lambda$ where $\lambda$ is, e.g., strongly inaccessible large enough. Can we find a $(<\kappa)$-complete, $\kappa^{+}$-c.c. forcing notion $\mathbb{P}$ such that in $\mathbf{V}^{\mathbb{P}}$ :

(a) for $\sigma<\kappa$ and $\mu<\lambda$ we can find $\mu^{\prime}, \lambda^{\prime}$ such that $\mu<\mu^{\prime}<\lambda^{\prime}<\lambda$ and $\lambda^{\prime} \rightarrow\left[\mu^{\prime}\right]_{\sigma, 2}^{2}$;

(b) if $\kappa$ is a measurable indestructibly by adding many Cohens, then also the parallel results for colouring $n$-tuples (see [160]);

(c) if $\kappa=\aleph_{0}$, we also have results on colouring $n$-tuples simultaneously for all $n$ ?

(2) Add the hopeful consistency answer for 8.14, 8.15.

Probably easier are, e.g.:

QUESTION 8.14. Is it consistent that for some $n, 2^{\aleph_{0}}=\aleph_{n}=\lambda \rightarrow\left[\aleph_{1}\right]_{3}^{2}$ ? (The exact $n$ is less exciting for me, the main division line seems to me $\aleph_{\omega}$, of course best to know the exact $\lambda$.)

QUeSTION 8.15. Is it consistent that $2^{\aleph_{0}}>\lambda \rightarrow\left[\aleph_{1}\right]_{3}^{2} ?$

On finite combinatorics. Spencer, Szemeredi and Alon told me that finding $\lim \left(\log \left(r_{2}^{2}(n) / n\right)\right)$ is a major problem (see Definition 8.16 below), but the difference between the known lower and upper bounds $(1 / 2$ and 2$)$ seems to me negligible.

Definition 8.16. $r_{k}^{m}(n)$ is the minimal $r$ such that $r \rightarrow(n)_{k}^{m}$.

Erdős and Hajnal ask, and I find more convincing, the following. 
Question 8.17. What is the order of magnitude of $r_{2}^{3}(n)$ ?

We expect it should be $2^{2^{n}}$, or e.g. $2^{2^{\left(n^{\varepsilon}\right)}}$ for some $\varepsilon>0$. But we cannot rule out its being $2^{n}$ or e.g. $2^{n^{1 / \varepsilon}}$ for some $\varepsilon>0$. Here the difference is large.

Note that for four colours the problem (what is $r_{4}^{3}(n)$ ) is settled; but I think the true question is:

Question 8.18. Determine (order of magnitude is OK) $f_{k}(n, r), f_{k}^{+}(n, c)$ where:

(a) $f_{3}(n, c)$ is the minimal $m$ such that for every $\mathbf{d}:[m]^{3} \rightarrow\{0, \ldots, c-1\}$, there are $A \in[m]^{n}$ and a strictly increasing function $h: A \rightarrow\left\{0, \ldots, 2^{n}-1\right\}$ such that for $l_{0}<l_{1}<l_{2}$ in $A$, the value of $\mathbf{d}\left(\left\{l_{0}, l_{1}, l_{2}\right\}\right)$ is determined by the quantifier-free type $\left\langle h\left(l_{0}\right), h\left(l_{1}\right), h\left(l_{2}\right)\right\rangle$ in $B_{n}^{3}$, where $B_{n}^{3}$ has the universe $\left\{0, \ldots, 2^{n}-1\right\}$ and two relations: (viewed as ${ }^{n} 2$ ) the lexicographic order and

$$
\left\{\left(\eta_{0}, \eta_{1}, \eta_{2}\right): \eta_{0}<_{\text {lex }} \eta_{1}<_{\text {lex }} \eta_{2} \text { and } \lg \left(\eta_{0} \cap \eta_{1}\right)<\lg \left(\eta_{1} \cap \eta_{2}\right)\right\} \text {. }
$$

(b) $f_{3}^{+}(n, c)$ is defined similarly but for every pregiven $\operatorname{Rang}(h)$ we can find such $h$.

(c) $B_{n}^{k}$ is defined below by induction on $k$, and then $f_{k}(n, c), f_{k}^{+}(n, c)$ are defined analogously to $f_{3}, f_{3}^{+}$.

(d) Define the canonization number $g_{k}(n)$, which is the first $m$ such that: if $d:[n]^{k} \rightarrow C$, with no restriction on the cardinality of $C$, then we can find $A, h$ as above, and a quantifier free formula $\phi$ in the vocabulary of $B_{n}^{k}$ such that for any $u_{1}, u_{2} \in[A]^{k}$ we have

$$
d\left(u_{1}\right)=d\left(u_{2}\right) \quad \text { iff } \quad \phi\left(\ldots, h\left(l_{1}\right), \ldots ; \ldots, h\left(l_{2}\right), \ldots\right)_{\substack{l_{1} \in u_{1} \\ l_{2} \in u_{2}}} \text { is satisfied in } B_{n}^{k} .
$$

Define $g_{n}^{+}(k)$ similarly.

The explicit way to describe $B_{n}^{k}$, by induction on $k$, is: it has a linear order $<_{k} ; B_{n}^{2}$ is the structure $(n,<) ; B_{n}^{k+1}$ has universe $B_{n}^{k}\{0,1\}$ and the relation: $\eta<_{k+1} \nu$ if and only if for some $y=y(\eta, \nu) \in B_{n}^{k}$ we have $\eta(y)=0$, $\nu(y)=1$ and

$$
\eta\left\lceil\left\{x \in B_{n}^{k}: x<_{k} y\right\}=\nu \uparrow\left\{x \in B_{n}^{k}: x<_{k} y\right\} .\right.
$$

For an $m$-place relation $R^{B_{n}^{k}}$ of $B_{n}^{k}, R^{B_{n}^{k+1}}$ is a $2 m$-place relation on $B_{n}^{k+1}$, namely

$$
\begin{aligned}
\left\{\left\langle\eta_{0}, \ldots, \eta_{2 m-1}\right\rangle:\right. & \eta_{l} \in B_{n}^{k+1}, \eta_{l}<_{k+1} \eta_{l+1} \text { and } \\
& \left.\left\langle y\left(\eta_{0}, \eta_{1}\right), y\left(\eta_{2}, \eta_{3}\right), \ldots\right\rangle \in R^{B_{n}^{k}}\right\} .
\end{aligned}
$$

Now it is not clear how fast the number in 8.18 grows, e.g., we cannot exclude $2^{2^{n+c+k}}$. The main question is whether it grows like $h(k)$-iterated 
exponentiation in $n$ (say $c$ fixed) with $h$ going to infinity, or with $h$ constant. Of course, enriching somewhat the structure is not a great loss to me.

Question 8.19. Let $f^{*}(n, c)$ be the first $m$ such that if $\left\langle A_{l}: l<m\right\rangle$ are pairwise disjoint, $\left|A_{l}\right|=m$ for $l<n$ and

$$
F:\left\{w \subseteq \bigcup_{l} A_{l}:\left|w \cap A_{l}\right| \in\{1,2\},(\exists ! l)(|w|=1)\right\} \rightarrow C,
$$

where $|C|=c$ then for some $x_{l} \neq y_{l}$ from $A_{l}$ for $l<n$ we have

$$
l^{*}<n \Rightarrow F\left(\left\{x_{l}, y_{l}: l \neq l^{*}\right\} \cup\left\{x_{l^{*}}\right\}\right)=F\left(\left\{x_{l}, y_{l}: l \neq l^{*}\right\} \cup\left\{y_{l^{*}}\right\}\right) \text {. }
$$

Again the main question for me is: Does $f^{*}(n, c)$ grow as a fixed iterated exponentiation? (This is connected to the van der Waerden theorem, see [154]).

I say more at the end of [122]. On Ramsey theory see Graham, Rothschild and Spencer [49].

Definition 8.20. (1) For a group $G$ and a subset $A$ of $G$, and a group $H$ let $H \rightarrow(G)_{\sigma}^{A}$ mean: if $\mathbf{d}$ is a function with domain $H$ and range of cardinality $\leq \sigma$, then for some embedding $h$ of $G$ into $H$ the function $\mathbf{d}$ restricted to $h(A)$ is constant.

(2) For a group $G$ and a subset $A$ of $G$, and a group $H$ let $H \rightarrow[G]_{\sigma, \tau}^{A}$ mean: if $\mathbf{d}$ is a function with domain $H$ and range of cardinality $\leq \sigma$, then for some embedding $h$ of $G$ into $H$ the range of the function $\mathbf{d}$ restricted to $h(A)$ has cardinality $<\tau$.

If we omit $\tau$, we mean just that the range is not equal to $\sigma$.

(3) For a group $G$ and an equivalence relation $E$ on $G$, and a group $H$ let $H \rightarrow(G)_{\sigma}^{E}$ mean: if $\mathbf{d}$ is a function with domain $H$ and range of cardinality $\leq \sigma$, then for some embedding $h$ of $G$ into $H$, for any $x, y \in G$ which are $E$-equivalent we have $\mathbf{d}(h(x))=\mathbf{d}(h(y))$.

(4) In part (1) (or (2), or (3)) we can replace $A$ (or the domain of $E$ ) by the family of subgroups of $G$ isomorphic to a fixed group $K$, and then $\mathbf{d}$ is a function with domain being the set of subgroups of $G$ isomorphic to $K$.

(5) Like part (4), but we replace "subgroups isomorphic to $K$ " by "embedding of $K$ ", and then replace " $\rightarrow$ " by " $\rightarrow$ " ".

Discussion 8.21. There is a connection between the last two definitions: the first implies a special case of the second one, when we restrict ourselves to permutation groups of some finite set, and $A$ is the set of conjugates of the permutation just interchanging two elements.

Problem 8.22. (1) Investigate the arrows from Definition 8.20.

(2) In particular, consider the case when $A$ is a set of pairwise conjugate members of $G$ each of order two. 


\section{Except forcing}

Problem 9.1. Are there methods to prove independence except forcing?

As mathematicians do not report their failures, not much is said in the literature. I do not mean results that simply follow from the consistency strength, e.g., by [146] in some forcing extensions of $\mathbf{L}$ by a forcing notion not collapsing cardinals (in fact satisfying the c.c.c.), $\mathrm{PB} \equiv$ "every projective set of reals has the Baire property", whereas the consistency strength of PM $\equiv$ " every projective set of reals is Lebesgue measurable" is larger. Hence we have the consistency with $\mathrm{ZFC}$ of $\mathrm{PB}+\neg \mathrm{PM}$, but we cannot use it to prove the consistency of $\mathrm{ZFC}+\neg \mathrm{PB}+\mathrm{PM}$ (on the other hand see hopefully [125]). The point is that the issue of the consistency strength is elsewhere, so it gives such results as a byproduct, and we do not have strong control, i.e., we would like to have:

DESIRE 9.2. A method to get independence of statements starting with the problem and then having a natural direction.

A major issue of set theory is:

DREAM 9.3. Find a parallel of forcing for $\mathrm{ZFC}+\mathbf{V}=\mathbf{L}$ (or even for ZFC with no statements on $\mathbf{L}$ ) and even more so:

Dream 9.4. Find a parallel of forcing for PA (or even ZFC with statements on number theory).

A possible direction is trying to construct non-well-founded models (note that well-foundedness is inherent in forcing as it preserves the ordinals). It would be interesting for set theory:

Speculation 9.5. In ZFC + "there is a supercompact cardinal" we can prove that the theory

$$
\mathrm{ZFC}+\text { "there is a compact cardinal" }
$$

has a model, but not a well-founded model.

This would explain well why it remains a mystery if supercompact and compact have the same consistency strength (Magidor proved the first compact may be the first measurable or first supercompact; but having no inner model, the usual method does not work now).

But back to 9.3, a traditional way to attack such problems is to choose a good test question; for independence from $\mathrm{ZFC}, \mathrm{CH}$ has served excellently. Unfortunately, for independence from $\mathrm{ZFC}+\mathbf{V}=\mathbf{L}$, there is no obvious candidate. Now, trying to shoot the enemy without seeing him may end, as many times, in first shooting the arrow and then marking the target, an old practice. 
It is not totally unreasonable that forcing might be unique in some sense. I mean the syntactical statements. Note that the theories should have the same ordinals in some sense, and we should exclude forcing with a proper class, so we should in some sense restrict ourselves to bounded sentences, i.e.,

$$
(\exists \chi)((\mathcal{H}(\chi), \in) \models \psi) .
$$

Definition 9.6. For bounded sentences $\psi_{1}, \psi_{2}$ let $\psi_{1} \leq_{\mathrm{cs}} \psi_{2}$ mean that for every finite subset $\Phi$ of $\mathrm{ZFC}+\psi_{1}$, in $\mathrm{ZFC}+\psi_{2}$, we can prove it has a well-founded model.

Still we have here a problem: by playing with statements on the existence of models we can find "erratic" behaviour which it is widely felt does not occur in "NMM (= non-metamathematical) statements". (I do not call them mathematical, as the sentences like $\mathrm{CON}(\mathrm{ZFC}+$ there exists an inaccessible cardinal) are excellent mathematical statements in my eyes). See [57] and [191] on finding NMM statements closely related to such statements.

This appears in several directions.

Problem 9.7. Can we find a large family $\Phi_{l}$ of bounded sentences in set theory which formalize "being NMM" at least to some extent, (and so at least) is reasonably wide and natural, for which we answer at least some of the following:

$(A)_{0} \quad$ for $\Phi_{0}, \mathrm{ZFC}+\mathbf{V}=\mathbf{L}$ is complete, i.e.,

$$
\psi \in \Phi_{0} \quad \Rightarrow \quad[\mathrm{ZFC}+\mathbf{V}=\mathbf{L} \vdash \psi \text { or } \mathrm{ZFC}+\mathbf{V}=\mathbf{L} \vdash \neg \psi] .
$$

[This is the other side of the lack of good candidates, for in other words, the successes in solving the Suslin hypothesis, the Kurepa hypothesis, $n$-cardinal transfer theorems in $\mathbf{L}$ deprives us of good candidates.] As large cardinals may enter, we may consider:

$(A)_{1} \quad$ For $\psi \in \Phi_{1}, \mathrm{ZFC}+$ "there exists a supercompact cardinal" decides $\psi^{\mathbf{L}}$.

$(A)_{2} \quad$ For all $\psi_{1}, \psi_{2} \in \Phi_{2}$, the consistency strengths of $\mathrm{ZFC}+\psi_{1}$ and $\mathrm{ZFC}+\psi_{2}$ are comparable.

$(A)_{3} \quad$ If $\mathrm{ZFC}+\psi$ is consistent, $\psi \in \Phi_{3}$, then we can get it by forcing.

We may consider weakenings. Of course, judging the success may be disputable; in fact, I am sure that 9.3, 9.4 will eventually be solved, whereas 9.7 probably will remain with answers like "completeness for reasonable logics in $\mathbf{L}$ " (which is still very interesting).

There are works on independence for fragments of PA (with bounded induction, without $(\forall x)\left(2^{x}\right.$ exists) (possibly with $(\forall x)\left(x^{[\log (x)]}\right.$ exists)), they speak about pigeonhole principles when we add a new set as a predicate (see 
Ajtai [5]); this seems to me parallel to the Fraenkel-Mostowski method. I have thought about it lately because of [124].

Concerning 9.3, let us consider the following thought.

In $\mathrm{ZFC}+\mathbf{V}=\mathbf{L}$, every element has the form

$$
\tau_{\varphi(x, \bar{y})}(\alpha, \bar{\beta})=\left\{x \in \mathbf{L}_{\alpha}:\left(\mathbf{L}_{\alpha}, E\right) \models \varphi(x, \bar{\beta})\right\},
$$

where $\bar{\beta}=\left\langle\beta_{0}, \ldots, \beta_{n-1}\right\rangle, \beta_{l}<\alpha$ (without loss of generality, $\beta_{0}<\beta_{1}<\ldots$ ) and $\varphi$ is first order. So let $\mathbf{I}$ be a linear order (which later can be nonstandard) and let $\bar{\Phi}=\left\langle\Phi_{l}: l<n^{*}\right\rangle$ be an increasing sequence of sets of first order formulas (in set theory vocabulary $\{\in\}$ ). We may consider trying to define $\left\langle M_{\mathbf{I}, \Phi_{l}}: l<n^{*}\right\rangle$ with $M_{\mathbf{I}, \Phi_{l}}=\left\{\tau_{\varphi}(a, \bar{b}): a \in \mathbf{I}, b_{l}<_{\mathbf{I}} a, \varphi \in \Phi_{l}\right\}$ as in $[149, \S 1]$.

\section{Recent advances/comments}

Section 1. During the winter of 1999, Gitik told me that he can start with

$\mathbf{V} \models " \kappa_{n}$ hypermeasurable of order $\lambda_{n}$,

$\lambda_{n}$ first (strongly) inaccessible $>\kappa_{n}, \lambda_{n}<\kappa_{n+1}, \lambda>\kappa=\sum_{n} \kappa_{n} "$,

and find a forcing notion $\mathbb{P}$, not adding bounded subsets of $\kappa=\sum_{n<\omega} \kappa_{n}$, satisfying the $\kappa^{++}$-c.c., and making $2^{\kappa} \geq \lambda$. I have conjectured that combining this proof with earlier proofs, you can demand that forcing with $\mathbb{P}$ makes $\lambda_{n}$ be the $n$th inaccessible cardinal, $\kappa=\sum_{n} \lambda_{n}$, GCH holds below $\kappa$ and $2^{\kappa} \geq \lambda$, so $\operatorname{pp}(\kappa) \geq \lambda$. Gitik has confirmed this conjecture with $\lambda_{n}$ the $n$th Mahlo cardinal. This proves that though 1.20 is open, other theorems which hold for $\lambda$ of cofinality $\aleph_{1}$ cannot be generalized to cofinality $\aleph_{0}$.

On what the pcf theory tells you what you cannot do toward proving the consistency of the negation of the weak hypothesis 1.12(A) see Gitik and Shelah [43].

Section 2. Concerning 2.2, 3.7, i.e., 2.13 and more generally on making the continuum large, see related work in progress [111].

Section 3. Question 3.2 seems to be solved (see [101]), but certainly not along the lines described here. We show $\operatorname{CON}(\mathfrak{a}>\mathfrak{d})$ in the following way: we make the continuum large, and use ultrapower of the forcing notion with model-theoretic point of view. This works also for $\mathfrak{u}$. We may wonder: Does the method have other applications? However, we do not know

Question 10.1. (1) Is ZFC $+2^{\aleph_{0}}=\aleph_{2}$ consistent with $\mathfrak{a}>\mathfrak{d}$ ?

(2) What is the consistency strength of ZFC $+\mathfrak{a}>\mathfrak{u}$ ? (For $\mathfrak{a}>\mathfrak{d}, \mathrm{ZFC}$ suffices.) 
Section 4. Some examples of forcing notions as required in Problem 4.24 are given in Rosłanowski and Shelah [89, §1].

Section 5. Regarding $(\otimes)$ (formulated after 5.4), let us state explicitly:

THEOREM 10.2. If $\kappa$ is a regular cardinal then one of the following occurs:

(a) there is a subset $S$ of $\kappa^{+}$with a square on it (see $(\otimes)($ a) after 5.4$)$ and such that stationarily many $\delta \in S$ have cofinality $\kappa$,

(b) there is a subset $S$ of $\kappa^{++}$with square on it and such that stationarily many $\delta \in S$ have cofinality $\kappa$ and $S \cap S_{\kappa}^{\kappa^{++}}$does not reflect in any ordinal of cofinality $\kappa^{+}$.

I think that 10.2 is nice and useful, e.g., for constructing objects in $\aleph_{n}$ for infinitely many $n$. In fact, I mention it as lately Eklof told me he has built sufficiently separable Abelian groups giving negative answers to Kaplansky's test problems in every $\aleph_{n}$ by induction on $n$, using, for $n<\omega$, the statement $I\left[\aleph_{n+2}\right] \mid S_{\aleph_{n+1}}^{\aleph_{n+2}}$ is not trivial (that is, there is a stationary subset $S$ of $S_{\aleph_{n+1}}^{\aleph_{n+2}}$ which belongs to $I\left[\aleph_{n+2}\right]$ ). I have advised him to use 10.2 (at the price of advancing from $n$ to $n+1$ or to $n+2)$.

Pro of (of Theorem 10.2). We prove slightly more. Let $\lambda$ be regular, $\theta=\operatorname{cf}(\theta)<\lambda$ (in the theorem as stated $\left.\lambda=\kappa^{+}, \theta=\kappa\right)$. By [159, §4], there are $S \subseteq \lambda^{+}$and a square $\left\langle C_{\delta}: \delta \in S\right\rangle$ (as defined in $\otimes($ a) mentioned above), such that:

- $\delta \in S \Rightarrow \operatorname{otp}\left(C_{\delta}\right)<\lambda$, and

- $S_{1}:=\{\delta \in S: \operatorname{cf}(\delta)=\theta\}$ is a stationary subset of $\lambda^{+}$.

Let $\gamma(*)<\lambda$ be the minimal $\gamma$ such that the set

$$
S_{\gamma}^{1}:=\left\{\delta \in S_{1}: \operatorname{otp}\left(C_{\delta}\right)=\gamma\right\}
$$

is stationary (so necessarily $\operatorname{cf}(\gamma(*))=\theta$ ). Let $e$ be a closed unbounded subset of $\gamma(*)$ of order type $\theta$ such that

$$
\varepsilon \in \operatorname{nacc}(e) \Rightarrow \varepsilon \text { is a successor ordinal. }
$$

Let

$$
S_{2}:=\left\{\delta \in S: \operatorname{otp}\left(C_{\delta}\right) \in e \cup\{\gamma(*)\}\right\},
$$

and for $\alpha \in S$ let $C_{\alpha}^{2}:=C_{\alpha} \cap S_{2}$. Clearly $S_{\gamma(*)}^{1} \subseteq S_{2}$, and $\alpha \in S_{2} \Rightarrow \operatorname{otp}\left(C_{\alpha}^{2}\right)$ $\leq \theta$.

CASE 1: For some $\delta^{*}<\lambda^{+}$of cofinality $\lambda$, the intersection $S_{\gamma(*)}^{1} \cap \delta^{*}$ is a stationary subset of $\delta^{*}$. Let $\left\langle\alpha_{\varepsilon}: \varepsilon<\lambda\right\rangle$ be an increasing continuous sequence of ordinals $<\delta^{*}$ with limit $\delta^{*}$ such that for any non-limit $\varepsilon<\lambda$, the ordinal $\alpha_{\varepsilon}$ is non-limit. Let

$$
S^{*}:=\left\{\varepsilon<\lambda: \alpha_{\varepsilon} \in S_{2}\right\}
$$


and for $\varepsilon \in S^{*}$ let $C_{\varepsilon}^{*}:=\left\{\zeta<\varepsilon: \alpha_{\zeta} \in C_{\alpha_{\varepsilon}}^{2}\right\}$. Now clearly $S^{*}$ is a stationary subset of $\lambda$, and

$$
S_{1}^{*}:=\left\{\varepsilon \in S^{*}: \operatorname{cf}(\varepsilon)=\theta\right\}
$$

is a stationary subset of $\lambda$, and is equal to $\left\{\varepsilon<\lambda: \alpha_{\varepsilon} \in S_{\gamma(*)}^{1}\right\}$.

If $\theta=\aleph_{0}$, by [159, 44 , we are done (without using the above).

So we can assume that $\theta>\aleph_{0}$; hence $\varepsilon \in S_{1}^{*} \Rightarrow \varepsilon=\sup \left(C_{\varepsilon}^{*}\right)$. Also, $\operatorname{otp}\left(C_{\varepsilon}^{*}\right) \leq \theta$ and $C_{\varepsilon}^{*}$ is a closed subset of $\varepsilon$ for $\varepsilon \in S^{*}$, and

$$
\zeta \in C_{\varepsilon}^{*} \& \varepsilon \in S^{*} \Rightarrow C_{\zeta}^{*}=C_{\varepsilon}^{*} \cap \zeta \& \zeta \in S^{*} .
$$

So $\left\langle C_{\varepsilon}^{*}: \varepsilon \in S^{*}\right\rangle$ is as required in clause (a) of the theorem, except the requirement

(*) if $\varepsilon$ is a limit ordinal in $S^{*}$, then $C_{\varepsilon}^{*}$ is unbounded in $\varepsilon$.

But this is a minor point - we can replace such $\varepsilon$ 's by their successors. That is, let $S^{* *}=\left\{\varepsilon\right.$ : for some $\zeta \in S^{*}$ we have $\left(\zeta=\sup \left(C_{\zeta}^{*}\right) \Rightarrow \varepsilon=\zeta\right)$ and $\left(\zeta>\sup \left(C_{\zeta}^{*}\right) \Rightarrow \varepsilon=\zeta+1\right)$. If $\varepsilon \in S^{* *}$ and $\zeta \in S^{*}$ are as above, let $C_{\varepsilon}^{* *}=\left\{\alpha\right.$ : for some $\beta \in C_{\zeta}^{*}$ we have $\left(\beta=\sup \left(C_{\beta}^{*}\right) \Rightarrow \alpha=\beta\right)$ and $(\beta>$ $\left.\left.\sup \left(C_{\zeta}^{*}\right) \Rightarrow \alpha=\beta+1\right)\right\}$.

CASE 2: Not case 1. Then clause (b) of our theorem holds as exemplified by $\left\langle C_{\alpha}^{2}: \alpha \in S_{2}\right\rangle$.

Concerning the discussion on 5.12 ("is there an entangled linear order of cardinality $\lambda^{+}$such that $\lambda=\lambda^{\aleph_{0}}$ ?"), we mentioned that if $\mu$ is strong limit singular of uncountable cofinality and $2^{\mu}>\aleph_{\mu^{+}}$, then it holds. Let me justify this:

Proposition 10.3. (1) Assume:

(a) $\mu$ is a singular cardinal of cofinality $\kappa$,

(b) $\lambda=\aleph_{\mu^{+}}$,

(c) $\mu$ is strong limit, $\lambda \leq 2^{\mu}$ or at least

(c) ${ }^{-} \mu=\mu^{<\kappa}$ is a limit cardinal, $\lambda \leq \mu^{\kappa}$.

Then there is an entangled linear order of cardinality $\lambda^{+}$and $\lambda=\lambda^{<\kappa}$.

(2) Assume:

(a) $\kappa=\operatorname{cf}(\kappa)<\mu \leq \operatorname{cf}(\lambda)<\lambda$,

(b) $\lambda \leq \mu^{\kappa}, \mu=\mu^{<\kappa}$, or at least there is a tree with $\kappa$ levels, $\leq \mu$ nodes and at least $\lambda \kappa$-branches,

(c) if $\mathfrak{a}$ is a set of $<\kappa$ regular cardinals from the interval $(\mu, \lambda)$, then $\max \operatorname{pcf}(\mathfrak{a})<\lambda$

(d) if $\theta \in(\mu, \lambda)$ and $\chi$ is the minimal singular cardinal $\chi \in(\theta, \lambda)$ such that $\operatorname{pp}(\chi) \geq \lambda^{+}$, then $\operatorname{cf}(\chi) \leq \kappa$.

Then there is an entangled linear order of cardinality $\lambda^{+}$. 
Proof. (1) First note that

$(*)_{1} \quad$ if $\theta<\lambda$ then $\theta^{<\kappa}<\lambda$.

[Why? By [166, Ch. IX, 4.2, p. 394] as $\mu^{<\kappa}=\mu<\lambda=\aleph_{\mu^{+}}$, or see [164].]

$(*)_{2} \quad \lambda^{<\kappa}=\lambda$.

$\left[\right.$ Why? By $(*)_{1}$ as $\operatorname{cf}(\lambda)>\kappa$.]

If for some $\alpha<\mu^{+}$, for every $\beta \in\left[\alpha, \mu^{+}\right)$we have

$$
\max \operatorname{pcf}\left(\left\{\aleph_{\gamma+1}: \gamma \in[\alpha, \beta)\right\}\right)<\lambda,
$$

then we can get the desired conclusion as in the proof of $[164,4.2$, p. 416, case I]. So assume

$(*)_{3} \quad$ for every $\alpha<\mu^{+}$, for some $\beta \in\left[\alpha, \mu^{+}\right)$we have

$$
\lambda \leq \max \operatorname{pcf}\left(\left\{\aleph_{\gamma+1}: \gamma \in[\alpha, \beta)\right\}\right) .
$$

Note that equality never holds as $\lambda$ is singular.

For every $\alpha<\mu^{+}$which is above $\mu$ let $\beta(\alpha) \in\left(\alpha, \mu^{+}\right)$be the first ordinal $\beta$ such that $\max \operatorname{pcf}\left(\left\{\aleph_{\gamma+1}: \gamma \in[\alpha, \beta)\right\}\right)>\lambda$. So $\aleph_{\beta(\alpha)}$ is a singular cardinal $>\aleph_{\alpha}$ of cofinality $\leq \mu$ such that $\lambda \leq \operatorname{pp}\left(\aleph_{\beta(\alpha)}\right)$. Choose by induction on $i<\mu^{+}$an ordinal $\alpha(i)<\mu^{+}$such that $j<i \Rightarrow \beta(\alpha(j))<\alpha(i)$. Let $\alpha(*)=\sup \{\alpha(i): i<\mu\}$ and let

$$
\mathfrak{a}_{i}=\left\{\theta: \theta \text { is a regular cardinal } \in\left[\aleph_{\alpha(i)}, \aleph_{\alpha(i+1)}\right)\right\}
$$

and

$$
\mathfrak{b}_{i}=\left\{\theta \in \mathfrak{a}_{i}: \theta \in \operatorname{pcf}(\mathfrak{c}) \text { for no set } \mathfrak{c} \subseteq \mathfrak{a}_{i} \cap \theta \text { of cardinality } \leq \mu\right\} .
$$

Now, for every $i<\mu$ and regular $\theta \in\left[\aleph_{\alpha(*)}, \lambda\right)$ there is a subset $\mathfrak{c}_{\theta, i}$ of $\mathfrak{a}_{i}$ of cardinality $\leq \mu$ such that $\theta=\max \operatorname{pcf}\left(\mathfrak{c}_{\theta, i}\right)$. In fact, we can assume $\mathfrak{c}_{\theta, i} \subseteq \mathfrak{b}_{i}$. By [166, Ch. II, 4.11, p. 81], this implies that (on Ens see [174, $\S 1])$ :

$(*)_{4} \quad$ we have $\operatorname{Ens}(\theta, \mu)$ for any regular $\theta \in\left[\aleph_{\alpha(*)}, \aleph_{\mu^{+}}\right)$.

Let $\delta^{*}$ be the first limit ordinal $\delta>\alpha(*)$ such that $\lambda \leq \operatorname{pp}\left(\aleph_{\delta}\right)$. By a previous assumption, $\delta^{*}<\mu^{+}$, hence $\operatorname{cf}\left(\delta^{*}\right)<\mu$, and also $\kappa \leq \operatorname{cf}\left(\delta^{*}\right)$ by $(*)_{1}$. Can $\operatorname{cf}\left(\delta^{*}\right)>\kappa$ ? No, by $[164,3.4$, p. 412].

So necessarily $\operatorname{cf}\left(\delta^{*}\right)=\kappa$ and we can find a set $\mathfrak{a}$ of $\leq \kappa$ regular cardinals in the interval $\left(\aleph_{\alpha(*)}, \aleph_{\delta^{*}}\right)$ such that:

(i) $\max \operatorname{pcf}(\mathfrak{a})=\lambda^{+}$,

(ii) if $\theta \in \mathfrak{a}$ then $\max \operatorname{pcf}(\mathfrak{a} \cap \theta)<\theta$,

(iii) $\mathfrak{a}$ is unbounded in $\aleph_{\delta^{*}}$.

Recall that $\operatorname{Ens}(\theta, \mu)$ for every $\theta \in \mathfrak{a}$, so by the proposition below we are done.

(2) Similarly, using sets of at most $\operatorname{cf}(\lambda)$ regular cardinals in $(\mu, \lambda)$. 


\section{Proposition 10.4. (1) Assume:}

(a) $\mathfrak{a}$ is a set of regular cardinals $>|\mathfrak{a}|$,

(b) for every $\theta \in \mathfrak{a}$ we have $\max \operatorname{pcf}(\mathfrak{a} \cap \theta)<\theta$,

(c) $J$ is an ideal on $\mathfrak{a}$ extending $J_{\mathfrak{a}}^{\mathrm{bd}}$,

(d) $\prod \mathfrak{a} / J$ has true cofinality $\lambda$,

(e) $\operatorname{cf}(\sup (\mathfrak{a}))=\kappa$,

(f) $\left\langle\mu_{i}: i<\kappa\right\rangle$ is a non-decreasing sequence of cardinals such that $\prod_{j<i} \mu_{j} \leq \mu_{i}$,

(g) $\sup (\mathfrak{a}) \leq \prod_{j<\kappa} \mu_{j}$,

(h) $\left\langle i_{\theta}: \theta \in \mathfrak{a}\right\rangle$ is non-decreasing with limit $\kappa$ and $\operatorname{Ens}\left(\theta, \mu_{i_{\theta}}\right)$ for $\theta \in \mathfrak{a}$.

Then there is an entangled linear order of cardinality $\lambda^{+}$.

(2) In part (1), we can omit assumption (f) and do not require $\left\langle i_{\theta}: \theta \in \mathfrak{a}\right\rangle$ to be non-decreasing, but then we demand:

(i) for each $\theta^{*} \in \mathfrak{a},\left\{\theta \in \mathfrak{a}: i_{\theta}=i_{\theta^{*}}\right\} \notin J_{<\lambda}[\mathfrak{a}]$, where $\lambda=$ $\max \operatorname{pcf}(\mathfrak{a})$,

(j) $\left\langle E_{i}: i<\kappa\right\rangle$ is a sequence of equivalence relations on $\lambda$ such that for every finite $w \subseteq \lambda$, for some $i, E_{i}\lceil w$ is the equality.

Proof. Let $\left\langle f_{\alpha}: \alpha<\lambda\right\rangle$ be a sequence of pairwise distinct members of $\prod \mathfrak{a}$ such that for each $\theta \in \mathfrak{a}$ the set $T_{\theta}=\left\{f_{\alpha}\lceil\theta: \alpha<\lambda\}\right.$ has cardinality $<\theta$ - exists by the assumptions (the only place we use $\prod \mathfrak{a} / J$ has true cofinality $\lambda$ ); see [166, II, 3.1].

Let $\langle\theta(\zeta): \zeta<\kappa\rangle$ be a strictly increasing sequence of cardinals $\in \mathfrak{a}$, unbounded in it. Let $\left\langle E_{i, j}: j<\kappa\right\rangle$ be a sequence of equivalence relations on $T_{\theta(i)}, E_{i, j}$ having $\leq \mu_{j}$ classes and $\left[j_{1}<j_{2} \Rightarrow E_{i, j_{2}}\right.$ refines $\left.E_{i, j_{1}}\right]$ and $\bigcap_{j} E_{i, j}$ is the equality. For $\theta \in \mathfrak{a}$ let $\zeta(\theta)=\min \{\zeta: \theta \leq \theta(\zeta)\}$ and let $E_{\theta}$ be the following equivalence relation on $T_{\theta}: f E_{\theta} g$ if and only if $f, g \in T_{\theta}$ and for every $i, j<\zeta$ we have $\left(f\lceil i) E_{i, j}(g\lceil i)\right.$.

Clearly

$(*)_{5} \quad E_{\theta}$ is an equivalence relation with $\leq \mu_{\zeta(\theta)}$ equivalence classes,

$(*)_{6} \quad$ if $\theta<\chi$ are from $\mathfrak{a}$ and $f, g \in T_{\chi}$ and $f E_{\chi} g$ then $\left(f\lceil\theta) E_{\theta}(g \mid \theta)\right.$.

So for each $\theta \in \mathfrak{a}$ and $f \in T_{\theta}$ we can choose a linear order $<_{f}$ on $\theta$ such that

$(*)_{7} \quad$ if $f E_{\theta} g$ then $<_{f}=<_{g}$,

$(*)_{8} \quad$ if $n<\omega$ and $f_{1}, \ldots, f_{n} \in T_{\theta}$ are pairwise non- $E_{\theta}$-equivalent then $\left\langle\left(\theta,<_{m}\right): m=1, \ldots, n\right\rangle$ is an entangled sequence of linear orders.

Now we linearly order $\left\{f_{\alpha}: \alpha<\lambda\right\}$ by:

$(*)_{9} \quad$ if $f_{\alpha} \uparrow \theta=f_{\beta} \uparrow \theta$, call it $g$, and $f_{\alpha}(\theta)<f_{\beta}(\theta)$ then:

$f_{\alpha}<^{*} f_{\beta}$ if and only if $f_{\alpha}(\theta)<_{g} f_{\beta}(\theta)$.

The rest as in [166, Ch. II, Section 4]. 
Section 6. Concerning questions from Monk [84] of the form "can the invariant of the ultraproduct of the Boolean algebras be smaller than the ultraproducts of the invariants of the $B_{i}$ ", Magidor and Shelah [77] deal with consistency results. This is continued in Shelah and Spinas [181]; see more in [121].

Concerning [84, Problems 10, 11] on depth see [112].

About 6.7(2): By [106], the answer is no, i.e., if $B$ is a superatomic Boolean algebra with $>\beth_{4}(\theta)$ elements, then it has an automorphism moving $>\theta$ atoms. This is essentially reduced to problems on $\operatorname{Pr}_{\text {ad }}\left(\lambda, \lambda, \theta, \aleph_{0}\right)$ (see definition below).

Definition 10.5. $\operatorname{Pr}_{\text {ad }}(\mu, \lambda, \theta, \kappa)$ means that there is a family $\mathcal{A}$ of $\mu$ subsets of $\lambda$ each of cardinality $\kappa$, the intersection of any two of cardinality $<\kappa$, such that any subset of $\lambda$ of cardinality $\theta$ contains one of them. (Mild pcf conjectures guarantee existence in many cases.)

Added in proof. Continuing $\S 10$ (on $\S 1$ ) Gitik solved 1.20 proving consistency.

Concerning 2.11(1), it seems [88] proves the consistency.

Concerning 3.9, see recent work of Shelah and Steprāns [185].

\section{References}

[1] U. Abraham, Aronszajn trees on $\aleph_{2}$ and $\aleph_{3}$, Ann. Pure Appl. Logic 24 (1983), $213-230$.

[2] U. Abraham, M. Rubin and S. Shelah, On the consistency of some partition theorems for continuous colorings, and the structure of $\aleph_{1}$-dense real order types, Ann. Pure Appl. Logic 29 (1985), 123-206.

[3] U. Abraham and S. Shelah, Isomorphism types of Aronszajn trees, Israel J. Math. 50 (1985), 75-113.

[4] U. Abraham and S. Todorčević, Partition properties of $\omega_{1}$ compatible with CH, Fund. Math. 152 (1997), 165-181.

[5] M. Ajtai, The complexity of the pigeonhole principle, Combinatorica 14 (1994), $417-433$.

[6] U. Avraham [U. Abraham], K. J. Devlin and S. Shelah, The consistency with CH of some consequences of Martin's axiom plus $2^{\aleph_{0}}>\aleph_{1}$, Israel J. Math. 31 (1978), 19-33.

[7] T. Bartoszyński and H. Judah, Set Theory: On the Structure of the Real Line, A K Peters, Wellesley, MA, 1995.

[8] T. Bartoszyński, A. Rosłanowski and S. Shelah, After all, there are some inequalities which are provable in ZFC, math.LO/9711222 $\left(^{4}\right)$; J. Symbolic Logic 65 (2000), 803-816.

[9] - - - - , Adding one random real, ibid. 61 (1996), 80-90; math.LO/9406229.

$\left({ }^{4}\right)$ References of the form math.XX/ $\cdots$ refer to the xxx.lanl.gov archive. 
[10] J. E. Baumgartner, Decomposition of embedding of trees, Notices Amer. Math. Soc. 17 (1970), 967.

[11] -, All $\aleph_{1}$-dense sets of reals can be isomorphic, Fund. Math. 79 (1973), 101-106.

[12] - Ultrafilters on $\omega$, J. Symbolic Logic 60 (1995), 624-639.

[13] J. Baumgartner, A. Hajnal and S. Todorčević, Extensions of the Erdős-Rado Theorems, in: Finite and Infinite Combinatorics in Set Theory and Logic, Kluwer, 1993, 1-18.

[14] S. Ben David, On Shelah's compactness of cardinals, Israel J. Math. 31 (1978), $34-56$ and 394 .

[15] A. Blass and S. Shelah, There may be simple $P_{\aleph_{1}}$ - and $P_{\aleph_{2}}$-points and the Rudin-Keisler ordering may be downward directed, Ann. Pure Appl. Logic 33 (1987), 213-243.

[16] - - - Ultrafilters with small generating sets, Israel J. Math. 65 (1989), 259-271.

[17] R. Bonnet and D. Monk, Handbook of Boolean Algebras, Vols. 1-3, NorthHolland, 1989.

[18] J. Brendle and S. Shelah, Ultrafilters on $\omega$-their ideals and their cardinal characteristics, Trans. Amer. Math. Soc. 351 (1999), 2643-2674; math.LO/9710217.

[19] T. J. Carlson, Strong measure zero and strongly meager sets, Proc. Amer. Math. Soc. 118 (1993), 577-586.

[20] K. Ciesielski, Set theoretic real analysis, J. Appl. Anal. 3 (1997), 143-190.

[21] K. Ciesielski and S. Shelah, Category analog of sub-measurability problem, ibid., to appear; math.LO/9905147.

[22] J. Cummings, M. Džamonja and S. Shelah, A consistency result on weak reflection, Fund. Math. 148 (1995), 91-100; math.LO/9504221.

[23] J. Cummings and M. Foreman, The tree property, Adv. Math. 133 (1998), $1-32$.

[24] J. Cummings and S. Shelah, A model in which every infinite Boolean algebra has many subalgebras, J. Symbolic Logic 60 (1995), 992-1004; math.LO/9509227.

[25] - - - Cardinal invariants above the continuum, Ann. Pure Appl. Logic 75 (1995), 251-268; math.LO/9509228.

[26] K. J. Devlin and S. Shelah, A weak version of $\diamond$ which follows from $2^{\aleph_{0}}<2^{\aleph_{1}}$, Israel J. Math. 29 (1978), 239-247.

[27] T. Dodd and R. B. Jensen, The covering lemma for K, Ann. Math. Logic 22 (1982), 1-30.

[28] M. Džamonja and S. Shelah, Universal graphs at successors of singular strong limits, preprint.

[29] - - - Weak reflection at successors of singulars, preprint.

$[30]-,-$, On squares, outside guessing of clubs and $I_{<f}[\lambda]$, Fund. Math. 148 (1995), 165-198; math.LO/9510216.

[31] M. Džamonja and S. Shelah, Saturated filters at successors of singulars, weak reflection and yet another weak club principle, Ann. Pure Appl. Logic 79 (1996), 289-316; math.LO/9601219.

[32] P. C. Eklof and A. Mekler, Almost Free Modules; Set Theoretic Methods, NorthHolland Library, 1990.

[33] P. Erdős and A. Hajnal, Unsolved problems in set theory, in: Axiomatic Set Theory, Proc. Sympos. Pure Math., 13, part I, Providence, RI, 1971, Amer. Math. Soc., 17-18.

[34] P. Erdős, A. Hajnal, A. Maté and R. Rado, Combinatorial Set Theory: Partition Relations for Cardinals, Stud. Logic Found. Math. 106, North-Holland, Amsterdam, 1984. 
[35] W. G. Fleissner and S. Shelah, Collectionwise Hausdorff: incompactness at singulars, Topology Appl. 31 (1989), 101-107.

[36] M. Foreman and H. Woodin, The generalized continuum hypothesis can fail everywhere, Ann. Math. 133 (1991), 1-36.

[37] D. Fremlin, Problem list, circulated notes, 1994.

[38] -, Real-valued-measurable cardinals, in: Set Theory of the Reals, Israel Math. Conf. Proc. 6, Bar-Ilan Univ., Ramat Gan, 1993, 151-304.

[39] L. Fuchs, Infinite Abelian Groups, Vols. I, II, Academic Press, New York, 1970, 1973.

[40] S. Garcia-Ferreira and W. Just, Two examples of relatively pseudocompact spaces, Questions Answers Gen. Topology 17 (1999), 35-45.

[41] M. Gitik, All uncountable cardinals can be singular, Israel J. Math. 35 (1980), 61-88.

[42] M. Gitik and S. Shelah, More on real-valued measurable cardinals and forcing with ideals, Israel J. Math., submitted; math.LO/9507208.

[43] -, 一, On some configurations related to Shelah's weak hypothesis, Arch. Math. Logic, to appear; math.LO/9909087.

[44] -, - Forcings with ideals and simple forcing notions, Israel J. Math. 68 (1989), $129-160$.

[45] - - - On certain indestructibility of strong cardinals and a question of Hajnal, Arch. Math. Logic 28 (1989), 35-42.

[46] - - - More on simple forcing notions and forcings with ideals, Ann. Pure Appl. Logic 59 (1993), 219-238.

[47] -, -, Less saturated ideals, Proc. Amer. Math. Soc. 125 (1997), 1523-1530; math.LO/9503203.

[48] M. Goldstern and S. Shelah. Many simple cardinal invariants, Arch. Math. Logic 32 (1993), 203-221; math.LO/9205208.

[49] R. Graham, B. L. Rothschild and J. Spencer, Ramsey Theory, Wiley, New York, 1980.

[50] J. Gregory, Higher Souslin trees and the generalized continuum hypothesis, J. Symbolic Logic 41(3) (1976), 663-671.

[51] R. Grossberg and S. Shelah, On the structure of $\operatorname{Ext}_{p}(G, \mathbf{Z})$, J. Algebra 121 (1989), 117-128. See also [52] below.

[52] R. Grossberg and S. Shelah, On cardinalities in quotients of inverse limits of groups, Math. Japonica 47 (1998), 189-197.

[53] A. Hajnal, True embedding partition relations, in: Finite and Infinite Combinatorics in Sets and Logic, Kluwer, 1993, 135-152.

[54] A. Hajnal and P. Hamburger, Halmazelmélet [Set Theory], Tankönyvkiaó Vállalat, Budapest, 1983 (in Hungarian).

[55] A. Hajnal, I. Juhász and Z. Szentmiklóssy, On the structure of CCC partial orders, Algebra Universalis, to appear.

[56] J. D. Hamkins, Every group has a terminating transfinite automorphism tower, Proc. Amer. Math. Soc. 126 (1998), 3223-3226.

[57] L. A. Harrington, M. D. Morley, A. Ščedrov and S. G. Simpson (eds.), Harvey Friedman's Research on the Foundations of Mathematics, Stud. Logic Found. Math. 117, North-Holland, 1985.

[58] W. Hodges, For singular $\lambda, \lambda$-free implies free, Algebra Universalis 12 (1981), 205-220.

[59] J. Ihoda [H. Judah] and S. Shelah, Souslin forcing, J. Symbolic Logic 53 (1988), 1188-1207. 
[60] T. Jech and S. Shelah, Possible pcf algebras, ibid. 61 (1996), 313-317; math.LO/ 9412208.

[61] H. Judah and S. Shelah, MA( $\sigma$-centered $)$ : Cohen reals, strong measure zero sets and strongly meager sets, Israel J. Math. 68 (1989), 1-17.

[62] H. Judah and S. Shelah, Baire Property and Axiom of Choice, Israel J. Math. 84 (1993), 435-450; math.LO/9211213.

[63] I. Juhász, Cardinal functions. II, in: Handbook of Set-Theoretic Topology, North-Holland, 1984, 63-109.

[64] —, Cardinal functions, in: Recent Progress in General Topology (Prague, 1991), North-Holland, Amsterdam, 1992, 417-441.

[65] I. Juhász and S. Shelah, How large can a hereditarily separable or hereditarily Lindelöf space be?, Israel J. Math. 53 (1986), 355-364.

[66] W. Just, A. R. D. Mathias, K. Prikry and P. Simon, On the existence of large p-ideals, J. Symbolic Logic 55 (1990), 457-465.

[67] W. Just, S. Shelah and S. Thomas, The automorphism tower problem III: Closed groups of uncountable degree, Adv. Math., accepted.

[68] A. S. Kechris and S. Solecki, Approximation of analytic by Borel sets and definable countable chain conditions, Israel J. Math. 89 (1995), 343-356.

[69] J. Ketonen, On the existence of P-points in the Stone-ČCh compactification of integers, Fund. Math. 92 (1976), 91-94.

[70] M. Kojman and S. Shelah, Non-existence of universal orders in many cardinals, J. Symbolic Logic 57 (1992), 875-891; math.LO/9209201.

[71] - - - The universality spectrum of stable unsuperstable theories, Ann. Pure Appl. Logic 58 (1992), 57-72; math.LO/9201253.

[72] P. Komjáth, On second-category sets, Proc. Amer. Math. Soc. 107 (1989), 653-654.

[73] D. W. Kueker, Countable approximations and Löwenheim-Skolem theorems, Ann. Math. Logic 11 (1977), 57-103.

[74] K. Kunen, Random and Cohen reals, in: Handbook of Set-Theoretic Topology, North-Holland, 1984, 887-911.

[75] -, Large homogeneous compact spaces, in: Open Problems in Topology, Elsevier, 1990, 261-270

[76] R. Laver, On the consistency of Borel's conjecture, Acta Math. 137 (1976), 151-169.

[77] M. Magidor and S. Shelah, Length of Boolean algebras and ultraproducts, Math. Japonica 48 (1998), 301-307; math.LO/9805145.

[78] A. R. D. Mathias, $0^{\#}$ and the p-point problem, in: Higher Set Theory (Oberwolfach, 1977), Lecture Notes in Math. 669, Springer, Berlin, 1978, 375-383.

[79] A. H. Mekler, A. Rosłanowski and S. Shelah, On the p-rank of Ext, Israel J. Math. 112 (1999), 327-356; math.LO/9806165

[80] A. H. Mekler and S. Shelah, Uniformization principles, J. Symbolic Logic 54 (1989), 441-459.

[81] —, -, Almost free algebras, Israel J. Math. 89 (1995), 237-259; math.LO/9408213.

[82] A. H. Mekler, S. Shelah and O. Spinas, The essentially free spectrum of a variety, Israel J. Math. 93 (1996), 1-8; math.LO/9411234.

[83] A. W. Miller, Arnie Miller's problem list, in: Set Theory of the Reals, Israel Math. Conf. Proc. 6, Bar-Ilan Univ., Ramat Gan, 1993, 645-654.

[84] D. Monk, Cardinal Invariants of Boolean Algebras, Progr. Math. 142, Birkhäuser, Basel, 1996. 
[85] J. Roitman, Adding a random or Cohen real: topological consequences and the effect on Martin's axiom, Fund. Math. 103 (1979), 47-60.

[86] A. Rosłanowski and S. Shelah, Forcing for $h L, h d$ and Depth, Colloq. Math., accepted; math.LO/9808104.

[87] - - - More on cardinal functions on Boolean algebras, Ann. Pure Appl. Logic, accepted; math.LO/9808056.

[88] -, -, Norms on possibilities III: strange subsets of the real line, in preparation.

[89] -, - Sweet $\&$ Sour and other flavours, preprint; math.LO/9909115.

[90] - - - Norms on possibilities II: more ccc ideals on $2^{\omega}$, J. Appl. Anal. 3 (1997), 103-127; math.LO/9703222.

[91] —, - Cardinal invariants of ultrapoducts of Boolean algebras, Fund. Math. 155 (1998), 101-151; math.LO/9703218.

[92] -, 一, Norms on possibilities I: forcing with trees and creatures, Mem. Amer. Math. Soc. 671 (1999); math.LO/9807172.

[93] M. Rubin and S. Shelah, Combinatorial problems on trees: partitions, $\Delta$-systems and large free subtrees, Ann. Pure Appl. Logic 33 (1987), 43-81.

[94] G. Sageev and S. Shelah, Noetherian ring with free additive groups, Abstracts Amer. Math. Soc. 7 (1986), 369.

[95] J. D. Sharp and S. Thomas, Uniformization problems and the cofinality of the infinite symmetric group, Notre Dame J. Formal Logic 35 (1994), 328-345.

[96] - - - Unbounded families and the cofinality of the infinite symmetric group, Arch. Math. Logic 34 (1995), 33-45.

[97] S. Shelah, Also quite large $\mathfrak{b} \subseteq \operatorname{pcf}(\mathfrak{a})$ behave nicely, math.LO/9906018.

[98] —, Analytical guide and corrections to [166], math.LO/9906022.

[99] -, Anti-homogeneous partitions of a topological space, Trans. Amer. Math. Soc., submitted; math.LO/9906025.

[100] -, Applications of PCF theory, J. Symbolic Logic, accepted; math.LO/9804155.

$[101]$ - Are $\mathfrak{a}$ and $\mathfrak{d}$ your cup of tea?, preprint.

[102] - Categoricity of an abstract elementary class in two successive cardinals, Israel J. Math., accepted; math.LO/9805146.

[103] - , Cellularity of free products of Boolean algebras (or topologies), this issue; math.LO/9508221.

[104] -, Constructing Boolean algebras for cardinal invariants, Algebra Univ., accepted; math.LO/9712286.

[105] - Covering of the null ideal may have countable cofinality, this issue; math.LO/ 9810181.

[106] —, Does a superatomic BA have an automorphism moving many atoms?, submitted to Proc. Hajnal Conference (New Brunswick, 1999).

[107] - Few non-minimal types and non-structure, in: Proc. 11 Internat. Congress of Logic, Methodology and Philosophy of Science (Krokow, 1999), Kluwer, to appear; math.LO/9906023.

[108] -, The future of set theory, in: Set Theory of the Reals, Israel Math. Conf. Proc. 6, Bar-Ilan Univ., Ramat Gan, 1993, 1-12.

[109] - Historic iteration with $\aleph_{\varepsilon}$-support, Arch. Math. Logic, accepted; math.LO/ 9607227

[110] - Iteration of $\lambda$-complete forcing notions not collapsing $\lambda^{+}$, in preparation; math.LO/9906024.

[111] -, Long iterations for the continuum

[112] - More constructions for Boolean algebras, Arch. Math. Logic, submitted; math.LO/9605235. 
[113] S. Shelah, More on Weak Diamond, East-West J. Math., accepted; math.LO/ 9807180 .

[114] —, Non-Structure Theory, volume accepted, Oxford Univ. Press.

[115] —, Non-elementary proper forcing notions, J. Appl. Anal., accepted; math.LO/ 9712283.

[116] -, Non-elementary proper forcing notions II, in preparation.

[117] -, Non-existence of universal members in classes of Abelian groups, J. Group Theory, accepted; math.LO/9808139.

[118] -, Not collapsing cardinals $\leq \kappa$ in $(<\kappa)$-support iterations, Israel J. Math., accepted; math.LO/9707225.

[119] - Not collapsing cardinals $\leq \kappa$ in $(<\kappa)$-support iterations II, ibid., submitted; math.LO/9808140.

[120] —, NNR revisited, J. Symbolic Logic, submitted.

[121] -, On ultraproducts and irr, preprint.

[122] -, On what I do not understand (and have something to say), model theory, Math. Japonica, accepted; math.LO/9910158.

[123] -, PCF and infinite free subsets, Arch. Math. Logic, accepted; math.LO/9807177.

[124] -, Choiceless polynomial time: Inability to express, in: Computer Science Logic, P. G. Clote and H. Schwichtenberg (eds.), Lecture Notes in Comput. Sci. 1862, Springer, 2000, 72-125; math.LO/9807179.

[125] —, Projective measurability does not imply projective Baire, in preparation.

[126] - Remarks on $\aleph_{1}-C W H$ not CWH first countable spaces, in: Set Theory (Boise, ID, 1992-1994), Contemp. Math. 192, Amer. Math. Soc., 1996, 103-145.

[127] - Special subsets of ${ }^{\operatorname{cf}(\mu)} \mu$, Boolean algebras and Maharam measure algebras, in: General Topology and its Applications-Proc. of Prague Topological Symposium 1996, accepted; math.LO/9804156.

[128] —, Strong covering without squares, this issue; math.LO/9604243.

[129] —, Strong dichotomy of cardinality, Results in Math., accepted; math.LO/ 9807183.

[130] —, The null ideal restricted to a non-null set may be saturated, math.LO/9705213.

[131] - Was Sierpiński right? IV, J. Symbolic Logic, accepted; math.LO/9712282.

[132] —, Notes on combinatorial set theory, Israel J. Math. 14 (1973), 262-277.

[133] -, A compactness theorem for singular cardinals, free algebras, Whitehead problem and transversals, ibid. 21 (1975), 319-349.

[134] —, Decomposing uncountable squares to countably many chains, J. Combin. Theory Ser. A 21 (1976), 110-114.

[135] - A combinatorial proof of the singular compactness theorem, mimeographed notes and lecture at a mini-conference, Berlin, August, 1977.

[136] -, Whitehead groups may be not free, even assuming CH. I, Israel J. Math. 28 (1977), 193-204

[137] —, Classification Theory and the Number of Nonisomorphic Models, Stud. Logic Found. Math. 92, North-Holland, Amsterdam, 1978.

[138] -, On successors of singular cardinals, in: Logic Colloquium '78 (Mons, 1978), Stud. Logic Found. Math. 97, North-Holland, Amsterdam, 1979, 357-380.

[139] —, Remarks on Boolean algebras, Algebra Universalis 11 (1980), 77-89.

[140] -, Canonization theorems and applications, J. Symbolic Logic 46 (1981), 345-353.

[141] —, On saturation for a predicate, Notre Dame J. Formal Logic 22 (1981), 239-248.

[142] - Proper Forcing, Lecture Notes in Math. 940, Springer, Berlin, 1982.

[143] -, Classification theory for nonelementary classes, I. The number of uncountable models of $\psi \in L_{\omega_{1}, \omega}$. Part B, Israel J. Math. 46 (1983), 241-273. 
[144] S. Shelah, Constructions of many complicated uncountable structures and Boolean algebras, ibid. 45 (1983), 100-146.

[145] - , Lifting problem of the measure algebra, ibid. 45 (1983), 90-96.

[146] —, Can you take Solovay's inaccessible away?, ibid. 48 (1984), 1-47.

[147] —, Diamonds, uniformization, J. Symbolic Logic 49 (1984), 1022-1033.

[148] - More on proper forcing, ibid. 49 (1984), 1034-1038.

[149] —, On logical sentences in PA, in: Logic Colloquium '82 (Florence, 1982), Stud. Logic Found. Math. 112, North-Holland, Amsterdam, 1984, 145-160.

[150] -, More on the weak diamond, Ann. Pure Appl. Logic 28 (1985), 315-318.

[151] - Remarks on the numbers of ideals of Boolean algebra and open sets of a topology, in: Around Classification Theory of Models, Lecture Notes in Math. 1182, Springer, Berlin, 1986, 151-187.

[152] —, Appendix: on stationary sets (in "Classification of nonelementary classes. II. Abstract elementary classes"), in: Classification Theory (Chicago, IL, 1985), Lecture Notes in Math. 1292, Springer, Berlin, 1987, 483-495.

[153] —, Can the fundamental (homotopy) group of a space be the rationals?, Proc. Amer. Math. Soc. 103 (1988), 627-632

[154] —, Primitive recursive bounds for van der Waerden numbers, J. Amer. Math. Soc. 1 (1988), 683-697.

[155] - Consistency of positive partition theorems for graphs and models, in: Set Theory and its Applications (Toronto, ON, 1987), Lecture Notes in Math. 1401, Springer, Berlin, 1989, 167-193.

[156] —, The number of pairwise non-elementarily-embeddable models, J. Symbolic Logic 54 (1989), 1431-1455

[157] —, Incompactness for chromatic numbers of graphs in: A Tribute to Paul Erdős, Cambridge Univ. Press, Cambridge, 1990, 361-371.

[158] —, Strong negative partition above the continuum, J. Symbolic Logic 55 (1990), 21-31.

[159] —, Reflecting stationary sets and successors of singular cardinals, Arch. Math. Logic 31 (1991), 25-53.

[160] -, Strong partition relations below the power set: consistency, was Sierpinski right, II?, in: Sets, Graphs, and Numbers (Budapest, 1991), Colloq. Math. Soc. János Bolyai 60, North-Holland, 1991, 637-638; math.LO/9201244.

[161] —, Cardinal arithmetic for skeptics, Bull. Amer. Math. Soc. 26 (1992), 197-210; math.LO/9201251.

[162] —, Vive la différence I: Nonisomorphism of ultrapowers of countable models, in: Set Theory of the Continuum, Math. Sci. Res. Inst. Publ. 26, Springer, 1992, 357-405; math.LO/9201245.

[163] —, Advances in cardinal arithmetic, in: Finite and Infinite Combinatorics in Sets and Logic, Kluwer, 1993, 355-383.

[164] —, More on cardinal arithmetic, Arch. Math. Logic 32 (1993), 399-428.

[165] - On $\mathrm{CH}+2^{\aleph_{1}} \rightarrow(\alpha)_{2}^{2}$ for $\alpha<\omega_{2}$, in: Logic Colloquium'90 (Helsinki, 1990), Lecture Notes Logic 2, Springer, 1993, 281-289; math.LO/9308212.

[166] -, Cardinal Arithmetic, Oxford Logic Guides 29, Oxford Univ. Press, 1994.

[167] —, Cardinalities of topologies with small base, Ann. Pure Appl. Logic 68 (1994), 95-113; math.LO/9403219.

[168] - How special are Cohen and random forcings, i.e. Boolean algebras of the family of subsets of reals modulo meagre or null, Israel J. Math. 88 (1994), 159-174; math.LO/9303208. 
[169] S. Shelah, Possibly every real function is continuous on a non-meagre set, Publ. Inst. Math. Beograd 57 (1995), 47-60; math.LO/9511220.

[170] - Further cardinal arithmetic, Israel J. Math. 95 (1996), 61-114; math.LO/ 9610226

[171] —, Large normal ideals concentrating on a fixed small cardinality, Arch. Math. Logic 35 (1996), 341-347; math.LO/9406219.

[172] - Colouring and non-productivity of $\aleph_{2}-c c .$, Ann. Pure Appl. Logic 84 (1997), 153-174; math.LO/9609218.

[173] —, Non existence of universals for classes like reduced torsion free abelian groups under non neccessarily pure embeddings, in: Advances in Algebra and Model Theory 9, Gordon and Breach, 1997, 229-286; math.LO/9609217.

[174] -, On $\sigma$-entangled linear orders, Fund. Math. 153 (1997), 199-275; math.LO/ 9609216

[175] — Set Theory without choice: not everything on cofinality is possible, Arch. Math. Logic 36 (1997), 81-125; math.LO/9512227.

[176] —, Proper and Improper Forcing, Springer, 1998.

[177] - , There may be no nowhere dense ultrafilter, in: Logic Colloquium Haifa'95, Lecture Notes Logic 11, Springer, 1998, 305-324; math.LO/9611221.

[178] - , The Generalized Continuum Hypothesis revisited, Israel J. Math. 116 (2000), 285-321; math.LO/9809200.

[179] - Note on $\omega$-nw-nep forcing notions, preprint.

[180] S. Shelah, J. Saxl and S. Thomas, Infinite products of finite simple groups, Trans. Amer. Math. Soc. 348 (1996), 4611-4641; math.IG/9605202

[181] S. Shelah and O. Spinas, On incomparability and related cardinal functions on ultraproducts of Boolean algebras, in preparation; math.LO/9903116.

[182] S. Shelah and L. Stanley, Consistency of partition relation for cardinal in $\left(\lambda, 2^{\lambda}\right)$, J. Symbolic Logic, submitted; math.LO/9710216.

[183] - - - A theorem and some consistency results in partition calculus, Ann. Pure Appl. Logic 36 (1987), 119-152.

[184] S. Shelah and J. Steprāns, Somewhere trivial automorphisms, J. London Math. Soc. 49 (1994), 569-580; math.LO/9308214.

[185] _ - - Martin's axiom is consistent with the existence of nowhere trivial automorphisms, Proc. Amer. Math. Soc., submitted.

[186] S. Shelah and S. Thomas, The cofinality spectrum of the infinite symmetric group, J. Symbolic Logic 62 (1997), 902-916; math.LO/9412230.

[187] S. Shelah and J. Zapletal, Canonical models for $\aleph_{1}$ combinatorics, Ann. Pure Appl. Logic 98 (1999), 217-259; math.LO/9806166.

[188] S. Solecki, Analytic ideals and their applications, Ann. Pure Appl. Logic, to appear.

[189] —, Analytic ideals, Bull. Symbolic Logic 2 (1996), 339-348.

[190] R. M. Solovay, Real valued measurable cardinals, in: Axiomatic Set Theory, Proc. Symposia Pure Math. 13, part 1, Providence, RI, Amer. Math. Soc. 1971, 397-428.

[191] S. G. Simpson (moderator), E-mail list for discussing foundations of mathematics, http://www.math.psu.edu/simpson/fom/.

[192] J. Steprāns and S. W. Watson, Homeomorphisms of manifolds with prescribed behaviour on large dense sets, Bull. London Math. Soc. 19 (1987), 305-310.

[193] S. Thomas, The cofinalities of the infinite-dimensional classical groups, J. Algebra 179 (1996), 704-719. 
[194] S. Todorčević, Partition Problems in Topology, Contemp. Math. 84, Amer. Math. Soc., Providence, RI, 1989.

[195] J. van Mill, An introduction to $\beta \omega$, in: Handbook of Set-Theoretic Topology, North-Holland, Amsterdam, 1984, 503-567.

[196] B. Veličković, Applications of the open coloring axiom, in: Set Theory of the Continuum (Berkeley, CA, 1989), Math. Sci. Res. Inst. Publ. 26, Springer, 1992, 137-154.

[197] S. W. Williams, Box products, in: Handbook of Set-Theoretic Topology, NorthHolland, Amsterdam, 1984, 169-200.

[198] H. Woodin, The Axiom of Determinacy, Forcing Axioms and the Nonstationary Ideal, de Gruyter Ser. Logic Appl. 1, de Gruyter, in press.

Institute of Mathematics

The Hebrew University of Jerusalem

91904 Jerusalem, Israel

E-mail: shelah@math.huji.ac.il
Department of Mathematics Rutgers University

New Brunswick, NJ 08854, U.S.A. URL: http://www.math.rutgers.edu/ shelah

Received 17 January 1999;

in revised form 9 August 1999 